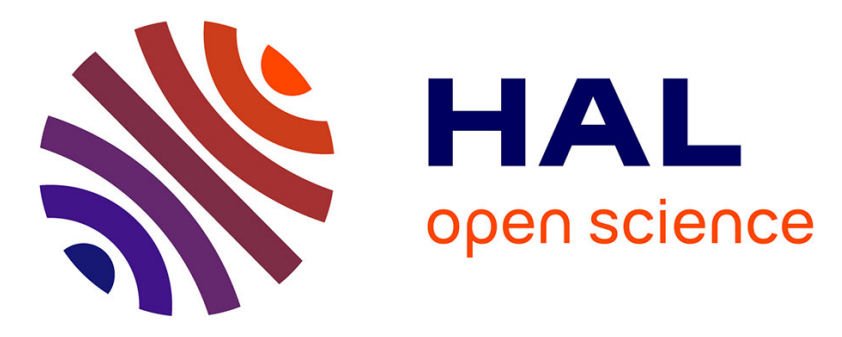

\title{
Modeling and computation of Bose-Einstein condensates: stationary states, nucleation, dynamics, stochasticity
}

Xavier Antoine, Romain Duboscq

\section{- To cite this version:}

Xavier Antoine, Romain Duboscq. Modeling and computation of Bose-Einstein condensates: stationary states, nucleation, dynamics, stochasticity. Lecture Notes in Mathematics, 2146, Springer, pp.49-145, 2015, Nonlinear Optical and Atomic Systems: at the Interface of Mathematics and Physics. hal-01094826

\section{HAL Id: hal-01094826 \\ https://hal.science/hal-01094826}

Submitted on 9 Feb 2016

HAL is a multi-disciplinary open access archive for the deposit and dissemination of scientific research documents, whether they are published or not. The documents may come from teaching and research institutions in France or abroad, or from public or private research centers.
L'archive ouverte pluridisciplinaire HAL, est destinée au dépôt et à la diffusion de documents scientifiques de niveau recherche, publiés ou non, émanant des établissements d'enseignement et de recherche français ou étrangers, des laboratoires publics ou privés. 


\title{
Modeling and computation of Bose-Einstein condensates: stationary states, nucleation, dynamics, stochasticity
}

\author{
Xavier ANTOINE and Romain DUBOSCQ
}

\begin{abstract}
The aim of this chapter is first to give an introduction to the derivation of the Gross-Pitaevskii Equations (GPEs) that arise in the modeling of Bose-Einstein Condensates (BECs). In particular, we describe some physical problems related to stationary states, dynamics, multi-components BECs and the possibility of handling stochastic effects into the equation. Next, we explain how to compute the stationary (and ground) states of the GPEs through the imaginary time method (also called Conjugate Normalized Gradient Flow) and finite difference or pseudo-spectral discretization techniques. Examples are provided by using GPELab which is a Matlab toolbox dedicated to the numerical solution of GPEs. Finally, we explain how to discretize correctly the time-dependent GPE so that the schemes are physically admissible. We again provide some examples by using GPELab. Furthermore, extensions of the discretization schemes to some classes of stochastic (in time) GPEs are described and analyzed.
\end{abstract}

\section{Modeling: Bose, Einstein, Gross \& Pitaevskii}

\subsection{From the theory to the realization of Bose-Einstein condensates}

The discovery of Bose-Einstein Condensates (BECs), from their theoretical prediction by Bose \& Einstein in 1925 to their first experimental realization in 1995 by Cornell and Wiemann, results from extraordinary scientific achievements that led to

Xavier ANTOINE

Université de Lorraine, Institut Elie Cartan de Lorraine, UMR 7502, Vandoeuvre-lès-Nancy, F54506, France \& Inria Nancy Grand-Est/IECL - ALICE, France. e-mail: xavier.antoinee univ-lorraine.fr

Romain DUBOSCQ

Université de Lorraine, Institut Elie Cartan de Lorraine, UMR 7502, Vandoeuvre-lès-Nancy, F54506, France. e-mail: romain.duboscq@univ-lorraine.fr 
the birth of condensed matter physics. The origin of the theory of BECs comes from an indian physicist, Satyendra Nath Bose, who proposed in 1924 a statistics for the photons that is different from the classical Maxwell-Boltzmann statistics. This latter allows to know the distribution of the particles velocity in an ideal gas with elastic shocks, corresponding to a classical description of matter. However, such statistics cannot be applied to microscopic particles where quantum effects must be included. An example is the Heisenberg uncertainty principle which states that both the position and velocity of a massive particle cannot be known simultaneously. Therefore, the introduction of a new statistical distribution of the particles in the phase space is required. In his works, Bose considers the photons which are particles that belong to the class of bosons (particles with an integer spin). Photons can occupy the same quantum state, implying that two photons with the same energy and position cannot be distinguished. Based on this property, Bose developed the foundations of the theory of quantum statistical mechanics. He sent his paper to Albert Einstein who submitted it for him to Zeitschrift für Physik [38] and who generalized this result to atoms [58]. In this work, Einstein predicts the existence of a new state of matter which is now better known as Bose-Einstein condensates. When a gas of dilute atoms is at a very low temperature (close to the absolute zero), there is a phase transition where a part of the gas condensates, which means that a large fraction of the atoms simultaneously occupy the lowest level quantum energy state, also called fundamental state. The critical temperature to observe the condensation phenomena is related to the property that the distance between the atoms is about one de Broglie wavelength $\lambda_{\text {de Broglie }}[52]$

$$
\lambda_{\text {de Broglie }}=\frac{h}{\left(2 \pi m k_{B} T\right)^{1 / 2}},
$$

where $h$ is the Planck constant, $m$ is the atomic mass, $k_{B}$ is the Boltzmann constant and $T$ is the temperature. When the characteristic distances of the system are about the same, quantum phenomena arise in the gas. A dimensional analysis argument [97] provides the formula

$$
T_{c}=3.3 \frac{\hbar^{2} n^{2 / 3}}{m k_{B}}
$$

to determine the critical temperature $T_{c}$, where $n$ is the number of particles per unit volume in the gas and $\hbar$ is the reduced Planck constant $\left(\hbar=\frac{h}{2 \pi}\right)$. At the time of these first predictions, experimentalists where not able to maintain the atoms in a gaseous state when cooling them, resulting in a transition to the solid state. In addition to the fact that extremely low temperatures had to be obtained, well-chosen candidates were required to experimentally observe the condensates.

In 1937, Kapitsa discovers the superfluidity phenomena that occurs in the helium gas [78]. Helium ${ }^{4} \mathrm{He}$ has the property to not solidify when it is cooled but to be in the liquid state even at very low temperatures. Kapitsa shows that a transition phase occurs in the helium fluid under $2.17 \mathrm{~K}$. Moreover, this new phase possesses amazing properties. For instance, there is almost no viscosity in the fluid. In 1938, London suggests that there is a connection between superfluidity in helium ${ }^{4} \mathrm{He}$ and BECs 
[88], the difference being that, in the case of the helium superfluid, only a small part of the atoms is at the fundamental state. The main reason is that strong interactions exist in the helium which is in a fluid state while BECs creation arises in ideal gazes with weak interactions. Nevertheless, the helium superfluid plays a key role in the development of some physical concepts that have next been applied to BECs. In 1949 , Onsager predicts the existence of quantum vortices in superfluids. His ideas have been further developed by Feynman [61, 62] in 1955. Quantum vortices are not an extension of classical vortices observed in a classical rotating fluid (like for example in water). For a superfluid, the velocity is given by the gradient of the phase function. Indeed, it is possible to describe a superfluid through a wavefunction

$$
\psi(t, \mathbf{x})=\sqrt{\rho(t, \mathbf{x})} e^{i S(t, \mathbf{x})},
$$

where $\rho(t, \mathbf{x})$ is the superfluid density and $S:=S(t, \mathbf{x})$ its phase, $\mathbf{x}:=(x, y, z) \in \mathbb{R}^{3}$ is a spatial point in the $\operatorname{system}\left(\mathbf{O}, \mathbf{e}_{x}, \mathbf{e}_{y}, \mathbf{e}_{z}\right)$ and $t>0$ is the time variable. The velocity of a superfluid is given by $\mathbf{v}(t, \mathbf{x})=\nabla S(t, \mathbf{x})$. Hence, a direct calculation shows that $\nabla \times \mathbf{v}(t, \mathbf{x})=\nabla \times \nabla S(t, \mathbf{x})=0$, where $S$ is smooth $(\mathbf{a} \times \mathbf{b}$ is the exterior product of two complex-valued vectors/operators $\mathbf{a}$ and $\mathbf{b}$ ). We can then deduce that the superfluid is irrotational where there is no singularity point, e.g. when the superfluid density is zero. These singularities create "holes" in the condensate that are called quantum vortices.

In 1959, Hecht suggests that the hydrogen atom with a polarized spin could be a suitable candidate to observe a condensate in the framework of weak interactions [72]. The interaction between two atoms of hydrogen with an aligned spin being weak, a cooling of the gas would not create a molecule nor a liquefaction. Hecht's idea is validated in practice in 1976 by Stwalley \& Nosanow [113] who confirmed the hypothesis of the weak interaction of hydrogen and hence started the race to the experimental realization of a hydrogen condensate. The first experiments used a magnetic field to cool the atoms. However, this technique was not robust enough since only a small part of the atoms was practically cooled. New cooling techniques were therefore necessary for confining the atoms. In 1987, a physics group from the Massachusetts Institute of Technology (MIT), supervised by Greytak \& Kleppner, published [74] a method where they first confined the hydrogen atoms by a magnetic trap and next cooled the gas to about $10^{-3} \mathrm{~K}$ by evaporation. Starting from a gas made of trapped atoms, the evaporation process consists in progressively letting the hottest atoms going out by diminishing the trap strength as illustrated on Figure 1 . We represent the trapping potential by a parabol and the atoms by small colored disks according to their temperature. During the cooling of the gas, a significant part of the atoms is lost. Therefore, it is necessary to start the process with a large enough quantity of atoms.

The realization of a condensate made of hydrogen atoms has been obtained in 1998 [63]. Meanwhile, the advances in terms of cooling by a laser, in particular for alkaline atoms, have finally led in 1995 the Boulder University group headed by Cornell \& Wieman to create the first Bose-Einstein condensate [9]. This BEC, made of rubidium atoms ${ }^{87} \mathrm{Rb}$, has been directly followed by a second realization 

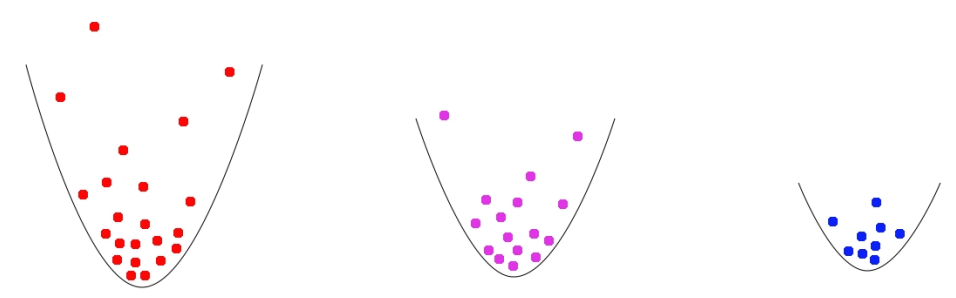

Fig. 1 Cooling of atoms by evaporation in a magnetic trap.

by Ketterle's team at the MIT by using sodium atoms ${ }^{23} \mathrm{Na}$ [51]. Cornell, Wieman $\&$ Ketterle have been awarded the Nobel prize in Physics in 2001 for their contributions on BECs. In parallel, a group from Rice University, supervised by Hulet, created a BEC with lithium atoms ${ }^{7} \mathrm{Li}[103]$. Since the lithium atoms are characterized by strong interactions, the condensate collapsed but Hulet was able to stabilize it through a quantum pressure technique. After these developments, other kinds of atoms were used to produce new BECs.

\subsection{Modeling Bose-Einstein condensates}

Various mathematical models can be used to describe BECs [15, 83, 104]. In this Section, we are most particularly interested in one of the most important models found in the Physics literature: the Gross-Pitaevskii Equation (GPE).

\subsubsection{From classical to quantum mechanics}

In quantum mechanics, the state of a system is described by a fundamental timedependent equation: the Schrödinger equation. This equation plays the role of the Euler-Lagrange or Hamilton equations used in classical mechanics. Let us assume that we have a physical system driven by the classical mechanics rules, for example a solid ball. The Lagrangian of a classical physical system [41] is given by

$$
\mathscr{L}=T_{\text {kin }}-V
$$

where $T_{\text {kin }}$ is the kinetic energy and $V$ is the potential energy of the system. For an object with mass $m$ which is assimilated to a point and subject to an exterior conservative force $\mathbf{F}(\mathbf{x})=-\nabla V(\mathbf{x})$ ( $\nabla$ is the usual gradient operator) at point $\mathbf{x}$, the kinetic and potential energies are respectively given by

$$
T_{\text {kin }}=\frac{1}{2} m|\dot{\mathbf{x}}(t)|^{2} \text { and } V=V(\mathbf{x}(t)),
$$


where $\dot{\mathbf{x}}(t)$ is the object velocity obtained by deriving its position $\mathbf{x}(t)$ with respect to the time variable $t$. Therefore, at a given time $t$, the Lagrangian depends on two variables that describe the configuration of the physical system: the speed and the position of the object. For a punctual object, one gets

$$
\mathscr{L}(\mathbf{x}, \dot{\mathbf{x}}, t)=\frac{1}{2} m|\dot{\mathbf{x}}(t)|^{2}-V(\mathbf{x}(t)) .
$$

The Euler-Lagrange equations characterize the dynamics of a classical system from its Lagrangian. They can be written as

$$
\frac{\partial \mathscr{L}}{\partial \mathbf{x}}(\mathbf{x}, \dot{\mathbf{x}}, t)-\frac{d}{d t} \frac{\partial \mathscr{L}}{\partial \dot{\mathbf{x}}}(\mathbf{x}, \dot{\mathbf{x}}, t)=0 .
$$

By applying this equation to the previous Lagrangian, we derive the fundamental equation of dynamics: $m \ddot{\mathbf{x}}(t)+\nabla V(\mathbf{x}(t))=0$, which provides the trajectory of the object.

The Hamilton's equations are a second approach to deduce the dynamics of a classical system [14]. We have already seen that the Lagrangian of a punctual object depends on both its position and velocity. It is possible to extend the expression of the Lagrangian by considering some generalized coordinates $\mathbf{q}$ and the associated generalized velocity $\dot{\mathbf{q}}$. The generalized coordinates must be chosen to uniquely define the configuration of the physical system. The Hamiltonian of the system is obtained by the following formula which corresponds to a Legendre transformation of the Lagrangian

$$
\mathscr{H}(\mathbf{q}, \mathbf{p}, t)=\mathbf{p} \cdot \mathbf{q}-\mathscr{L}(\mathbf{q}, \dot{\mathbf{q}}, t),
$$

$\mathbf{a} \cdot \mathbf{b}$ being the hermitian product between two complex-valued vector fields $\mathbf{a}$ and $\mathbf{b}$, the associated norm is $|\mathbf{a}|:=\sqrt{\mathbf{a} \cdot \mathbf{a}}$. In the previous equation, $\mathbf{p}$ denotes the generalized momentum such that

$$
\mathbf{p}=\frac{\partial \mathscr{L}}{\partial \dot{\mathbf{q}}}(\mathbf{q}, \dot{\mathbf{q}}, t)
$$

The Hamilton's equations are given by

$$
\dot{\mathbf{q}}=\frac{\partial \mathscr{H}}{\partial \mathbf{p}}(\mathbf{q}, \mathbf{p}, t), \quad \dot{\mathbf{p}}=-\frac{\partial \mathscr{H}}{\partial \mathbf{q}}(\mathbf{q}, \mathbf{p}, t) .
$$

By considering a particle subject to an exterior conservative force, we have seen that we obtain the Lagrangian (1). We determine the associated Hamiltonian by using the relations (2) and (3). Relation (3) allows us to identify the generalized impulsion of the particle: $\mathbf{p}=m \dot{\mathbf{q}}$. By using (2), we obtain the Hamiltonian of the particle

$$
\mathscr{H}(\mathbf{q}, \mathbf{p}, t)=\frac{1}{2 m}|\mathbf{p}|^{2}+V(\mathbf{q})=T_{\text {kin }}+V,
$$

where $\mathscr{H}$ is the sum of the kinetic energy $T_{\text {kin }}$ and the potential energy $V$ of the particle. The total energy $\mathscr{E}$ of the particle is given via the Hamiltonian 


$$
\mathscr{E}:=T_{\text {kin }}+V=\mathscr{H}(\mathbf{q}, \mathbf{p}, t) .
$$

This second approach is the one adopted to describe quantum particles. The main difference is related to the way the massive particles are considered. Indeed, the modeling of microscopic particles is realized through a wave function. The idea behind the oscillating nature of matter comes from some physical experiments where the duality wave-particles was observed [52, 73]. This duality is associated to the probabilistic character of quantum mechanics: this is not possible to know in a deterministic way the state of a quantum system. Following this point of view, a wave function $\psi$ is associated to a particle and leads to the probability to determine a particle at a given point of the space. The probability to find a particle in a volume $M$ at time $t$ is

$$
\mathbb{P}(\text { particle } \in M)=\int_{M}|\psi(t, \mathbf{x})|^{2} d \mathbf{x} \in[0,1],
$$

implying the so-called "mass conservation" property

$$
\mathbb{P}\left(\text { particle } \in \mathbb{R}^{3}\right)=\int_{\mathbb{R}^{3}}|\psi(t, \mathbf{x})|^{2} d \mathbf{x}=1 .
$$

This description of the particles is given by the de Broglie's relations [52] $\hat{\mathbf{p}}=\hbar \mathbf{k}$ and $\hat{\mathscr{E}}=\hbar \omega$, where $\hat{\mathbf{p}}$ is the impulsion of a particle and $\mathbf{k}$ its wave number. The total energy $\hat{\mathscr{E}}$ of a particle is the sum of its kinetic and potential energies, and $\omega$ its angular frequency. The relation expresses both the impulsion and the energy of the particle (assimilated to a wave function) under an operator form. Considering that a particle is given as the sum of monochromatic plane waves (by Fourier superposition)

$$
\psi(t, \mathbf{x})=\frac{1}{(2 \pi)^{4}} \int_{\mathbb{R} \times \mathbb{R}^{3}} \hat{\psi}(\omega, \mathbf{k}) e^{i(\mathbf{x} \cdot \mathbf{k}-\omega t)} d \mathbf{k} d \omega,
$$

the de Broglie's relations formally lead to

$$
\begin{aligned}
-i \hbar \nabla \psi(t, \mathbf{x}) & =\frac{1}{(2 \pi)^{4}} \int_{\mathbb{R} \times \mathbb{R}^{3}} \hat{\mathbf{p}} \hat{\psi}(\omega, \mathbf{k}) e^{i(\mathbf{x} \cdot \mathbf{k}-\omega t)} d \mathbf{k} d \omega, \\
i \hbar \partial_{t} \psi(t, \mathbf{x}) & =\frac{1}{(2 \pi)^{4}} \int_{\mathbb{R} \times \mathbb{R}^{3}} \hat{\mathscr{E}} \hat{\psi}(\omega, \mathbf{k}) e^{i(\mathbf{x} \cdot \mathbf{k}-\omega t)} d \mathbf{k} d \omega .
\end{aligned}
$$

This makes a parallel between the momentum operator $\hat{\mathbf{p}}$ and the operator $\nabla: \hat{\mathbf{p}} \sim$ $-i \hbar \nabla$, and between the energy operator $\hat{\mathscr{E}}$ and the partial derivative $\partial_{t}: \hat{\mathscr{E}} \sim i \hbar \partial_{t}$. By using relation (4) and the previous ones, we deduce the following evolution equation for the wave function with Hamiltonian $\mathscr{H}$

$$
i \hbar \partial_{t} \psi(t, \mathbf{x})=\mathscr{H}(\mathbf{x},-i \hbar \nabla, t) \psi(t, \mathbf{x}) .
$$

Hence, $\mathscr{H}$ is now considered as an operator. This famous equation has been derived by Schrödinger [106]. It provides the dynamics of the wave function associated to the particles. In the case of a particle subject to an exterior potential $V$, we have the following Hamiltonian 


$$
\mathscr{H}=\frac{1}{2 m}|\hat{\mathbf{p}}|^{2}+V(\mathbf{x}),
$$

which leads to the Schrödinger equation

$$
i \hbar \partial_{t} \psi(t, \mathbf{x})=-\frac{\hbar^{2}}{2 m} \Delta \psi(t, \mathbf{x})+V(\mathbf{x}) \psi(t, \mathbf{x}) .
$$

Let us now introduce a new energy $\mathscr{E}$ corresponding to the mean-value of the Hamiltonian

$$
\mathscr{E}(\psi)(t):=\int_{\mathbb{R}^{3}} \psi(t, \mathbf{x})^{*} \mathscr{H}(\mathbf{x},-i \hbar \nabla, t) \psi(t, \mathbf{x}) d \mathbf{x}
$$

where $\psi^{*}$ designates the complex conjugate function of $\psi$. We can also write this energy as

$$
\mathscr{E}=\langle\psi(t, \mathbf{x}), \mathscr{H}(\mathbf{x},-i \hbar \nabla, t) \psi(t, \mathbf{x})\rangle_{L_{\mathbf{x}}^{2}},
$$

where $\langle., .\rangle_{L_{\mathbf{x}}^{2}}$ is the hermitian inner product

$$
\forall(\psi, \phi) \in L_{\mathbf{x}}^{2} \times L_{\mathbf{x}}^{2},\langle\phi, \psi\rangle_{L_{\mathbf{x}}^{2}}:=\int_{\mathbb{R}^{3}} \phi(\mathbf{x})^{*} \psi(\mathbf{x}) d \mathbf{x}
$$

for square-integrable functions on $\mathbb{R}^{3}$

$$
L_{\mathbf{x}}^{2}=L^{2}\left(\mathbb{R}^{3}\right):=\left\{\phi: \mathbb{R}^{3} \rightarrow \mathbb{C} / \int_{\mathbb{R}^{3}}|\phi(\mathbf{x})|^{2} d \mathbf{x}<\infty\right\} .
$$

The associated norm in $L_{\mathbf{x}}^{2}$ is

$$
\forall \phi \in L_{\mathbf{x}}^{2},\|\phi\|_{L_{\mathbf{x}}^{2}}:=\langle\phi, \phi\rangle_{L_{\mathbf{x}}^{2}}^{1 / 2} .
$$

When the Hamiltonian is self-adjoint, i.e.,

$$
\forall \phi_{1}, \phi_{2} \in \mathscr{C}_{0}^{\infty}\left(\mathbb{R}^{3}\right),\left\langle\mathscr{H}(\mathbf{x},-i \hbar \nabla, t) \phi_{1}, \phi_{2}\right\rangle_{L_{\mathbf{x}}^{2}}=\left\langle\phi_{1}, \mathscr{H}(\mathbf{x},-i \hbar \nabla, t) \phi_{2}\right\rangle_{L_{\mathbf{x}}^{2}},
$$

and time homogeneous, i.e.

$$
\mathscr{H}(\mathbf{x},-i \hbar \nabla, t)=\mathscr{H}(\mathbf{x},-i \hbar \nabla)
$$

the energy $\mathscr{E}$ is conserved with respect to the time variable. Indeed, we have

$$
\begin{aligned}
\partial_{t} \mathscr{E}(\psi)= & \int_{\mathbb{R}^{3}}\left[\partial_{t} \psi(t, \mathbf{x})\right]^{*} \mathscr{H}(\mathbf{x},-i \hbar \nabla) \psi(t, \mathbf{x}) d \mathbf{x} \\
& +\int_{\mathbb{R}^{3}} \psi(t, \mathbf{x})^{*} \mathscr{H}(\mathbf{x},-i \hbar \nabla)\left[\partial_{t} \psi(t, \mathbf{x})\right] d \mathbf{x}
\end{aligned}
$$

Since $\psi$ satisfies the Schrödinger equation associated with $\mathscr{H}$, we deduce that 


$$
\begin{aligned}
\partial_{t} \mathscr{E}(\psi) & =\int_{\mathbb{R}^{3}}\left[-\frac{i}{\hbar} \mathscr{H}(\mathbf{x},-i \hbar \nabla) \psi(t, \mathbf{x})\right]^{*} \mathscr{H}(\mathbf{x},-i \hbar \nabla) \psi(t, \mathbf{x}) d \mathbf{x} \\
& +\int_{\mathbb{R}^{3}} \psi(t, \mathbf{x})^{*} \mathscr{H}(\mathbf{x},-i \hbar \nabla)\left[-\frac{i}{\hbar} \mathscr{H}(\mathbf{x},-i \hbar \nabla) \psi(t, \mathbf{x})\right] d \mathbf{x} .
\end{aligned}
$$

By using the property that the Schrödinger operator is self-adjoint, one gets

$$
\begin{aligned}
\partial_{t} \mathscr{E}(\psi) & =\frac{i}{\hbar} \int_{\mathbb{R}^{3}}[\mathscr{H}(\mathbf{x},-i \hbar \nabla) \psi(t, \mathbf{x})]^{*} \mathscr{H}(\mathbf{x},-i \hbar \nabla) \psi(t, \mathbf{x}) d \mathbf{x} \\
& -\frac{i}{\hbar} \int_{\mathbb{R}^{3}}[\mathscr{H}(\mathbf{x},-i \hbar \nabla) \psi(t, \mathbf{x})]^{*} \mathscr{H}(\mathbf{x},-i \hbar \nabla) \psi(t, \mathbf{x}) d \mathbf{x}=0 .
\end{aligned}
$$

In addition, we remark that

$$
\mathscr{H}(\mathbf{x},-i \hbar \nabla, t) \psi(t, \mathbf{x})=D_{\psi^{*}} \mathscr{E}(\psi)(t, \mathbf{x})
$$

where the derivative of the energy is defined as a functional derivative in $L_{\mathbf{x}}^{2}$ equipped with the hermitian inner product $\langle., .\rangle_{L_{\mathbf{x}}^{2}}$. More precisely, in equation (7), we differentiate $\mathscr{E}(\psi)$ with respect to $\psi^{*}$ by considering that $\psi$ and $\psi^{*}$ are independent: we identify $D_{\psi^{*}} \mathscr{E}(\psi)$ as satisfying

$$
\int_{\mathbb{R}^{3}} \phi^{*} D_{\psi^{*}} \mathscr{E}(\psi) d \mathbf{x}=\lim _{\eta \rightarrow 0} \frac{1}{\eta}\left(\int_{\mathbb{R}^{3}}(\psi+\eta \phi)^{*} \mathscr{H} \psi d \mathbf{x}-\int_{\mathbb{R}^{3}} \psi^{*} \mathscr{H} \psi d \mathbf{x}\right) .
$$

This energy allows us to come back to the Schrödinger equation associated with a system by an equation like (7).

The notion of wave function can be generalized to a system of $N_{\text {part }}$ particles by using the Hamiltonian: $\mathscr{H}=\mathscr{H}\left(\mathbf{x}_{1}, \ldots, \mathbf{x}_{N_{\text {part }}}, \mathbf{p}_{1}, \ldots, \mathbf{p}_{N_{\text {part }}}, t\right)$. For example, for $N_{\text {part }}$ noninteracting distinct particles under the action of an exterior potential $V$, the Hamiltonian is

$$
\mathscr{H}=\sum_{j=1}^{N_{\text {part }}}-\frac{\hbar^{2}}{2 m} \Delta_{\mathbf{x}_{j}}+V\left(\mathbf{x}_{j}\right),
$$

where $\mathbf{x}_{j}$ designates the position of the $j$-th particle, $j=1, \ldots, N_{\text {part }}$. We can then deduce the wave function $\psi=\psi\left(t, \mathbf{x}_{1}, \ldots, \mathbf{x}_{N_{\text {part }}}\right)$ for system 90 through a Schrödinger equation. One then gets

$$
\begin{aligned}
& \mathbb{P}\left(\text { particle } 1 \in M_{1}, \ldots, \text { particle } N_{\text {part }} \in M_{N_{\text {part }}}\right)= \\
& \qquad \int_{M_{1} \times \ldots \times M_{N_{\text {part }}}}\left|\psi\left(t, \mathbf{x}_{1}, \ldots, \mathbf{x}_{N_{\text {part }}}\right)\right|^{2} d \mathbf{x}_{1} \ldots d \mathbf{x}_{N_{\text {part }}},
\end{aligned}
$$

where $M_{j}$ is the $j$-th volume associated with the $j$-th particle, $1 \leq j \leq N_{\text {part }}$. 


\subsubsection{Application to Bose-Einstein condensates}

We propose here a construction that can be found in [97]. For a BEC, the set of condensed particles occupies the same quantum state, that is the ground state. The condensate is considered as a system of indistinguishable particles with the same wave function $\psi$. The condensate wave function writes down as

$$
\tilde{\psi}\left(t, \mathbf{x}_{1}, \mathbf{x}_{2}, \ldots, \mathbf{x}_{N_{\text {part }}}\right)=\prod_{j=1}^{N_{\text {part }}} \psi\left(t, \mathbf{x}_{j}\right)
$$

Furthermore, the condensate corresponds to a set of particles subject to an exterior potential $V$ and an interaction force $U_{\text {int }}$ between the particles that depends on the distance between two given particles. The Hamiltonian of the system is

$$
\mathscr{H}=\sum_{j=1}^{N_{\text {part }}}-\frac{\hbar^{2}}{2 m} \Delta_{\mathbf{x}_{j}}+V\left(\mathbf{x}_{j}\right)+\sum_{1 \leq k<j \leq N_{\text {part }}} U_{\text {int }}\left(\mathbf{x}_{j}-\mathbf{x}_{k}\right) .
$$

We obtain the energy $\mathscr{E}_{\text {sys }}$ of the system of particles at time $t$ by using formulation (10) and the mass conservation (5)

$$
\begin{aligned}
\mathscr{E}_{\text {sys }} & :=\int_{\left(\mathbb{R}^{3}\right)^{N_{\text {part }}}} \tilde{\psi}^{*}\left(t, \mathbf{x}_{1}, \ldots, \mathbf{x}_{N_{\text {part }}}\right) \mathscr{H} \tilde{\psi}\left(t, \mathbf{x}_{1}, \ldots, \mathbf{x}_{N_{\text {part }}}\right) d \mathbf{x}_{1} \ldots d \mathbf{x}_{N_{\text {part }}} \\
& =N_{\text {part }} \int_{\mathbb{R}^{3}}\left[\frac{\hbar^{2}}{2 m}|\nabla \psi(t, \mathbf{x})|^{2}+V(\mathbf{x})|\psi(t, \mathbf{x})|^{2}\right] d \mathbf{x} \\
& +N_{\text {part }} \frac{N_{\text {part }}-1}{2} \int_{\mathbb{R}^{3}} \int_{\mathbb{R}^{3}} U_{\text {int }}\left(\mathbf{x}^{\prime}-\mathbf{x}\right)\left|\psi\left(t, \mathbf{x}^{\prime}\right)\right|^{2} d \mathbf{x}^{\prime}|\psi(t, \mathbf{x})|^{2} d \mathbf{x} .
\end{aligned}
$$

Let us consider the variable change $\psi \rightarrow 1 / \sqrt{N_{\text {part }}} \psi$ and let us assume that the number of atoms is sufficiently large so that $\left(N_{\text {part }}-1\right) / N_{\text {part }} \approx 1$. This yields the normalized energy

$$
\begin{aligned}
& \mathscr{E}(\psi)= \\
& \int_{\mathbb{R}^{3}}\left[\frac{\hbar^{2}}{2 m}|\nabla \psi(t, \mathbf{x})|^{2}+\left(V(\mathbf{x})+\frac{1}{2} \int_{\mathbb{R}^{3}} U_{\text {int }}\left(\mathbf{x}^{\prime}-\mathbf{x}\right)\left|\psi\left(t, \mathbf{x}^{\prime}\right)\right|^{2} d \mathbf{x}^{\prime}\right)|\psi(t, \mathbf{x})|^{2}\right] d \mathbf{x} .
\end{aligned}
$$

To derive the Schrödinger equation that describes the evolution of the wave function $\psi$, we compute the functional derivative of the energy

$$
\begin{aligned}
\int_{\mathbb{R}^{3}} \phi^{*} D_{\psi^{*}} \mathscr{E}(\psi) d \mathbf{x}= & \int_{\mathbb{R}^{3}} \phi(\mathbf{x})^{*}\left(-\frac{\hbar^{2}}{2 m} \Delta+V(\mathbf{x})\right) \psi(t, \mathbf{x}) d \mathbf{x} \\
& +\frac{1}{2} \int_{\mathbb{R}^{3}} \phi(\mathbf{x})^{*}\left(\int_{\mathbb{R}^{3}} U_{\mathrm{int}}\left(\mathbf{x}^{\prime}-\mathbf{x}\right)\left|\psi\left(t, \mathbf{x}^{\prime}\right)\right|^{2} d \mathbf{x}^{\prime}\right) \psi(t, \mathbf{x}) d \mathbf{x} \\
& +\frac{1}{2} \int_{\mathbb{R}^{3}}\left(\int_{\mathbb{R}^{3}} U_{\mathrm{int}}\left(\mathbf{x}^{\prime}-\mathbf{x}\right) \psi\left(t, \mathbf{x}^{\prime}\right) \phi\left(\mathbf{x}^{\prime}\right)^{*} d \mathbf{x}^{\prime}\right)|\psi(t, \mathbf{x})|^{2} d \mathbf{x} .
\end{aligned}
$$


From the Fubini theorem and by assuming that the interaction potential $U_{\text {int }}$ is even $U_{\text {int }}\left(\mathbf{x}^{\prime}-\mathbf{x}\right)=U_{\text {int }}\left(\mathbf{x}-\mathbf{x}^{\prime}\right)$ for any points $\mathbf{x}$ and $\mathbf{x}^{\prime}$, we remark that

$$
\begin{aligned}
& \int_{\mathbb{R}^{3}}\left(\int_{\mathbb{R}^{3}} U_{\text {int }}\left(\mathbf{x}^{\prime}-\mathbf{x}\right) \psi\left(t, \mathbf{x}^{\prime}\right) \phi\left(\mathbf{x}^{\prime}\right)^{*} d \mathbf{x}^{\prime}\right)|\psi(t, \mathbf{x})|^{2} d \mathbf{x}= \\
& \int_{\mathbb{R}^{3}}\left(\int_{\mathbb{R}^{3}} U_{\text {int }}\left(\mathbf{x}-\mathbf{x}^{\prime}\right) \phi(\mathbf{x})^{*} \psi(t, \mathbf{x}) d \mathbf{x}\right)\left|\psi\left(t, \mathbf{x}^{\prime}\right)\right|^{2} d \mathbf{x}^{\prime} .
\end{aligned}
$$

Hence, one gets

$$
\begin{aligned}
\int_{\mathbb{R}^{3}} \phi^{*} D_{\psi^{*}} \mathscr{E}(\psi) d \mathbf{x}= & \int_{\mathbb{R}^{3}} \phi(\mathbf{x})^{*}\left(-\frac{\hbar^{2}}{2 m} \Delta+V(\mathbf{x})\right) \psi(t, \mathbf{x}) d \mathbf{x} \\
& +\int_{\mathbb{R}^{3}} \phi(\mathbf{x})^{*}\left(\int_{\mathbb{R}^{3}} U_{\mathrm{int}}\left(\mathbf{x}^{\prime}-\mathbf{x}\right)\left|\psi\left(t, \mathbf{x}^{\prime}\right)\right|^{2} d \mathbf{x}^{\prime}\right) \psi(t, \mathbf{x}) d \mathbf{x} .
\end{aligned}
$$

This leads to the Schrödinger equation satisfied by $\psi$

$$
i \hbar \partial_{t} \psi=D_{\psi^{*}} \mathscr{E}(\psi)=\left(-\frac{\hbar^{2}}{2 m} \Delta+V(\mathbf{x})+\int_{\mathbb{R}^{3}} U_{\mathrm{int}}\left(\mathbf{x}^{\prime}-\mathbf{x}\right)\left|\psi\left(t, \mathbf{x}^{\prime}\right)\right|^{2} d \mathbf{x}^{\prime}\right) \psi .
$$

Let us remark that the parity assumption of $U_{\text {int }}$ is not restrictive in practice because the interatomic interactions are symmetrical. Furthermore, this type of potential can describe a wide variety of interactions between the atoms. For instance, the Van der Waals interaction created by a dipole-dipole electric interaction between the atoms writes [97]

$$
U_{\mathrm{vdW}}\left(\left|\mathbf{x}-\mathbf{x}^{\prime}\right|\right)=-\frac{C_{6}}{\left|\mathbf{x}-\mathbf{x}^{\prime}\right|^{6}}
$$

From a mathematical point of view, we remark that the nonlocal interaction term is given by an integral operator. To avoid the problem of evaluating this class of interactions, physicists introduced the concept of effective interaction. By considering a system of two interacting particles with low energy, the interaction between the particles can be quantified by a constant $a$ that is usually called the "scattering length". This simplification leads to the computation of an effective interaction $U_{\text {eff }}$ between two particles that formally satisfies

$$
U_{0}:=\int_{\mathbb{R}^{d}} U_{\mathrm{eff}}\left(\mathbf{x}_{0}-\mathbf{x}\right) d \mathbf{x}=\frac{4 \pi \hbar^{2} a}{m},
$$

where $m$ is the mass of the particles, $\mathbf{x}_{0}$ is the reference particle position and $\mathbf{x}$ corresponds to the position of the other particle. Therefore, if we assume that the interatomic distance inside the condensate is sufficiently large compared to the scattering length $a$, the interaction between the particles can be replaced by a localized interaction which is proportional to $U_{0}$, that is: $U\left(\mathbf{x}_{0}-\mathbf{x}\right)=U_{0} \delta_{0}\left(\mathbf{x}_{0}-\mathbf{x}\right)$. We deduce a Schrödinger equation for the wave function $\psi$ as

$$
i \hbar \partial_{t} \psi(t, \mathbf{x})=\left(-\frac{\hbar^{2}}{2 m} \Delta+V(\mathbf{x})+\frac{4 \pi \hbar^{2} a}{m}|\psi(t, \mathbf{x})|^{2}\right) \psi(t, \mathbf{x}) .
$$


This equation has been obtained independently by Gross [71] and Pitaevskii [98] in 1961 and is called the Gross-Pitaevskii Equation (GPE). More recently, the equation was derived in more general frameworks [85, 86]. In the sequel, we consider this equation for modeling a BEC.

\subsection{Enrichment of the GPE: quantum vorticity, dipole-dipole interaction, multi-components, stochasticity}

\subsubsection{Rotating Bose-Einstein condensates and quantum vortices}

One of the most interesting characteristics of superfluids is their response to rotation. In a superfluid, the velocity of the fluid is given by the gradient of its wave function. As mentioned before, the fluid is irrotational everywhere except at the singularities called quantum vortices. Furthermore, another feature of superfluids is that there exists a characteristic velocity given by the spectrum of the excited states of the quantum system. Above this critical velocity, the system is excited. For example, an impurity moving in a superfluid will not cause any perturbation in the fluid unless its speed is above the critical velocity.

Since Bose-Einstein condensates are supposed to behave like superfluids, a lot of experiments were proposed to investigate properties like the existence of critical velocity or the nucleation of quantum vortices when a rotation is applied to the condensate. Two teams, one from the ENS Paris led by Dalibard [91, 92, 93] and a second one from the MIT and headed by Ketterle, have developed a method involving anisotropic harmonic potentials to stir the condensate and rotate it. They observed that there is no nucleation of vortices in the condensate under a certain rotation speed. The process of nucleation only begins when a certain rotational speed is obtained. In addition, the number of vortices is directly proportional to the rotation speed (see Figure 2).

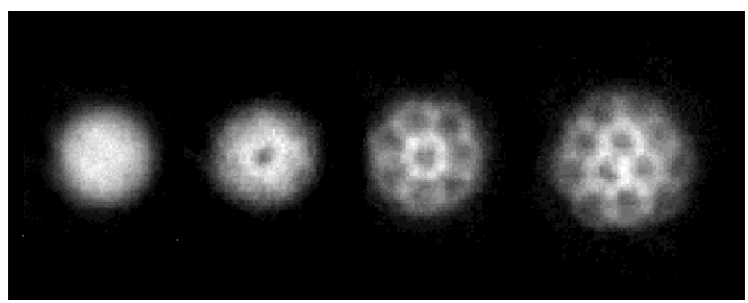

Fig. 2 Nucleation of vortices for an increasing rotation speed (from left to right). The experiments were done by a group led by Jean Dalibard in 2001 at the Kastler Brossel laboratory (ENS Paris, France).

For modeling a rotating BEC, we need to change from the reference frame of the laboratory to the rotating frame of the condensate. If the rotation axis is the $z$ - 
direction (i.e. $\Omega=(0,0, \Omega)$, where $\Omega$ is the rotation speed), this change of frame leads to the following transformation of variables

$$
\left\{\begin{array}{l}
x^{\prime}=\cos (\Omega t) x+\sin (\Omega t) y \\
y^{\prime}=-\sin (\Omega t) x+\cos (\Omega t) y
\end{array}\right.
$$

Therefore, to any vector $\mathbf{x}$ in the reference frame is associated a time dependent vector $\mathbf{x}^{\prime}(t)$ in the rotating frame. We remark that: $\dot{\mathbf{x}}(t)=\dot{\mathbf{x}}^{\prime}(t)+\Omega \times \mathbf{x}^{\prime}(t)$. We now consider a particle in rotation and subject to a potential $V$. The Lagrangian associated to this particle is

$$
\mathscr{L}(\mathbf{x}, \dot{\mathbf{x}}, t)=\frac{1}{2} m \dot{\mathbf{x}}(t)^{2}-V(\mathbf{x}(t)) .
$$

By using the change of variables, we deduce the Lagrangian in the rotating frame

$$
\mathscr{L}\left(\mathbf{x}^{\prime}, \dot{\mathbf{x}}^{\prime}, t\right)=\frac{1}{2} m\left(\dot{\mathbf{x}}^{\prime}(t)+\Omega \times \mathbf{x}^{\prime}(t)\right)^{2}-V\left(\mathbf{x}^{\prime}(t)\right)
$$

and the generalized momentum operator

$$
\mathbf{p}^{\prime}=\frac{\partial \mathscr{L}}{\partial \dot{\mathbf{x}}^{\prime}}=m\left(\dot{\mathbf{x}}^{\prime}+\Omega \times \mathbf{x}^{\prime}\right) .
$$

The Hamiltonian of a particle in the rotating frame is then

$$
\mathscr{H}\left(\mathbf{x}^{\prime}, \mathbf{p}^{\prime}, t\right)=\mathbf{p}^{\prime} \cdot \mathbf{x}^{\prime}-\mathscr{L}\left(\dot{\mathbf{x}}^{\prime}, \mathbf{x}^{\prime}, t\right)=\frac{\mathbf{p}^{\prime 2}}{2 m}-\left(\Omega \times \mathbf{x}^{\prime}\right) \cdot \mathbf{p}^{\prime}+V\left(\mathbf{x}^{\prime}\right) .
$$

Applying the same procedure for the Hamiltonian of a system of $N_{\text {part }}$ interacting particles under the action of a potential $V$, we obtain

$$
\mathscr{H}=\sum_{j=1}^{N_{\text {part }}}-\frac{\hbar^{2}}{2 m} \Delta_{\mathbf{x}_{j}}+i \hbar\left(\Omega \times \mathbf{x}_{j}\right) \cdot \nabla_{\mathbf{x}_{j}}+V\left(\mathbf{x}_{j}\right)+\sum_{1 \leq k<j \leq N_{\text {part }}} U_{\text {int }}\left(\mathbf{x}_{j}-\mathbf{x}_{k}\right) .
$$

Similarly, we deduce the GPE for a rotating BEC

$$
i \hbar \partial_{t} \psi(t, \mathbf{x})=\left(-\frac{\hbar^{2}}{2 m} \Delta-i \hbar \Omega \cdot(\mathbf{x} \times \nabla)+V(\mathbf{x})+\frac{4 \pi \hbar^{2} a}{m}|\psi(t, \mathbf{x})|^{2}\right) \psi(t, \mathbf{x}),
$$

since $(\Omega \times \mathbf{x}) \cdot \nabla=-\Omega \cdot(\mathbf{x} \times \nabla)$.

\subsubsection{BECs including dipolar interactions}

BECs were first obtained for alkali and hydrogen atoms. Since these two families of atoms have a weak magnetic moment, the magnetic dipole-dipole interaction can be neglected in the associated GPE. Latter, BECs made of chromium atoms ${ }^{52} \mathrm{Cr}$ were 
created, leading to a GPE where the dipole-dipole interactions must be included [69, 70]. As a consequence, an additional interaction term modeling the magnetic forces between the atoms has to be added to the Hamiltonian

$$
\mathscr{H}=\sum_{j=1}^{N_{\text {part }}}-\frac{\hbar^{2}}{2 m} \Delta_{\mathbf{x}_{j}}+V\left(\mathbf{x}_{j}\right)+\sum_{1 \leq k<j \leq N_{\text {part }}} U_{0} \delta_{0}\left(\mathbf{x}_{j}-\mathbf{x}_{k}\right)+U_{\text {dipole }}\left(\mathbf{x}_{j}-\mathbf{x}_{k}\right) .
$$

The notation $U_{\text {dipole }}$ corresponds to the magnetic dipole-dipole interaction given by

$U_{\text {dipole }}\left(\mathbf{x}_{j}-\mathbf{x}_{k}\right)=\frac{\mu_{0}}{4 \pi} \frac{\mu_{\mathrm{mag}, j}\left(\mathbf{x}_{j}\right) \cdot \mu_{\mathrm{mag}, k}\left(\mathbf{x}_{k}\right)-3\left(\mu_{\mathrm{mag}, j}\left(\mathbf{x}_{j}\right) \cdot \mathbf{u}_{j, k}\right)\left(\mu_{\mathrm{mag}, k}\left(\mathbf{x}_{k}\right) \cdot \mathbf{u}_{j, k}\right)}{\left|\mathbf{x}_{j}-\mathbf{x}_{k}\right|^{3}}$,

where $\mu_{\mathrm{mag}, j}$ (respectively $\mu_{\mathrm{mag}, k}$ ) is the magnetic momentum of the $j$-th atom (respectively $k$-th atom),

$$
\mathbf{u}_{j, k}=\frac{\mathbf{x}_{j}-\mathbf{x}_{k}}{\left|\mathbf{x}_{j}-\mathbf{x}_{k}\right|}
$$

and $\mu_{0}$ is the permeability of vacuum. We now assume that all the atoms are polarized by an external magnetic field in the $z$-direction, implying that $\mu_{\mathrm{mag}, j}=\mu_{\mathrm{mag}, k}=$ $\mu_{\text {mag }} \mathbf{e}_{z}$, where $\mu_{\text {mag }}$ is the amplitude of the magnetic momentum of chromium atoms. Thus, the magnetic dipole-dipole interaction is

$$
U_{\text {dipole }}\left(\mathbf{x}_{j}-\mathbf{x}_{k}\right)=\frac{\mu_{0} \mu_{\mathrm{mag}}^{2}}{4 \pi} \frac{1-3 \cos \left(\operatorname{angle}\left(\mathbf{x}_{j}-\mathbf{x}_{k}, \mathbf{e}_{z}\right)\right.}{\left|\mathbf{x}_{j}-\mathbf{x}_{k}\right|^{3}},
$$

where angle $\left(\mathbf{x}_{j}-\mathbf{x}_{k}, \mathbf{e}_{z}\right)$ is the angle between $\mathbf{x}_{j}-\mathbf{x}_{k}$ and $\mathbf{e}_{z}$. By using the Hamiltonian (12), we obtain the following GPE with a nonlocal interaction term

$$
\begin{aligned}
& i \hbar \partial_{t} \psi(t, \mathbf{x})= \\
& \left(-\frac{\hbar^{2}}{2 m} \Delta+V(\mathbf{x})+\left[\frac{4 \pi \hbar^{2} a}{m}|\psi(t, \mathbf{x})|^{2}+\frac{\mu_{0} \mu_{\mathrm{mag}}^{2}}{2 \pi} \int_{\mathbb{R}^{3}} U\left(\mathbf{x}-\mathbf{x}^{\prime}\right)\left|\psi\left(t, \mathbf{x}^{\prime}\right)\right|^{2} d \mathbf{x}^{\prime}\right]\right) \psi(t, \mathbf{x}),
\end{aligned}
$$

where

$$
U(\mathbf{x})=\frac{1-3 \cos \left(\operatorname{angle}\left(\mathbf{x}, \mathbf{e}_{z}\right)\right)}{|\mathbf{x}|^{3}} .
$$

\subsubsection{Origin of stochastic effects in BECs}

Some classes of GPEs include some random terms to describe the stochastic effects that may arise in BECs. For example, let us cite the modeling of random fluctuations in an optical trap [1, 2, 3, 67, 68, 105] or the consideration of the interactions between a cloud of non condensed atoms and the BEC [65, 66, 39, 110, 111]. This latter model involves a space and time stochastic process that describes the fluctuations of the phase and density of the condensate. Here, we focus on the first model and derive the associated stochastic GPE. 
In [105], the authors model randomness in the intensity of the optical trapping device used to confine the BEC. In the case of a magneto-optical trap, the laser beam used is slightly detuned to a frequency less than the resonant frequency of the atoms. When the laser beam is coupled to a spatially varying magnetic field which changes the resonant frequency of the atoms, a potential force is induced, then creating an atomic trapping device [94]. Using multiple laser beams leads to the potential

$$
V(t, \mathbf{x})=-\frac{1}{4} \alpha|E(t, \mathbf{x})|^{2},
$$

where $\alpha$ is the atomic polarizability and $E$ is the amplitude of the electric field generated by the laser beam. For a small detuning effect of the laser (less than 10\%), the atomic polarizability is given by the approximation: $\alpha=-\frac{\tau^{2}}{\hbar \Delta \omega}$, where $\tau$ is the transition dipole momentum and $\Delta \omega=\omega-\omega_{0}$ is the detuning parameter between the laser pulsation $\omega$ and the electronic transition pulsation $\omega_{0}$ of the atoms. For a gaussian laser beam, the intensity of the electric field created by the laser is

$$
|E(t, \mathbf{x})|^{2}=E_{0}(t)^{2} e^{-\frac{|\mathbf{x}|^{2}}{\ell^{2}}},
$$

where $\ell$ is the gaussian beam radius. If the size of the condensate is small compared to $\ell(|\mathbf{x}| \ll \ell)$, a Taylor's expansion gives the following approximation of the potential

$$
V(t, \mathbf{x})=-\frac{\alpha}{4}\left|E_{0}(t)\right|^{2}+\frac{\alpha}{4 \ell^{2}}\left|E_{0}(t)\right|^{2}|\mathbf{x}|^{2} .
$$

By a gauge transformation

$$
\psi(t, \mathbf{x}) \rightarrow \psi(t, \mathbf{x}) e^{\frac{i \alpha}{4 \hbar} \int_{0}^{t}\left|E_{0}(s)\right|^{2} d s},
$$

we eliminate in the GPE (11) the constant term that appears in the previous potential and obtain

$$
i \hbar \partial_{t} \psi(t, \mathbf{x})=\left(-\frac{\hbar^{2}}{2 m} \Delta+\frac{\alpha}{4 \ell^{2}}\left|E_{0}(t)\right|^{2}|\mathbf{x}|^{2}+\frac{4 \pi \hbar^{2} a}{m}|\psi(t, \mathbf{x})|^{2}\right) \psi(t, \mathbf{x}) .
$$

Finally, the fluctuations in the laser intensity are modeled by a random process $\dot{\xi}$ which determines the difference between the mean intensity $\left|E_{0}\right|^{2}$ and the intensity $\left|E_{0}(t)\right|^{2}$ at time $t$. If we set

$$
\dot{\xi}(t)=\frac{\left|E_{0}(t)\right|^{2}-\left|E_{0}\right|^{2}}{\left|E_{0}\right|^{2}}
$$

we deduce the following stochastic GPE

$$
i \hbar \partial_{t} \psi(t, \mathbf{x})=\left(-\frac{\hbar^{2}}{2 m} \Delta+\frac{\alpha}{4 \ell^{2}}\left|E_{0}\right|^{2}(1+\dot{\xi}(t))|\mathbf{x}|^{2}+\frac{4 \pi \hbar^{2} a}{m}|\psi(t, \mathbf{x})|^{2}\right) \psi(t, \mathbf{x}) .
$$


A process which is widely used by physicists is the brownian motion $\left(w_{t}\right)_{t \in \mathbb{R}^{+}}$. The associated noise (e.g. the time derivative of the process) is the so-called white noise $\left(\dot{w}_{t}\right)_{t \in \mathbb{R}^{+}}$, a real-valued centered gaussian process with covariance $\mathbb{E}\left[\dot{w}_{t} \dot{w}_{s}\right]=$ $\delta(t-s)$.

\subsubsection{Multi-components BECs}

In the derivation of the Gross-Pitaevskii model for BECs, we omitted the effect of the spin of the atoms. For each species of particles, there exists a principal quantum spin number $s$ that is either an half-integer for the fermions $(s=1 / 2,3 / 2, \ldots)$ or an integer for the bosons $(s=0,1,2, \ldots)$. The quantum spin number $S$ of a particle corresponds to a new degree of freedom $S \in\{-s,-(s-1), \ldots,(s-1), s\}$. For example, a fermion with principal quantum spin number $s=1 / 2$ can only have two possible spin numbers: $S=1 / 2$ or $S=-1 / 2$. Each value of $S$ corresponds to a quantum state of the particle. To describe the quantum system, we introduce a vector-valued wave function, where each component is associated to a value of $S$. This requires the extension of the GPE to a system of GPEs when $s \neq 0$. This situation corresponds to multi-components BECs.

Let us consider a mixture between two different species of atoms (denoted by type 1 and type 2), the extension to more components being direct. We suppose that each type of quantum system occupies the ground state. The first (respectively second) gas has $N_{\text {part, } 1}$ (respectively $N_{\text {part,2) }}$ ) atoms. Each component is described by a wave function, $\psi_{1}$ or $\psi_{2}$, according to the gas, that satisfies the mass conservation

$$
\int_{\mathbb{R}^{3}}\left|\psi_{\ell}(t, \mathbf{x})\right|^{2} d \mathbf{x}=N_{\text {part }, \ell}, \text { for } \ell=1,2
$$

The wave function of the BEC is

$$
\tilde{\psi}\left(t, \mathbf{x}_{1}^{1}, \ldots, \mathbf{x}_{N_{\text {part }, 1}^{1}}^{1}, \mathbf{x}_{1}^{2}, \ldots, \mathbf{x}_{N_{\text {part }, 2}^{2}}^{2}\right)=\prod_{j=1}^{N_{\text {part }, 1}} \psi_{1}\left(t, \mathbf{x}_{j}^{1}\right) \prod_{k=1}^{N_{\text {part }, 2}} \psi_{2}\left(t, \mathbf{x}_{k}^{2}\right),
$$

where $\mathbf{x}_{j}^{1}$ is the position of the $j$-th atom of type 1 and $\mathbf{x}_{k}^{2}$ is the position of the $k$ th atom of species 2. If we assume that the $\ell$-th component is subject to an external potential $V_{\ell}$, has a mass $m_{\ell}$ and that the components interact, we obtain the following Hamiltonian

$$
\begin{gathered}
\mathscr{H}=\sum_{j=1}^{N_{\text {part, } 1}}-\frac{\hbar^{2}}{2 m_{1}} \Delta_{\mathbf{x}_{j}^{1}}+V_{1}\left(\mathbf{x}_{j}^{1}\right)+\sum_{1 \leq j<l \leq N_{\text {part, }, 1}} U_{\text {int }, 1}\left(\mathbf{x}_{j}^{1}-\mathbf{x}_{l}^{1}\right)+\sum_{k=1}^{N_{\text {part }, 2}}-\frac{\hbar^{2}}{2 m_{2}} \Delta_{\mathbf{x}_{k}^{2}} \\
+V_{2}\left(\mathbf{x}_{j}^{2}\right)+\sum_{1 \leq k<m \leq N_{\text {part }, 2}} U_{\text {int }, 2}\left(\mathbf{x}_{k}^{2}-\mathbf{x}_{m}^{2}\right)+\sum_{j=1}^{N_{\text {part }, 1}} \sum_{k=1}^{N_{\text {part }, 2}} U_{\text {int }, 1,2}\left(\mathbf{x}_{j}^{1}-\mathbf{x}_{k}^{2}\right),
\end{gathered}
$$

where $U_{\mathrm{int}, \ell}$ corresponds to the interactions between the atoms of the type $\ell(=1,2)$ and $U_{\mathrm{int}, 1,2}$ describes the interatomic interaction. By symmetry, the interaction term 
$U_{\text {int, } 1,2}$ is even. We deduce the energy $\mathscr{E}_{\text {sys }}$ of the system by using (13)

$$
\begin{aligned}
\mathscr{E}_{\text {sys }}\left(\psi_{1}, \psi_{2}\right) & =N_{\text {part }, 1} \int_{\mathbb{R}^{3}}\left[\frac{\hbar^{2}}{2 m_{1}}\left|\nabla \psi_{1}(t, \mathbf{x})\right|^{2}+V_{1}(\mathbf{x})\left|\psi_{1}(t, \mathbf{x})\right|^{2}\right] d \mathbf{x} \\
& +N_{\text {part }, 1}\left(N_{\text {part }, 1}-1\right) / 2 \int_{\mathbb{R}^{3}} \int_{\mathbb{R}^{3}} U_{\text {int }, 1}\left(\mathbf{x}-\mathbf{x}^{\prime}\right)\left|\psi_{1}\left(t, \mathbf{x}^{\prime}\right)\right|^{2} d \mathbf{x}^{\prime}\left|\psi_{1}(t, \mathbf{x})\right|^{2} d \mathbf{x} \\
& +N_{\text {part }, 2} \int_{\mathbb{R}^{3}}\left[\frac{\hbar^{2}}{2 m_{2}}\left|\nabla \psi_{2}(t, \mathbf{x})\right|^{2}+V_{2}(\mathbf{x})\left|\psi_{2}(t, \mathbf{x})\right|^{2}\right] d \mathbf{x} \\
& +N_{\text {part }, 2}\left(N_{\text {part }, 2}-1\right) / 2 \int_{\mathbb{R}^{3}} \int_{\mathbb{R}^{3}} U_{\text {int }, 2}\left(\mathbf{x}-\mathbf{x}^{\prime}\right)\left|\psi_{2}\left(t, \mathbf{x}^{\prime}\right)\right|^{2} d \mathbf{x}^{\prime}\left|\psi_{2}(t, \mathbf{x})\right|^{2} d \mathbf{x} \\
& +N_{\text {part, }, 1} N_{\text {part }, 2} \int_{\mathbb{R}^{3}} \int_{\mathbb{R}^{3}} U_{\text {int, }, 2,2}\left(\mathbf{x}-\mathbf{x}^{\prime}\right)\left|\psi_{1}\left(t, \mathbf{x}^{\prime}\right)\right|^{2}\left|\psi_{2}(t, \mathbf{x})\right|^{2} d \mathbf{x}^{\prime} d \mathbf{x} .
\end{aligned}
$$

Let us consider the following changes of variables: $\psi_{\ell} \rightarrow 1 / \sqrt{N_{\text {part }, \ell}} \psi_{\ell}, \ell=1,2$. Moreover, we assume that $N_{\text {part }, \ell}$ is large enough to satisfy: $\left(N_{\text {part }, \ell}-1\right) / N_{\text {part }, \ell} \approx 1$, $\ell=1,2$. This leads to the normalized energy of the system

$$
\begin{aligned}
& \mathscr{E}\left(\psi_{1}, \psi_{2}\right)= \\
& \quad \int_{\mathbb{R}^{3}}\left[\frac{\hbar^{2}}{2 m_{1}}\left|\nabla \psi_{1}(t, \mathbf{x})\right|^{2}+\left(V_{1}(\mathbf{x})+\frac{1}{2} \int_{\mathbb{R}^{3}} U_{\mathrm{int}, 1}\left(\mathbf{x}-\mathbf{x}^{\prime}\right)\left|\psi_{1}\left(t, \mathbf{x}^{\prime}\right)\right|^{2} d \mathbf{x}^{\prime}\right)\left|\psi_{1}(t, \mathbf{x})\right|^{2}\right] d \mathbf{x} \\
& +\int_{\mathbb{R}^{3}}\left[\frac{\hbar^{2}}{2 m_{2}}\left|\nabla \psi_{2}(t, \mathbf{x})\right|^{2}+\left(V_{2}(\mathbf{x})+\frac{1}{2} \int_{\mathbb{R}^{3}} U_{\mathrm{int}, 2}\left(\mathbf{x}-\mathbf{x}^{\prime}\right)\left|\psi_{2}\left(t, \mathbf{x}^{\prime}\right)\right|^{2} d \mathbf{x}^{\prime}\right)\left|\psi_{2}(t, \mathbf{x})\right|^{2}\right] d \mathbf{x} \\
& +\int_{\mathbb{R}^{3}} \int_{\mathbb{R}^{3}} U_{\mathrm{int}, 1,2}\left(\mathbf{x}-\mathbf{x}^{\prime}\right)\left|\psi_{1}\left(t, \mathbf{x}^{\prime}\right)\right|^{2}\left|\psi_{2}(t, \mathbf{x})\right|^{2} d \mathbf{x}^{\prime} d \mathbf{x} .
\end{aligned}
$$

We obtain the Schrödinger equations that govern the evolution of $\psi_{\ell}, \ell=1,2$, by using the derivative of the energy with respect to $\psi_{1}$ and $\psi_{2}$. We have

$$
i \hbar \partial_{t} \psi_{\ell}(t, \mathbf{x})=D_{\psi_{\ell}^{*}} \mathscr{E}\left(\psi_{1}, \psi_{2}\right),
$$

for $\ell=1,2$, which is equivalent to the following system of equations

$$
\left\{\begin{array}{c}
i \hbar \partial_{t} \psi_{1}(t, \mathbf{x})=\left(-\frac{\hbar^{2}}{2 m_{1}} \Delta+V_{1}(\mathbf{x})+\int_{\mathbb{R}^{3}} U_{\text {int, } 1}\left(\mathbf{x}-\mathbf{x}^{\prime}\right)\left|\psi_{1}\left(t, \mathbf{x}^{\prime}\right)\right|^{2} d \mathbf{x}^{\prime}\right) \psi_{1}(t, \mathbf{x}) \\
+\int_{\mathbb{R}^{3}} U_{\text {int, } 1,2}\left(\mathbf{x}-\mathbf{x}^{\prime}\right)\left|\psi_{2}\left(t, \mathbf{x}^{\prime}\right)\right|^{2} d \mathbf{x}^{\prime} \psi_{1}(t, \mathbf{x}), \\
i \hbar \partial_{t} \psi_{2}(t, \mathbf{x})=\left(-\frac{\hbar^{2}}{2 m_{2}} \Delta+V_{2}(\mathbf{x})+\int_{\mathbb{R}^{3}} U_{\text {int, } 1}\left(\mathbf{x}-\mathbf{x}^{\prime}\right)\left|\psi_{2}\left(t, \mathbf{x}^{\prime}\right)\right|^{2} d \mathbf{x}^{\prime}\right) \psi_{2}(t, \mathbf{x}) \\
+\int_{\mathbb{R}^{3}} U_{\text {int, }, 1,2}\left(\mathbf{x}-\mathbf{x}^{\prime}\right)\left|\psi_{1}\left(t, \mathbf{x}^{\prime}\right)\right|^{2} d \mathbf{x}^{\prime} \psi_{2}(t, \mathbf{x}) .
\end{array}\right.
$$

Like for the one-component case, the interaction between the particles can be simplified by introducing an effective interaction

$$
U_{\mathrm{int}, \ell}\left(\mathbf{x}-\mathbf{x}^{\prime}\right)=\frac{4 \pi \hbar^{2} a_{\ell}}{m_{\ell}} \delta_{0}\left(\mathbf{x}-\mathbf{x}^{\prime}\right),
$$


where $a_{\ell}$ is the scattering length of the atoms of species $\ell(=1,2)$. The effective interatomic interaction is given by

$$
U_{\mathrm{int}, 1,2}\left(\mathbf{x}-\mathbf{x}^{\prime}\right)=\frac{4 \pi \hbar^{2} a_{1,2}}{m_{1,2}} \delta_{0}\left(\mathbf{x}-\mathbf{x}^{\prime}\right),
$$

where $a_{1,2}$ is the scattering length between an atom of type 1 and an atom of type 2 . The quantity $m_{1,2}$ is the reduced mass of a pair of atoms of types 1 and 2, i.e.

$$
m_{1,2}=\frac{m_{1} m_{2}}{m_{1}+m_{2}} .
$$

Finally, the evolution of a two-components BEC is modeled by the following system of GPEs

$$
\left\{\begin{aligned}
i \hbar \partial_{t} \psi_{1}(t, \mathbf{x})=\left(-\frac{\hbar^{2}}{2 m_{1}} \Delta+V_{1}(\mathbf{x})+\frac{4 \pi \hbar^{2} a_{1}}{m_{1}} \mid\right. & \left.\psi_{1}(t, \mathbf{x})\right|^{2} \\
& \left.+\frac{4 \pi \hbar^{2} a_{1,2}}{m_{1,2}}\left|\psi_{2}(t, \mathbf{x})\right|^{2}\right) \psi_{1}(t, \mathbf{x}), \\
i \hbar \partial_{t} \psi_{2}(t, \mathbf{x})=\left(-\frac{\hbar^{2}}{2 m_{2}} \Delta+V_{2}(\mathbf{x})+\frac{4 \pi \hbar^{2} a_{2}}{m_{2}} \mid\right. & \left.\psi_{2}(t, \mathbf{x})\right|^{2} \\
& \left.+\frac{4 \pi \hbar^{2} a_{1,2}}{m_{1,2}}\left|\psi_{1}(t, \mathbf{x})\right|^{2}\right) \psi_{2}(t, \mathbf{x}) .
\end{aligned}\right.
$$

\subsection{Stationary states}

In quantum mechanics, an excited state of a quantum system is a quantum state with an energy higher than the energy of the ground state (i.e. the quantum state with the lowest energy). Furthermore, the stationary states of a quantum system are the eigenfunctions of the Hamiltonian operator associated to the system. The eigenvalues for each stationary state are quantified energies related to the spectrum of the Hamiltonian operator. For a stationary state $\psi$, we have

$$
i \partial_{t} \psi=\mathscr{H} \psi=\mu \psi
$$

where $\mu$ is the eigenvalue linked to $\psi$. Therefore, the stationary state is searched as: $\psi(t, \mathbf{x})=\phi(\mathbf{x}) e^{-i \mu t}$, where $\phi$ is a time independent square-integrable function such that: $\|\phi\|_{L_{\mathrm{X}}^{2}}^{2}=1$. We directly compute an eigenvalue $\mu$, also called the chemical potential, by using the associated eigenfunction $\phi$ since

$$
\mu=\mu \int_{\mathbb{R}^{d}}|\phi|^{2} d \mathbf{x}=\int_{\mathbb{R}^{d}} \phi^{*} \mathscr{H} \phi d \mathbf{x} .
$$




\subsubsection{Critical points of the energy functional $\mathscr{E}$}

Stationary states are critical points of the energy functional. To prove this statement, we follow a proof similar to [97]. Let us consider a GPE for a rotating condensate

$i \hbar \partial_{t} \psi(t, \mathbf{x})=\left(-\frac{\hbar^{2}}{2 m} \Delta-i \hbar \Omega \cdot(\mathbf{x} \times \nabla)+V(\mathbf{x})+\left(N_{\text {part }}-1\right) \frac{4 \pi \hbar^{2} a}{m}|\psi(t, \mathbf{x})|^{2}\right) \psi(t, \mathbf{x})$.

We have seen that the energy associated to a given system, defined by (6), can be directly written through the Hamiltonian. For a nonlinear Hamiltonian, a corrective term must be added to fulfill (7)

$$
\begin{gathered}
\mathscr{E}(\psi)=\langle\psi(t, \mathbf{x}), \mathscr{H}(\mathbf{x},-i \hbar \nabla) \psi(t, \mathbf{x})\rangle_{L^{2}}-\int_{\mathbb{R}^{3}} \frac{1}{2} \frac{4 \pi \hbar^{2} a}{m}|\psi(t, \mathbf{x})|^{4} d \mathbf{x} \\
=\int_{\mathbb{R}^{3}}\left[\frac{\hbar^{2}}{2 m}|\nabla \psi(t, \mathbf{x})|^{2}+V(\mathbf{x})|\psi(t, \mathbf{x})|^{2}-\psi(t, \mathbf{x})^{*} i \hbar \Omega \cdot(\mathbf{x} \times \nabla) \psi(t, \mathbf{x})\right] d \mathbf{x} \\
+\int_{\mathbb{R}^{3}} \frac{1}{2} \frac{4 \pi \hbar^{2} a}{m}|\psi(t, \mathbf{x})|^{4} d \mathbf{x} .
\end{gathered}
$$

Let us set

$$
\mathscr{H}_{0}(\mathbf{x},-i \hbar \nabla)=-\frac{\hbar^{2}}{2 m} \Delta+V(\mathbf{x})-i \hbar \Omega \cdot(\mathbf{x} \times \nabla) .
$$

We remark that the Hamiltonian operator $\mathscr{H}_{0}$ is an hermitian operator in $L_{\mathbf{x}}^{2}$. Let us now compute the critical points of the energy functional (16) under the mass conservation constraint. To this end, we introduce a Lagrange multiplier $\lambda$ and solve

$$
D_{\psi^{*} \mathscr{E}}(\phi)+D_{\psi} \mathscr{E}(\phi)-\lambda\left[D_{\psi^{*}}\left(\|\psi\|_{L^{2}}\right)(\phi)+D_{\psi}\left(\|\psi\|_{L^{2}}\right)(\phi)\right]=0 .
$$

Since $\mathscr{H}_{0}$ is an hermitian operator, the functional derivatives are given by

$$
\begin{aligned}
& D_{\psi^{*}} \mathscr{E}(\phi)=D_{\psi} \mathscr{E}(\phi)=\mathscr{H}_{0} \phi+\frac{4 \pi \hbar^{2} a}{m}|\phi|^{2} \phi \\
& D_{\psi^{*}}\left(\|\psi\|_{L^{2}}\right)(\phi)=D_{\psi}\left(\|\psi\|_{L^{2}}\right)(\phi)=\phi(\mathbf{x}),
\end{aligned}
$$

leading a more explicit formulation of 17

$$
\mathscr{H}_{0} \psi+\frac{4 \pi \hbar^{2} a}{m}|\psi|^{2} \psi-\lambda \psi=0
$$

This finally means that the stationary states are the critical points of $\mathscr{E}$.

\subsubsection{Ansatz of the stationary states}

For a magneto-optical trap, the potential is 


$$
V(t, \mathbf{x})=-\frac{1}{4} \alpha|E(t, \mathbf{x})|^{2} .
$$

If we assume that the laser is gaussian, then it generates an electric field such that

$$
|E(t, \mathbf{x})|^{2}=E_{0}^{2} e^{-\frac{x^{2}}{\ell_{x}^{2}}-\frac{y^{2}}{\ell_{y}^{2}}-\frac{z^{2}}{\ell_{z}^{2}}}
$$

where $\ell_{x}, \ell_{y}$ and $\ell_{z}$ are the intensity radii of the beam in the $x$-, $y$-, and $z$-directions, respectively. Hence, if the characteristic length of the condensate is small compared to the lengths $\ell_{x, y, z}$ and if we use a gauge transformation, one gets the following GPE with harmonic trap

$$
i \hbar \partial_{t} \psi(t, \mathbf{x})=\left(-\frac{\hbar^{2}}{2 m} \Delta+\frac{\alpha}{4}\left|E_{0}\right|^{2}\left(\frac{x^{2}}{\ell_{x}^{2}}+\frac{y^{2}}{\ell_{y}^{2}}+\frac{z^{2}}{\ell_{z}^{2}}\right)+\frac{4 \pi \hbar^{2} a}{m}|\psi(t, \mathbf{x})|^{2}\right) \psi(t, \mathbf{x}) .
$$

For a noninteracting BEC (e.g. $a=0$ ), this system is a linear quantum harmonic oscillator. The ground state is then [97]

$$
\phi_{\mathrm{ho}}(\mathbf{x})=\frac{1}{\pi^{3 / 4}\left(a_{x} a_{y} a_{z}\right)^{1 / 2}} e^{-\frac{x^{2}}{2 a_{x}^{2}}-\frac{y^{2}}{2 a_{y}^{2}}-\frac{z^{2}}{2 a_{z}^{2}}},
$$

where

$$
a_{\{x, y, z\}}=\sqrt{\frac{\sqrt{2} \hbar l_{\{x, y, z\}}}{\left|E_{0}\right| \sqrt{\alpha m}}},
$$

with $a_{\{x, y, z\}}$ equal to $a_{x}, a_{y}$ or $a_{z}$ according to the subscript $x, y$ or $z$, respectively. The associated energy is

$$
\mathscr{E}_{\mathrm{osc}}:=\mathscr{E}\left(\phi_{\mathrm{ho}}\right)=\hbar\left|E_{0}\right| \sqrt{\frac{\alpha}{2 m}}\left(\frac{1}{\ell_{x}}+\frac{1}{\ell_{y}}+\frac{1}{\ell_{z}}\right) .
$$

If there are interactions inside the BEC (e.g. $a \neq 0$ ), we have

$$
\mathscr{E}\left(\phi_{\mathrm{ho}}\right)=\mathscr{E}_{\mathrm{osc}}+\frac{1}{2(2 \pi)^{3 / 2} a_{x} a_{y} a_{z}} \frac{4 \pi \hbar^{2} a}{m} .
$$

The gaussian function can still be considered as a suitable approximation of the exact ground state if the energy associated to the interaction term is small compared to the energy associated to the quantum harmonic oscillator. Thus, if $\ell_{x}=\ell_{y}=\ell_{z}$, the approximation of the ground state by a gaussian function is correct when $a_{x} \gg a$.

If the interaction energy is strong (e.g. $a \gg a_{x}$ ), this approximation is no longer valid. In this case, we consider the so-called Thomas-Fermi approximation [97, 99] which consists in neglecting the kinetic energy and keeping the potential and interaction energies, e.g. 
$\mathscr{E}_{\Delta}(\psi):=\int_{\mathbb{R}^{3}}|\nabla \psi|^{2} d \mathbf{x} \ll \mathscr{E}_{\mathrm{TF}}(\psi):=\int_{\mathbb{R}^{3}}\left[V(\mathbf{x})|\psi(t, \mathbf{x})|^{2}+\frac{1}{2} \frac{4 \pi \hbar^{2} a}{m}|\psi(t, \mathbf{x})|^{4}\right] d \mathbf{x}$.

The total energy is approximated by $\mathscr{E}(\psi) \approx \mathscr{E}_{\mathrm{TF}}(\psi)$. Let us set $U_{0}=\frac{4 \pi \hbar^{2} a}{m}$. The minimizer of $\mathscr{E}_{\mathrm{TF}}$ under the mass conservation constraint is computed by introducing a Lagrange multiplier $\mu_{\mathrm{TF}}$ and by solving

$$
\left(V+U_{0}\left|\phi_{\mathrm{TF}}\right|^{2}\right) \phi_{\mathrm{TF}}=\mu_{\mathrm{TF}} \phi_{\mathrm{TF}}
$$

Multiplying the previous equation by $\phi_{\mathrm{TF}}^{*}$ leads to

$$
\forall \mathbf{x} \in \operatorname{supp}\left(\phi_{\mathrm{TF}}\right), \quad\left|\phi_{\mathrm{TF}}(\mathbf{x})\right|^{2}=\frac{\mu_{\mathrm{TF}}-V(\mathbf{x})}{U_{0}} .
$$

Since $\left|\phi_{\mathrm{TF}}\right|^{2}>0$, it follows that

$$
\phi_{\mathrm{TF}}(\mathbf{x})= \begin{cases}\sqrt{\frac{\mu_{\mathrm{TF}}-V(\mathbf{x})}{U_{0}}}, & \text { for } \mu_{\mathrm{TF}}-V(\mathbf{x})>0 \\ 0 \quad & \text { for } \mu_{\mathrm{TF}}-V(\mathbf{x}) \leq 0\end{cases}
$$

The mass conservation gives the chemical potential $\mu_{\mathrm{TF}}$. For example, for a quadratic potential (with $\ell_{x}=\ell_{y}=\ell_{z}$ ), we have

$$
\mu_{\mathrm{TF}}=\frac{15^{2 / 5} \hbar^{2}}{2 m a_{x}}\left(\frac{a}{a_{x}}\right)^{2 / 5}
$$

\subsection{The rotating GPE with a quadratic potential: dimensionless form in 3d, 2d and 1d}

Let us consider the $3 \mathrm{~d}$ rotating GPE with a quadratic potential

$$
i \hbar \partial_{t} \psi=\left(-\frac{\hbar^{2}}{2 m} \Delta+\frac{\alpha}{4}\left|E_{0}\right|^{2}\left(\frac{x^{2}}{\ell_{x}^{2}}+\frac{y^{2}}{\ell_{y}^{2}}+\frac{z^{2}}{\ell_{z}^{2}}\right)-i \hbar \Omega \cdot(\mathbf{x} \times \nabla)+\frac{4 \pi \hbar^{2} a}{m}|\psi|^{2}\right) \psi
$$

where $\mathbf{x}=(x, y, z) \in \mathbb{R}^{3}$.

\subsubsection{Dimensionless form of the GPE}

Let us set

$$
\omega_{x}=\sqrt{\frac{\alpha}{2 m}} \frac{\left|E_{0}\right|}{\ell_{x}}, \omega_{y}=\sqrt{\frac{\alpha}{2 m}} \frac{\left|E_{0}\right|}{\ell_{y}}, \omega_{z}=\sqrt{\frac{\alpha}{2 m}} \frac{\left|E_{0}\right|}{\ell_{z}}, \quad U_{0}=\frac{4 \pi \hbar^{2} a}{m} .
$$

By using these new variables, the GPE writes down 


$$
i \hbar \partial_{t} \psi=\left(-\frac{\hbar^{2}}{2 m} \Delta+\frac{m}{2}\left(\omega_{x}^{2} x^{2}+\omega_{y}^{2} y^{2}+\omega_{z}^{2} z^{2}\right)-i \hbar \Omega \cdot(\mathbf{x} \times \nabla)+U_{0}|\psi|^{2}\right) \psi .
$$

Let us introduce the following changes of variables

$$
\begin{aligned}
& t \rightarrow \frac{t}{\omega_{m}}, \quad \omega_{m}=\min \left(\omega_{x}, \omega_{y}, \omega_{z}\right), \quad \mathbf{x} \rightarrow \mathbf{x} a_{0}, \\
& a_{0}=\sqrt{\frac{\hbar}{m \omega_{m}}}, \quad \psi \rightarrow \frac{\psi}{a_{0}^{3 / 2}}, \quad \Omega \rightarrow \Omega \omega_{m} .
\end{aligned}
$$

Then, we obtain the dimensionless GPE

$$
i \partial_{t} \psi=\left(-\frac{1}{2} \Delta+\frac{1}{2}\left(\gamma_{x}^{2} x^{2}+\gamma_{y}^{2} y^{2}+\gamma_{z}^{2} z^{2}\right)-i \Omega \cdot(\mathbf{x} \times \nabla)+\beta|\psi|^{2}\right) \psi,
$$

where $\gamma_{x}=\omega_{x} / \omega_{m}, \gamma_{y}=\omega_{y} / \omega_{m}, \gamma_{z}=\omega_{z} / \omega_{m}$ and $\beta=\frac{U_{0}}{a_{0}^{3} \hbar \omega_{m}}$.

\subsubsection{Dimension reductions}

Let us consider the dimensionless GPE

$$
i \partial_{t} \psi(t, \mathbf{x})=\left(-\frac{1}{2} \Delta+V(\mathbf{x})-i \Omega \cdot(\mathbf{x} \times \nabla)+\beta|\psi(t, \mathbf{x})|^{2}\right) \psi(t, \mathbf{x}),
$$

where $\Omega=(0,0, \Omega)$. We already know that a stationary state is a critical point of

$$
\mathscr{E}(\phi)=\int_{\mathbb{R}^{3}}\left(\frac{1}{2}|\nabla \phi(\mathbf{x})|^{2}+V(\mathbf{x})|\phi(\mathbf{x})|^{2}-\phi^{*}(\mathbf{x}) \Omega L_{z} \phi(t, \mathbf{x})+\frac{\beta}{2}|\phi(\mathbf{x})|^{4}\right) d \mathbf{x},
$$

with $L_{z}=-i\left(x \partial_{y}-y \partial_{x}\right)$. We assume that

$$
V(\mathbf{x})=\frac{1}{2}\left(\gamma_{x}^{2} x^{2}+\gamma_{y}^{2} y^{2}+\gamma_{z}^{2} z^{2}\right) .
$$

If $\gamma_{x} \approx \gamma_{y}$ and $\gamma_{z} \gg \gamma_{x}$, the condensate has a stationary state that expands in the $x$ - and $y$-directions but is confined along the $z$-axis (disc-shaped condensate). Indeed, the energy associated to the potential operator in the $z$-direction is large compared to the energies in the $x$ - and $y$-directions. Most particularly, an excitation of the condensate generates less dynamics in the $z$ - than in the $x$ - and $y$-directions [30]. Therefore, the dynamical solution is written as [76, 84]: $\psi(t, \mathbf{x})=\psi_{2}(t, x, y) \psi_{3}(z)$, where

$$
\psi_{3}(z)=\left(\int_{\mathbb{R}^{2}}\left|\psi_{0}(x, y, z)\right|^{2} d x d y\right)^{1 / 2}
$$

setting $\psi_{0}$ as the $3 \mathrm{~d}$ stationary state. Since $\psi_{0}$ is normalized, we have

$$
\int_{\mathbb{R}}\left|\psi_{3}(z)\right|^{2} d z=1
$$


Injecting $\psi$ in 20 , we obtain

$$
\begin{aligned}
i \psi_{3}(z) \partial_{t} \psi_{2}(t, x, y)= & -\frac{1}{2} \psi_{3}(z) \Delta \psi_{2}(t, x, y)-\frac{1}{2} \psi_{2}(t, x, y) \partial_{z}^{2} \psi_{3}(z) \\
& +\frac{1}{2}\left(\gamma_{x}^{2} x^{2}+\gamma_{y}^{2} y^{2}\right) \psi_{2}(t, x, y) \psi_{3}(z)+\frac{1}{2} \gamma_{z}^{2} z^{2} \psi_{2}(t, x, y) \psi_{3}(z) \\
& -\psi_{3}(z) \Omega L_{z} \psi_{2}(t, x, y)+\beta\left|\psi_{2}(t, x, y)\right|^{2}\left|\psi_{3}(z)\right|^{2} \psi_{2}(t, x, y) \psi_{3}(z) .
\end{aligned}
$$

Multiplying by $\psi_{3}^{*}$ and integrating on the whole space with respect to $z$ leads to

$$
i \partial_{t} \psi_{2}(t, x, y)=\left(-\frac{1}{2} \Delta+\frac{1}{2}\left(\gamma_{x}^{2} x^{2}+\gamma_{y}^{2} y^{2}\right)-\Omega L_{z}+\frac{1}{2} \sigma+\kappa_{2}\left|\psi_{2}(t, x, y)\right|^{2}\right) \psi_{2}(t, x, y),
$$

where

$$
\sigma=\int_{\mathbb{R}}\left(\gamma_{z}^{2} z^{2}\left|\psi_{3}(z)\right|^{2}+\left|\partial_{z} \psi_{3}(z)\right|^{2}\right) d z, \quad \kappa_{2}=\int_{\mathbb{R}} \beta\left|\psi_{3}(z)\right|^{4} d z .
$$

By using the gauge transformation $\psi_{2}(t, x, y) \rightarrow \psi(t, x, y) e^{-\frac{i \sigma}{2} t}$, one gets the twodimensional rotating GPE

$$
i \partial_{t} \psi(t, x, y)=\left(-\frac{1}{2} \Delta+\frac{1}{2}\left(\gamma_{x}^{2} x^{2}+\gamma_{y}^{2} y^{2}\right)-\Omega L_{z}+\kappa_{2}|\psi(t, x, y)|^{2}\right) \psi(t, x, y) .
$$

Let us now assume that the BEC is nonrotating and that $\gamma_{y, z} \gg \gamma_{x}$. Similar arguments to the previous ones [30, 84, 76] show that $\psi(t, \mathbf{x})=\psi_{1}(t, x) \psi_{2,3}(y, z)$, where

$$
\psi_{2,3}(y, z)=\left(\int_{\mathbb{R}}\left|\psi_{0}(x, y, z)\right|^{2} d x\right)^{1 / 2}
$$

Here, we assume that the condensate is cigar-shaped. Similarly to the $2 \mathrm{~d}$ reduction, we obtain the following one-dimensional GPE

$$
i \partial_{t} \psi(t, x)=\left(-\frac{1}{2} \partial_{x}^{2}+\frac{1}{2} \gamma_{x}^{2} x^{2}+\kappa_{1}|\psi(t, x)|^{2}\right) \psi(t, x),
$$

where

$$
\kappa_{1}=\int_{\mathbb{R}^{2}} \beta\left|\psi_{2,3}(y, z)\right|^{4} d y d z .
$$

Finally, a general form of the rotating GPE in dimension $d(=1,2,3)$ is

$$
i \partial_{t} \psi(t, \mathbf{x})=\left(-\frac{1}{2} \Delta+V_{d}(\mathbf{x})-\Omega_{d} L_{z}+\kappa_{d}|\psi(t, \mathbf{x})|^{2}\right) \psi(t, \mathbf{x}),
$$

where $\Omega_{2,3}=\Omega, \Omega_{1}=0$ (no rotation), 


$$
\kappa_{d}= \begin{cases}\int_{\mathbb{R}^{2}} \beta\left|\psi_{2,3}(y, z)\right|^{4} d y d z, & \text { for } d=1 \\ \int_{\mathbb{R}} \beta\left|\psi_{3}(z)\right|^{4} d z, & \text { for } d=2 \\ \beta, & \text { for } d=3\end{cases}
$$

and

$$
V_{d}(\mathbf{x})= \begin{cases}1 / 2 \gamma_{x}^{2} x^{2}, & \text { for } d=1, \\ 1 / 2\left(\gamma_{x}^{2} x^{2}+\gamma_{y}^{2} y^{2}\right), & \text { for } d=2, \\ 1 / 2\left(\gamma_{x}^{2} x^{2}+\gamma_{y}^{2} y^{2}+\gamma_{z}^{2} z^{2}\right), & \text { for } d=3\end{cases}
$$

\section{Computation: stationary states and nucleation}

\subsection{Stationary states formulation: solving a minimization problem for the energy functional or a nonlinear eigenvalue problem (under constraint)?}

The critical points of the energy functional associated to a GPE-like system are in fact the stationary states (see Section 1.4. page 177. An impressive number of publications has been devoted to this topic over the last years in the condensed matter physics literature (see for example [42, 48, 82, 109]). Indeed, stationary states correspond to (meta)stable states of the condensate. As seen in the first Section, the practical realization of a BEC requires a sophisticated experimental system that is only owned by a few laboratories worldwide. More generally, reaching a temperature to condensate the atomic gas is very challenging. In addition, imaging a condensate is a difficult task due to its small size. A widely used technique consists in letting the condensate expands during a short time scale and then imaging it when its size is large enough [59]. Let us remark that imaging a condensate destroys it immediately. As a consequence, some physical phenomenae are extremely difficult to observe in a BEC on a larger time scale [40, 60, 100]. Therefore, numerical simulations are helpful [33, 79, 108] to provide a complete visualization of a BEC and to compute some of its features (e.g. phase structure) in some various and complex situations (e.g. multi-components, different potentials, nonlinear long-range interactions). The limitations are essentially due to the model that is chosen.

Let us consider the model problem of a GPE with a nonlinearity defined by a function $f$ and with a rotation term

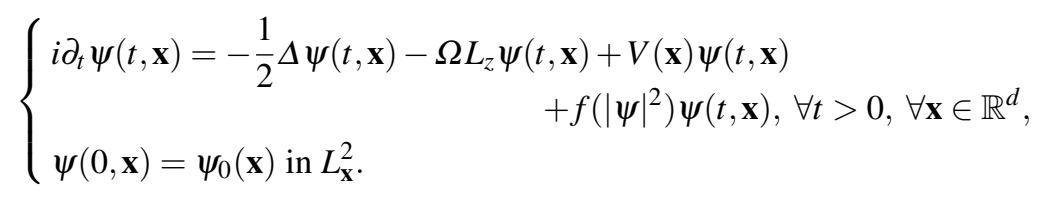


Function $V$ which acts from $\mathbb{R}_{\mathbf{x}}^{d}$ onto $\mathbb{R}^{+}$corresponds to a (confining) potential. Function $f$ can be a real-valued smooth function like for the standard case $f\left(|\psi|^{2}\right)=\beta|\psi|^{2 \sigma}$, with $\beta \in \mathbb{R}$ and $\sigma>0$. In practice, many other situations exist. As seen before (Section 1.3.2, page 12), $f$ is not necessarily a function but can also be an integro-differential operator like for dipole-dipole magnetic interactions. The parameter $\Omega \in \mathbb{R}$ is the rotation speed. The rotation operator $L_{z}$ is given by: $L_{z}=-i\left(x \partial_{y}-y \partial_{x}\right)$. To fix the ideas, let us remark that we may choose a transverse rotation which is written via the operator $L_{z}$. For $d=1$, there is no rotation $(\Omega=0)$.

The computation of the stationary states can be done via the minimization of the energy under constraint. For 21, the energy is given by

$$
\mathscr{E}_{\Omega, F}(\psi):=\int_{\mathbb{R}^{d}}\left(\frac{1}{2}|\nabla \psi|^{2}+V(\mathbf{x})|\psi|^{2}-\Omega \psi^{*} L_{z} \psi+F\left(|\psi|^{2}\right)\right) d \mathbf{x},
$$

where $F$ is the primitive function of $f$

$$
\forall r \in\left[0, \infty\left[, \quad F(r):=\int_{0}^{r} f(q) d q .\right.\right.
$$

Hence, the minimization problem consists in computing a function $\phi \in L_{\mathbf{x}}^{2}$ such that

$$
\mathscr{E}_{\Omega, F}(\phi)=\min _{\|\psi\|_{L_{\mathbf{X}}^{2}}=1} \mathscr{E}_{\Omega, F}(\psi) .
$$

From a numerical point of view, this implies that a strategy based on numerical nonlinear optimization techniques under constraints can be used to obtain the stationary states. A second approach is related to the property (see Section 1.4.1, page 18) that the problem can also be formulated as the nonlinear eigenvalue problem: find an eigenfunction $\phi \in L_{\mathbf{x}}^{2}$ and an eigenvalue $\mu \in \mathbb{R}$ such that

$$
\mu \phi=-\frac{1}{2} \Delta \phi-\Omega L_{z} \phi+V(\mathbf{x}) \phi+f\left(|\phi|^{2}\right) \phi,
$$

under the $L_{\mathbf{x}}^{2}$-normalization constraint for $\phi$. Concerning the nonlinear eigenvalue solvers, we refer for example to [55] for an application in the framework of GPEs.

Here, we essentially develop a method that is embedded in the class of the minimization methods. This approach is called Conjugate Normalized Gradient Flow (CNGF) and corresponds to the well-known imaginary time method in physics [4, 22, 34, 46, 47, 57, 64]. Let us however remark that other minimization methods can be used [30, 45, 50, 55]. The CNGF method consists in building a minimizing sequence of the energy functional $\mathscr{E}_{\Omega, F}$ given by $(22)$. To this end, we consider a time discretization $\left(t_{n}\right)_{n \in \mathbb{N}}$, with $t_{0}=0$, and we define the local time step: $\delta t_{n}=t_{n+1}-t_{n}, \forall n \in \mathbb{N}$. The CNGF method is given by the algorithm: compute the sequence of iterates $\left(\phi\left(\mathbf{x}, t_{n}\right)\right)_{n \in \mathbb{N}}$ defined by 


$$
\left\{\begin{aligned}
& \partial_{t} \phi(\mathbf{x}, t)=-D_{\phi^{*}} \mathscr{E}_{\Omega, F}(\phi)= \frac{1}{2} \Delta \phi(\mathbf{x}, t)+\Omega L_{z} \phi(\mathbf{x}, t)-V(\mathbf{x}) \phi(\mathbf{x}, t) \\
&-f\left(|\phi|^{2}\right) \phi(\mathbf{x}, t), \quad \forall t \in\left[t_{n}, t_{n+1}\left[, \forall \mathbf{x} \in \mathbb{R}^{d},\right.\right. \\
& \phi\left(\mathbf{x}, t_{n+1}\right):=\phi\left(\mathbf{x}, t_{n+1}^{+}\right)= \frac{\phi\left(\mathbf{x}, t_{n+1}^{-}\right)}{\left\|\phi\left(\mathbf{x}, t_{n+1}^{-}\right)\right\|_{L_{\mathbf{x}}^{2}}}, \\
& \phi(\mathbf{x}, 0)=\phi_{0}(\mathbf{x}) \in L_{\mathbf{x}}^{2}, \text { with }\left\|\phi_{0}\right\|_{L_{\mathbf{x}}^{2}}=1 .
\end{aligned}\right.
$$

In the above equations, we designate by $g\left(t_{n+1}^{+}\right)$(respectively $g\left(t_{n+1}^{-}\right)$) the limit from the right (respectively from the left) of a function $g$. The discrete times $t_{n}$ parametrize the sequence. This explains why we use the "inverse" notation $\phi(\mathbf{x}, t)$ instead of $\phi(t, \mathbf{x})$. Correctly choosing the initial data $\phi_{0}$ in the iterative algorithm is important to ensure the convergence. In practice, as we will see later in Section 2.3 (page 37), a suitable choice consists in considering initial data built as Ansatz of the underlying equation (GPE) with respect to a given asymptotic regime.

The CNGF method conserves the $L_{\mathbf{x}}^{2}$-norm of the solution [22]. Moreover, Bao $\& \mathrm{Du}[22]$ proved that the algorithm (25) produces a sequence that minimizes the energy in the linear case (i.e. $f\left(|\phi|^{2}\right)=0$ ) for a positive potential (i.e. $V(\mathbf{x}) \geq 0$ ). Hence, under these assumptions, we prove that

$$
\lim _{t \rightarrow \infty} \phi(\mathbf{x}, t)=\phi_{g}(\mathbf{x}),
$$

where $\phi_{g}$ is a stationary state. Practically, the long time computation 26 is fixed according to a stopping criterion that we will precise later.

\subsection{Time and space discretizations of system (25)}

In this Section, we consider several time and space discretization schemes for the system (25). The Partial Differential Equation that we want to solve is similar to a heat equation (and not a Schrödinger equation) in imaginary time. At first glance, one may think that using a standard method adapted to this class of equations would lead to an admissible scheme. Nevertheless, an important point to keep in mind is that a normalization constraint must be fulfilled and, more importantly, that we want to build a minimizing sequence of the energy functional. Therefore, as precise before, the imaginary time parametrizes the optimization algorithm at the continuous level. As a consequence, a suitable scheme must produce a minimizing sequence, at least in some situations (e.g. for $f:=0$ ). In [22], Bao \& Du analyze a few a priori standard schemes for 25]. The conclusion is the following. The time splitting scheme (see Section 4.2, page 54, for the real time-domain GPE) which is generally an efficient and accurate method in computational dynamics must not be used here since the time step required to get a decaying energy is too small. This property can be observed even in simple situations, for example for the non rotating case $(\Omega=0)$. 
Another solution consists in applying the unconditionally stable Crank-Nicolson $(\mathrm{CN})$ scheme that has the a priori advantage of being second-order accurate both in space and time. The difficulty is that this scheme is extremely time consuming since it requires the accurate solution to a nonlinear PDE at each time step. A possibility consists in writing in an explicit way the nonlinear term (semi-implicit scheme) resulting in the solution of a linear (and not nonlinear) system at each time step. Even if this solution seems attractive, the associated sequence is minimizing if a strong restrictive CFL (Courant-Friedrichs-Lewy) condition between the time and spatial steps holds. Concerning the backward Euler scheme (and similarly to the CN scheme), a nonlinear system must also be solved at each time step. However, the very nice result obtained by Bao \& Du [22] is that the semi-implicit backward Euler scheme (see section 2.2.1 produces a minimizing sequence without any CFL condition, unlike the $\mathrm{CN}$ scheme. This property holds when the potential $V$ is positive.

Concerning the spatial discretization, we consider two approaches (section 2.2.2). The first one consists in simply choosing a standard second-order finite difference scheme. An alternative discretization is the pseudo-spectral scheme based on Fast Fourier Transforms (FFTs). The reason why this last choice is seducing is that the resulting CNGF method is very robust while also being simple. Indeed, it leads to the accurate computation of the stationary states even for GPEs with large rotation speeds $\Omega$. These states cannot be reached when considering low-order spatial discretization schemes (second-order for example). Finally, the efficiency of the FFT algorithms on large clusters of HPC can lead to the possibility of computing extremely complex $3 \mathrm{~d}$ BECs configurations, based on CPU, GPU or hybrid computers.

\subsubsection{Semi-implicit backward Euler scheme in time}

Let us introduce the semi-implicit Euler scheme [22] (which is a reference scheme in the sequel). We consider a uniform time discretization: $\delta t_{n}=\delta t=t_{n+1}-t_{n}, \forall n \in \mathbb{N}$, and obtain the semi-discrete time scheme for CNGF

$$
\left\{\begin{array}{l}
\frac{\tilde{\phi}(\mathbf{x})-\phi\left(\mathbf{x}, t_{n}\right)}{\delta t}=\frac{1}{2} \Delta \tilde{\phi}(\mathbf{x})+\Omega L_{z} \tilde{\phi}(\mathbf{x})-V(\mathbf{x}) \tilde{\phi}(\mathbf{x}) \\
\quad-f\left(\left|\phi\left(\mathbf{x}, t_{n}\right)\right|^{2}\right) \tilde{\phi}(\mathbf{x}), \quad \forall \mathbf{x} \in \mathbb{R}^{d}, \\
\phi\left(\mathbf{x}, t_{n+1}\right)=\frac{\tilde{\phi}(\mathbf{x})}{\|\tilde{\phi}\|_{L_{\mathbf{x}}^{2}}}, \\
\phi(\mathbf{x}, 0)=\phi_{0}(\mathbf{x}), \text { with }\left\|\phi_{0}\right\|_{L_{\mathbf{x}}^{2}}=1 .
\end{array}\right.
$$

The reason why this scheme is considered as a "good" discretization scheme for CNGF is a consequence of the following Theorem (Bao \& Du [22]).

Theorem 1 Let us assume that $V(\mathbf{x}) \geq 0, \forall \mathbf{x} \in \mathbb{R}^{d}, \Omega=0$ and $f\left(|\phi|^{2}\right)=\beta|\phi|^{2}$. Then, for any $\beta \geq 0$, the following results hold: $\forall n \in \mathbb{N}$, 


$$
\left\|\phi\left(\mathbf{x}, t_{n}\right)\right\|_{L_{\mathbf{x}}^{2}}=\left\|\phi_{0}\right\|_{L_{\mathbf{x}}^{2}}=1
$$

and

$$
\mathscr{E}_{\Omega, f}(\tilde{\phi}(\cdot)) \leq \mathscr{E}_{\Omega, f}\left(\phi\left(\cdot, t_{n}\right)\right) .
$$

Theorem 1 confirms that the semi-implicit Euler discretization scheme leads to a decaying modified energy $\mathscr{E}_{\Omega, f}$ at each step of the projected steepest descent algorithm.

To numerically check that the numerical solution converged to the stationary state, we consider in the sequel the following (strong) criterion

$$
\left\|\phi\left(\cdot, t_{n+1}\right)-\phi\left(\cdot, t_{n}\right)\right\|_{\infty}<\varepsilon \delta t,
$$

where $\|\cdot\|_{\infty}$ is the infinity norm. We need to fix $\varepsilon$ small enough to obtain a good accuracy of the stationary state, most particularly when considering highly accurate solutions based on pseudo-spectral approximation techniques. Let us remark that we may also choose another (weak) stopping criterion that is associated with the evolution of the energy

$$
\left|\mathscr{E}_{\Omega, F}\left(\phi\left(\cdot, t_{n+1}\right)\right)-\mathscr{E}_{\Omega, F}\left(\phi\left(\cdot, t_{n}\right)\right)\right|<\varepsilon \delta t .
$$

This second criterion is defined in GPELab.

\subsubsection{Spatial discretizations}

We now focus on the spatial discretization of system (27). We consider the case of the dimension $d=2$, the generalization to $d=1$ and $d=3$ being direct by adapting the notations. Since problem 27] is set in the whole space, the computational domain has to be truncated. Because there is no physical boundary, it is natural to choose a rectangular computational domain $\mathscr{O}:=]-a_{x}, a_{x}[\times]-a_{y}, a_{y}[$. We consider a uniform discretization grid for $\mathscr{O}$ : for any indices $J(\geq 3)$ and $K(\geq 3)$ in $\mathbb{N}$, we define

$$
\mathscr{O}_{J, K}=\left\{\mathbf{x}_{j, k}=\left(x_{j}, y_{k}\right) \in \mathscr{O}, \quad \forall j \in\{0, \ldots, J\} \text { and } \forall k \in\{0, \ldots, K\}\right\},
$$

with $h_{x}=x_{j+1}-x_{j}, \forall j \in\{0, \ldots, J-1\}$, and $h_{y}=y_{k+1}-y_{k}, \forall k \in\{0, \ldots, K-$ 1\}. We introduce: $x_{0}=-a_{x}, x_{J}=a_{x}, y_{0}=-a_{y}$ and $y_{K}=a_{y}$. Furthermore, we define the set of indices: $\mathscr{F}_{J, K}=\left\{(j, k) \in \mathbb{N}^{2} ; 1 \leq j \leq J-1\right.$ and $\left.1 \leq k \leq K-1\right\}$, for finite difference schemes with a Dirichlet boundary condition, and $\mathscr{P}_{J, K}=$ $\left\{(j, k) \in \mathbb{N}^{2} ; 1 \leq j \leq J\right.$ and $\left.1 \leq k \leq K\right\}$, for the pseudo-spectral approximation with periodic boundary condition.

Finite difference discretization. We give the discretization of the operators appearing in problem (27) when using finite differences. We assume that the potential $V$ confines the stationary states in $\mathscr{O}$ (which is physically realistic) and that we can choose a Dirichlet boundary condition, i.e. $\tilde{\phi}(\mathbf{x})=0$, for $\mathbf{x} \in \partial \mathscr{O}$. For any function $\varphi$ defined on the grid $\mathscr{O}_{J, K}$, we set: $\varphi\left(\mathbf{x}_{j, k}\right)=\varphi\left(x_{j}, y_{k}\right)=\varphi_{j, k}$, for points $\mathbf{x}_{j, k}$ in the 
computational grid, $j \in\{1, \ldots, J-1\}, k \in\{1, \ldots, K-1\}$, considering the Dirichlet boundary condition. Concerning the directional derivatives along $x$ or $y$, we use the second-order approximations

$$
\forall(j, k) \in \mathscr{F}_{J, K}, \quad \delta_{x} \phi_{j, k}=\frac{\phi_{j+1, k}-\phi_{j-1, k}}{2 h_{x}}, \quad \delta_{y} \phi_{j, k}=\frac{\phi_{j, k+1}-\phi_{j, k-1}}{2 h_{y}} .
$$

Since we impose a Dirichlet boundary condition, we have: $\forall(j, k) \in \mathscr{F}_{J, K}$

$$
\delta_{x} \phi_{1, k}=\frac{\phi_{2, k}}{2 h_{x}}, \quad \delta_{y} \phi_{j, 1}=\frac{\phi_{j, 2}}{2 h_{y}}, \quad \delta_{x} \phi_{J-1, k}=-\frac{\phi_{J-2, k}}{2 h_{x}}, \quad \delta_{y} \phi_{j, K-1}=-\frac{\phi_{j, K-2}}{2 h_{y}} .
$$

Consequently, the second-order discretization of the rotation operator $L_{z}$ is

$$
\forall(j, k) \in \mathscr{F}_{J, K},\left(\left[L_{z}\right] \phi\right)_{j, k}:=-i\left(x_{j} \delta_{y} \phi_{j, k}-y_{k} \delta_{x} \phi_{j, k}\right) .
$$

If $L=(J-1)(K-1)$, we associate the matrix $\left[L_{z}\right] \in \mathscr{M}_{L}(\mathbb{C})$ to this discrete operator and we denote by $\phi:=\left(\phi_{I(j, k)}\right)_{(j, k) \in \mathscr{F}_{J, K}}$ the unknown vector in $\mathbb{C}^{L}$, where we assume that the indices ordering is such that: $I(j, k)=j+(J-1)(k-1)$, and $\phi_{I(j, k)}=\phi_{j, k}$.

Concerning the derivatives of order two, we use the second-order centered threepoints formulae in the directions $x$ and $y: \forall(j, k) \in \mathscr{F}_{J, K}$

$$
\delta_{x}^{2} \phi_{j, k}=\frac{\phi_{j+1, k}-2 \phi_{j, k}+\phi_{j-1, k}}{h_{x}^{2}}, \quad \delta_{y}^{2} \phi_{j, k}=\frac{\phi_{j, k+1}-2 \phi_{j, k}+\phi_{j, k-1}}{h_{y}^{2}} .
$$

Since we consider a Dirichlet boundary condition, we have: $\forall(j, k) \in \mathscr{F}_{J, K}$

$$
\begin{aligned}
& \delta_{x}^{2} \phi_{1, k}=\frac{\phi_{2, k}-2 \phi_{1, k}}{h_{x}^{2}}, \quad \delta_{y}^{2} \phi_{j, 1}=\frac{\phi_{j, 2}-2 \phi_{j, 1}}{h_{y}^{2}}, \\
& \delta_{x}^{2} \phi_{J-1, k}=\frac{-2 \phi_{J-1, k}+\phi_{J-2, k}}{h_{x}^{2}}, \quad \delta_{y} \phi_{j, K-1}=\frac{-2 \phi_{j, K-1}+\phi_{j, K-2}}{h_{y}^{2}} .
\end{aligned}
$$

The Laplacian operator $\Delta$ is then classically discretized by the five-points finite difference scheme

$$
\forall(j, k) \in \mathscr{F}_{J, K}, \quad([\Delta] \phi)_{j, k}=\delta_{x}^{2} \phi_{j, k}+\delta_{y}^{2} \phi_{j, k} .
$$

We associate the matrix $[\Delta] \in \mathscr{M}_{L}(\mathbb{C})$ to this discrete operator.

The potential and nonlinear operators are pointwise evaluated: $\forall(j, k) \in \mathscr{F}_{J, K}$

$$
([V] \phi)_{j, k}=V\left(\mathbf{x}_{j, k}\right) \phi_{j, k} \text { and }\left(\left[f\left(\left|\phi^{n}\right|^{2}\right)\right] \phi\right)_{j, k}=f\left(\left|\phi_{j, k}^{n}\right|^{2}\right) \phi_{j, k} .
$$

The matrices $[V] \in \mathscr{M}_{L}(\mathbb{C})$ and $\left[f\left(\left|\phi^{n}\right|^{2}\right)\right] \in \mathscr{M}_{L}(\mathbb{C})$ are diagonal after the indices reordering.

Finally, the finite difference discretization of problem 27) leads to the finite dimensional approximation: compute the sequence of vector fields $\left(\phi^{n}\right)_{n \in \mathbb{N}}$ in $\mathbb{C}^{L}$ 
through

$$
\left\{\begin{array}{l}
\mathbb{A}_{\mathrm{FD}}^{\mathrm{BE}, n} \tilde{\phi}=\mathbf{b}^{n}, \\
\phi^{n+1}=\frac{\tilde{\phi}}{\|\tilde{\phi}\|_{\ell_{0}^{2}}}, \\
\phi^{0}=\phi_{0},
\end{array}\right.
$$

with

$$
\left\{\begin{array}{l}
\mathbb{A}_{\mathrm{FD}}^{\mathrm{BE}, n}:=\frac{1}{\delta t}[I]-\frac{1}{2}[\Delta]-\Omega\left[L_{z}\right]+[V]+\left[f\left(\left|\phi^{n}\right|^{2}\right)\right], \\
\mathbf{b}^{n}:=\frac{\phi^{n}}{\delta t} .
\end{array}\right.
$$

In the above system, $[I]$ is the identity matrix in $\mathscr{M}_{L}(\mathbb{C})$. The initial data $\phi^{0}$ is fixed by the values of $\phi_{0}$ at the grid points. In the framework of Dirichlet boundary conditions, we define the 2 -norm $\|\cdot\|_{\ell_{0}^{2}}$ of a complex-valued vector $\phi \in \mathbb{C}^{L}$ by

$$
\|\phi\|_{\ell_{0}^{2}}:=h_{x}^{1 / 2} h_{y}^{1 / 2}\left(\sum_{(j, k) \in \mathscr{F}_{J, K}}\left|\phi_{I(j, k)}\right|^{2}\right)^{1 / 2} .
$$

Furthermore, we define the discrete (strong) stopping criterion as

$$
\left\|\phi^{n+1}-\phi^{n}\right\|_{\infty}<\varepsilon \delta t,
$$

with the discrete uniform norm defined by: $\forall \phi \in \mathbb{C}^{L},\|\phi\|_{\infty}=\max _{(j, k) \in \mathscr{F}_{J K}}\left|\phi_{I(j, k)}\right|$, and the discrete (weak) stopping criterion as

$$
\left|\mathscr{E}_{\Omega, F}\left(\phi^{n+1}\right)-\mathscr{E}_{\Omega, F}\left(\phi^{n}\right)\right|<\varepsilon \delta t,
$$

with the discrete energy

$$
\begin{aligned}
& \mathscr{E}_{\Omega, F}(\phi)= \\
& \quad\left(h_{x} h_{y}\right)^{1 / 2} \sum_{(j, k) \in \mathscr{F}_{J, K}} \operatorname{Re}\left\{\phi_{I(j, k)}^{*}\left(-\frac{1}{2}[\Delta] \phi-\Omega\left[L_{z}\right] \phi+[V] \phi+\left[F\left(|\phi|^{2}\right)\right] \phi\right)_{j, k}\right\} .
\end{aligned}
$$

In the sequel, the discretization scheme (36) is called BEFD (for Backward Euler Finite Difference). This scheme produces a minimizing sequence $\left(\phi^{n}\right)_{n \in \mathbb{N}}$ of the modified energy under the assumptions of Theorem 11, without CFL condition, and with second-order accuracy in space.

Pseudo-spectral discretization. Let us now consider the pseudo-spectral approximation scheme based on FFTs. We still assume that the state is localized in the box $\mathscr{O}$. Unlike finite differences, we consider a periodic boundary condition which is satisfied since the function a priori vanishes on the boundary. If one chooses a Dirichlet boundary condition, then Fast Sine Transforms must be used. For a Neumann boundary condition, Fast Cosine Transforms must be applied. Nevertheless, these two last transforms require to be correctly coded through FFTs to be efficient. For example, these two methods are not included in the basic version of Matlab (but are defined in the signal processing toolbox) contrary to the FFT (which is a 
compiled version of FFT3W). Since GPELab is developed under the basic Matlab version, we restrict our study to the FFT-based algorithm.

In this framework, a function $\varphi$ (that can be considered as an approximation of the solution $\tilde{\phi}$ of problem (27) ) is defined on the uniform grid $\mathscr{O}_{J, K}$ by $\varphi_{j, k}$, for any indices $(j, k) \in \mathscr{P}_{J, K}$, i.e. excluding $j=0$ and $k=0$. Let $M:=J K$ be the number of degrees of freedom for the periodic boundary-value problem. Let us introduce $\varphi:=\left(\varphi_{j, k}\right)_{(j, k) \in \mathscr{P}_{J, K}}$, that is, $\varphi \in \mathbb{C}^{M}$ by a lexicographic reordering (that we do not precise for conciseness). The approximate pseudo-spectral approximations of a function $\phi$ in the $x$ - and $y$-directions (which is represented on $\mathscr{O}_{J, K}$ ) are respectively based on truncated partial inverse Fourier series representations: $\forall(j, k) \in \mathscr{P}_{J, K}$,

$$
\begin{aligned}
& \phi\left(t, x_{j}, y_{k}\right) \approx \varphi\left(x_{j}, y_{k}, t\right)=\frac{1}{J} \sum_{p=-J / 2}^{J / 2-1} \widehat{\varphi_{p}}\left(y_{k}, t\right) e^{i \mu_{p}\left(x_{j}+a_{x}\right)}, \\
& \phi\left(t, x_{j}, y_{k}\right) \approx \varphi\left(x_{j}, y_{k}, t\right)=\frac{1}{K} \sum_{q=-K / 2}^{K / 2-1} \widehat{\varphi_{q}}\left(x_{k}, t\right) e^{i \lambda_{q}\left(y_{k}+a_{y}\right)},
\end{aligned}
$$

where $\widehat{\varphi_{p}}$ and $\widehat{\varphi_{q}}$ are respectively the Fourier coefficients of the function $\varphi$ in the directions $x$ and $y$, the Fourier multipliers being: $\mu_{p}=\frac{\pi p}{a_{x}}$ and $\lambda_{q}=\frac{\pi q}{a_{y}}$. The functions $\widehat{\varphi_{p}}$ and $\widehat{\varphi_{q}}$ can be expressed as

$$
\begin{aligned}
\widehat{\varphi_{p}}\left(y_{k}, t\right) & =\sum_{j=0}^{J-1} \varphi\left(x_{j}, y_{k}, t\right) e^{-i \mu_{p}\left(x_{j}+a_{x}\right)}, \\
\widehat{\varphi_{q}}\left(x_{j}, t\right) & =\sum_{k=0}^{K-1} \varphi\left(x_{j}, y_{k}, t\right) e^{-i \lambda_{q}\left(y_{k}+a_{y}\right)} .
\end{aligned}
$$

Consequently, the effect of a directional derivative along $x$ or $y$, respectively, is written under the form, $\forall(j, k) \in \mathscr{P}_{J, K}$,

$$
\begin{aligned}
& \left(\left[\left[\partial_{x}\right]\right] \varphi\right)_{j, k}=\frac{1}{J} \sum_{p=-J / 2}^{J / 2-1} i \mu_{p} \widehat{\varphi_{p}}\left(y_{k}, t\right) e^{i \mu_{p}\left(x_{j}+a_{x}\right)}, \\
& \left(\left[\left[\partial_{y}\right]\right] \varphi\right)_{j, k}=\frac{1}{K} \sum_{q=-K / 2}^{K / 2-1} i \lambda_{q} \widehat{\varphi_{q}}\left(x_{k}, t\right) e^{i \lambda_{q}\left(y_{k}+a_{y}\right)} .
\end{aligned}
$$

Hence, we deduce the following approximation of the rotational operator $L_{z}$ on $\mathscr{O}_{J, K}$

$$
\left(\left[\left[L_{z}\right]\right] \varphi\right)_{j, k}=-i\left(x_{j}\left(\left[\left[\partial_{y}\right]\right] \varphi\right)_{j, k}-y_{k}\left(\left[\left[\partial_{x}\right]\right] \varphi\right)_{j, k}\right) .
$$

The formal applications of derivatives to the previous representations yield the approximations of the second-order derivatives: $\forall(j, k) \in \mathscr{P}_{J, K}$, 


$$
\begin{aligned}
& \left(\left[\left[\partial_{x}^{2}\right]\right] \varphi\right)_{j, k}=\frac{1}{J} \sum_{p=-J / 2}^{J / 2-1}-\mu_{p}^{2} \widehat{\varphi_{p}}\left(y_{k}, t\right) e^{i \mu_{p}\left(x_{j}+a_{x}\right)} \\
& \left(\left[\left[\partial_{y}^{2}\right]\right] \varphi\right)_{j, k}=\frac{1}{K} \sum_{q=-K / 2}^{K / 2-1}-\lambda_{q}^{2} \widehat{\varphi_{q}}\left(x_{k}, t\right) e^{i \lambda_{q}\left(y_{k}+a_{y}\right)},
\end{aligned}
$$

leading to the approximation of the Laplacian operator $\Delta$

$$
([[\Delta]] \varphi)_{j, k}=\left(\left[\left[\partial_{x}^{2}\right]\right] \varphi+\left[\left[\partial_{y}^{2}\right]\right] \varphi\right)_{j, k} .
$$

The potential and nonlinear operators are given pointwise, $\forall(j, k) \in \mathscr{P}_{J, K}$,

$$
([[V]] \varphi)_{j, k}=V\left(\mathbf{x}_{j, k}\right) \varphi_{j, k} \quad \text { and } \quad\left(\left[\left[f\left(\left|\phi^{n}\right|^{2}\right)\right]\right] \varphi\right)_{j, k}=f\left(\left|\phi_{j, k}^{n}\right|^{2}\right) \varphi_{j, k} .
$$

The pseudo-spectral approximation of 27] then produces a sequence of vectors $\left(\phi^{n}\right)_{n \in \mathbb{N}}$ solution to

$$
\left\{\begin{array}{l}
\mathbb{A}_{\mathrm{SP}}^{\mathrm{BE}, n} \tilde{\phi}=\mathbf{b}^{\mathrm{BE}, n}, \\
\phi^{n+1}=\frac{\tilde{\phi}}{\|\tilde{\phi}\|_{\ell_{\pi}^{2}}}, \\
\phi^{0}:=\phi_{0},
\end{array}\right.
$$

where $\tilde{\phi} \in \mathbb{C}^{M}$. The right-hand side is

$$
\mathbf{b}^{\mathrm{BE}, n}:=\frac{\phi^{n}}{\delta t},
$$

with $\phi^{n} \in \mathbb{C}^{M}$. The map $\|\cdot\|_{\ell_{\pi}^{2}}$ corresponds to the discrete $L_{\mathbf{x}}^{2}$-norm on the grid $\mathscr{O}_{J, K}$ for a vector $\phi \in \mathbb{C}^{M}$

$$
\|\phi\|_{\ell_{\pi}^{2}}:=h_{x}^{1 / 2} h_{y}^{1 / 2}\left(\sum_{(j, k) \in \mathscr{P}_{J, K}}\left|\phi_{j, k}\right|^{2}\right)^{1 / 2}
$$

Furthermore, we define the discrete (strong) stopping criterion as

$$
\left\|\phi^{n+1}-\phi^{n}\right\|_{\infty}<\varepsilon \delta t,
$$

with the discrete uniform norm defined by: $\forall \phi \in \mathbb{C}^{M},\|\phi\|_{\infty}=\max _{(j, k) \in \mathscr{P}_{J, K}}\left|\phi_{j, k}\right|$, and the discrete (weak) stopping criterion as

$$
\left|\mathscr{E}_{\Omega, F}\left(\phi^{n+1}\right)-\mathscr{E}_{\Omega, F}\left(\phi^{n}\right)\right|<\varepsilon \delta t,
$$

with the discrete energy

$$
\begin{aligned}
& \mathscr{E}_{\Omega, F}(\phi)= \\
& \quad\left(h_{x} h_{y}\right)^{1 / 2} \sum_{(j, k) \in \mathscr{P}_{J, K}} \operatorname{Re}\left\{\phi_{j, k}^{*}\left(-\frac{1}{2}[[\Delta]] \phi-\Omega\left[\left[L_{z}\right]\right] \phi+[[V]] \phi+\left[\left[F\left(|\phi|^{2}\right)\right]\right] \phi\right)_{j, k}\right\} .
\end{aligned}
$$


In $\sqrt{45}$, the operator $\mathbb{A}_{\mathrm{SP}}^{\mathrm{BE}, n}$ is a map which, for any vector $\phi \in \mathbb{C}^{M}$, associates a vector $\psi \in \mathbb{C}^{M}$ such that

$$
\begin{aligned}
& \psi:=\mathbb{A}_{\mathrm{SP}}^{\mathrm{BE}, n} \phi=\mathbb{A}_{\mathrm{TF}}^{\mathrm{BE}, n} \psi+\mathbb{A}_{\Delta, \Omega}^{\mathrm{BE}} \phi, \\
& \mathbb{A}_{\mathrm{TF}}^{\mathrm{BE}, n} \phi:=\left(\frac{[[I]]}{\delta t}+[[V]]+\left[\left[f\left(\left|\phi^{n}\right|^{2}\right)\right]\right]\right) \phi, \\
& \mathbb{A}_{\Delta, \Omega}^{\mathrm{BE}} \phi:=\left(-\frac{1}{2}[[\Delta]]-\Omega\left[\left[L_{z}\right]\right]\right) \phi,
\end{aligned}
$$

where $[[I]]$ is the identity matrix of $\mathscr{M}_{M}(\mathbb{C})$.

To evaluate the operator $\mathbb{A}_{\mathrm{TF}}^{\mathrm{BE}, n}$, we use 44 . We remark that the operator is diagonal in the physical space. For $\mathbb{A}_{\Delta, \Omega}^{\mathrm{BE}}$, we consider 442 and 443 for $\left[\left[L_{z}\right]\right]$ and $[[\Delta]]$, respectively. Let us note that $\mathbb{A}_{\Delta, \Omega}^{\mathrm{BE}}$ is not diagonal in the physical space but $[[\Delta]]$, defined by [43), is diagonal in the Fourier space. The semi-implicit backward Euler scheme with a pseudo-spectral approximation is now designated by BESP (for Backward Euler pseudo-SPectral).

\subsubsection{Fully discretized semi-implicit Crank-Nicolson scheme}

The discretization of (25) by using the semi-implicit Crank-Nicolson scheme is

$$
\left\{\begin{array}{l}
\frac{\tilde{\phi}(\mathbf{x})-\phi\left(\mathbf{x}, t_{n}\right)}{\delta t}=\frac{1}{2} \Delta\left(\frac{\tilde{\phi}(\mathbf{x})+\phi\left(\mathbf{x}, t_{n}\right)}{2}\right)+\Omega L_{z}\left(\frac{\tilde{\phi}(\mathbf{x})+\phi\left(\mathbf{x}, t_{n}\right)}{2}\right) \\
\quad-V(\mathbf{x})\left(\frac{\tilde{\phi}(\mathbf{x})+\phi\left(\mathbf{x}, t_{n}\right)}{2}\right)-f\left(\left|\phi\left(\mathbf{x}, t_{n}\right)\right|^{2}\right)\left(\frac{\tilde{\phi}(\mathbf{x})+\phi\left(\mathbf{x}, t_{n}\right)}{2}\right), \forall \mathbf{x} \in \mathbb{R}^{d}, \\
\phi\left(\mathbf{x}, t_{n+1}\right)=\frac{\tilde{\phi}(\mathbf{x})}{\|\tilde{\phi}(\mathbf{x})\|_{L_{\mathbf{x}}^{2}}}, \\
\phi^{0}=\phi_{0}, \text { with }\left\|\phi_{0}\right\|_{L_{\mathbf{x}}^{2}}=1 .
\end{array}\right.
$$

In [22], Bao \& Du proved that the scheme (50) for the one-dimensional case generates a minimizing sequence of the energy functional under some assumptions similar to Theorem 1 , with $\beta=0$, but with the following strong CFL constraint

$$
\delta t \leq \frac{2 h_{x}^{2}}{2+h_{x}^{2} \max _{j \in\{1, \ldots, J-1\}} V\left(x_{j}\right)},
$$

for a uniform finite difference discretization $\left(x_{j}\right)_{j \in\{1, \ldots, J-1\}}$, with spatial step $h_{x}$, on an interval $]-a_{x}, a_{x}[$. In a practical computation, this CFL is very restrictive.

Concerning the spatial discretization, the previous approaches (FD and SP) directly extend. For example, for the finite difference scheme at the iteration $n$, we obtain the CNFD scheme

$$
\left\{\begin{array}{l}
\mathbb{A}_{\mathrm{FD}}^{\mathrm{CN}, n} \tilde{\phi}=\mathbf{b}^{n}, \\
\phi^{n+1}=\frac{\tilde{\phi}}{\|\tilde{\phi}\|_{\ell_{0}^{2}}},
\end{array}\right.
$$


with

$$
\begin{aligned}
& \mathbb{A}_{\mathrm{FD}}^{\mathrm{CN}, n}:=\frac{1}{\delta t}[I]+\frac{1}{2}\left(-\frac{1}{2}[\Delta]-\Omega\left[L_{z}\right]+[V]+\left[f\left(\left|\phi^{n}\right|^{2}\right)\right]\right), \\
& \mathbf{b}^{n}:=\frac{\phi^{n}}{\delta t}+\frac{1}{2}\left(\frac{1}{2}[\Delta]+\Omega\left[L_{z}\right]-[\mathbb{V}]-\left[f\left(\left|\phi^{n}\right|^{2}\right)\right]\right) \phi^{n},
\end{aligned}
$$

and the initial data $\phi^{0}=\phi_{0} \in \mathbb{C}^{L}$.

For the pseudo-spectral approximation at iteration $n$, the CNSP scheme is

$$
\left\{\begin{array}{l}
\mathbb{A}_{\mathrm{SP}}^{\mathrm{CN}, n} \tilde{\phi}=\mathbf{b}^{\mathrm{CN}, n} \\
\phi^{n+1}=\frac{\tilde{\phi}}{\|\tilde{\phi}\|_{\ell_{\pi}^{2}}}
\end{array}\right.
$$

where $\mathbb{A}_{\mathrm{SP}}^{\mathrm{CN}, n}$ is the operator which maps any vector $\phi \in \mathbb{C}^{M}$ to $\psi \in \mathbb{C}^{M}$ through the relations

$$
\begin{aligned}
& \psi:=\mathbb{A}_{\mathrm{SP}}^{\mathrm{CN}, n} \phi=\mathbb{A}_{\mathrm{TF}}^{\mathrm{CN}, n} \phi+\mathbb{A}_{\Delta, \Omega}^{\mathrm{CN}, n} \phi, \\
& \mathbb{A}_{\mathrm{TF}}^{\mathrm{CN}, n} \phi:=\left(\frac{[[I]]}{\delta t}+\frac{1}{2}[[V]]+\frac{1}{2}\left[\left[f\left(\left|\phi^{n}\right|^{2}\right)\right]\right]\right) \phi, \\
& \mathbb{A}_{\Delta, \Omega}^{\mathrm{CN}} \phi:=\left(-\frac{1}{4}[[\Delta]]-\frac{1}{2} \Omega\left[\left[L_{z}\right]\right]\right) \phi .
\end{aligned}
$$

The right-hand side is

$$
\mathbf{b}^{\mathrm{CN}, n}:=\left(\frac{[[I]]}{\delta t}+\frac{1}{2}\left(\frac{1}{2}[[\Delta]]+\Omega\left[\left[L_{z}\right]\right]-[[V]]-\left[\left[f\left(\left|\phi^{n}\right|^{2}\right)\right]\right]\right)\right) \phi^{n} .
$$

Like for the semi-implicit Euler scheme, we remark that $\mathbb{A}_{\mathrm{TF}}^{\mathrm{CN}, n}$ is diagonal in the physical space and $\mathbb{A}_{\Delta, 0}$ is also diagonal but in the Fourier space.

\subsubsection{BESP or CNSP? That is the question}

In Sections 2.2.1, 2.2.2 and 2.2.3, we introduced the BESP and CNSP schemes that correspond to the semi-implicit Euler and Crank-Nicolson schemes for a pseudospectral spatial discretization. We have seen that BESP diminishes the energy without any CFL condition between the time and spatial steps while the CNSP scheme is constrained. We illustrate here through a numerical example that the constraint related to CNSP makes it useless for computing a stationary state while BESP is robust. A similar conclusion applies to BEFD and CNFD.

Let us consider the two-dimensional problem

$$
\left\{\begin{array}{l}
i \partial_{t} \psi(t, \mathbf{x})=-\frac{1}{2} \Delta \psi(t, \mathbf{x})-\Omega L_{z} \psi(t, \mathbf{x})+V(\mathbf{x}) \psi(t, \mathbf{x})+\beta|\psi|^{2} \psi(t, \mathbf{x}), \\
\psi(0, \mathbf{x})=\psi_{0}(\mathbf{x}) \in L_{\mathbf{x}}^{2}
\end{array}\right.
$$

for $t>0$ and $\mathbf{x} \in \mathbb{R}^{2}$. The potential is harmonic: $V(\mathbf{x})=\frac{1}{2}\left(\gamma_{x}^{2} x^{2}+\gamma_{y}^{2} y^{2}\right)$, with $\gamma_{x}=$ $\gamma_{y}=1$. Moreover, we assume that: $\beta \in \mathbb{R}^{+}$and $\Omega \in \mathbb{R}$. We consider BESP and CNSP 
for $\delta t=10^{-1}$ to show the behavior of the associated energy. When using BESP and CNSP, each iteration $n$ requires the solution to a linear system by a Krylov subspace iterative solver (see Section 2.4). The computational domain is: $\mathscr{O}=]-10,10\left[{ }^{2}\right.$, for a uniform grid $\mathscr{O}_{J, K}$, with $J=K=2^{9}$. The initial data is chosen as the ThomasFermi approximation 66 when $\beta \neq 0$ and the centered gaussian

$$
\phi_{\mathrm{osc}}(\mathbf{x})=\frac{\left(\gamma_{x} \gamma_{y}\right)^{1 / 4}}{\sqrt{\pi}} e^{-\left(\gamma_{x} x^{2}+\gamma_{y} y^{2}\right) / 2},
$$

for $\beta=0$.

We report on Figures 3(a) 3(b) the evolution of the energy $\Delta \mathscr{E}_{\Omega, F}=\mathscr{E}_{\Omega, F}\left(\phi^{1}\right)-$ $\mathscr{E}_{\Omega, F}\left(\phi^{0}\right)$ for BESP and CNSP for the first time step with respect to $\beta$ and $\Omega$. We observe that the energy decays for BESP in all cases. However, the energy increases for CNSP, leading to the divergence of the scheme almost immediately (this is worst for large values of $\beta$ ). To illustrate the difference between these two schemes, we draw on Figures 4(a) and 4(b) the evolution of the energy for BESP and CNSP, respectively, until $T=1$ for the time steps $\delta t=10^{-1}, 10^{-2}$ and $10^{-3}$, with $\beta=500$ and $\Omega=0.5$. For BESP, the energy decays for the three time steps. We can also see that the energy decays faster as the time step is smaller. Concerning CNSP, we observe that the method diverges for $\delta t=10^{-1}$ since the energy increases. For the time steps $\delta t=10^{-2}$ and $10^{-3}$, the energy decays smoothly all along the simulation similarly to BESP. To have a diminishing energy, a significantly smaller time step must be chosen, limiting hence the application range of CNSP most particularly in terms of convergence rate towards the minimum. For this reason, BESP is a robust scheme. Other simulations support this conclusion for $\Omega>0$. Finally, only BESP and BEFD are considered in the sequel.

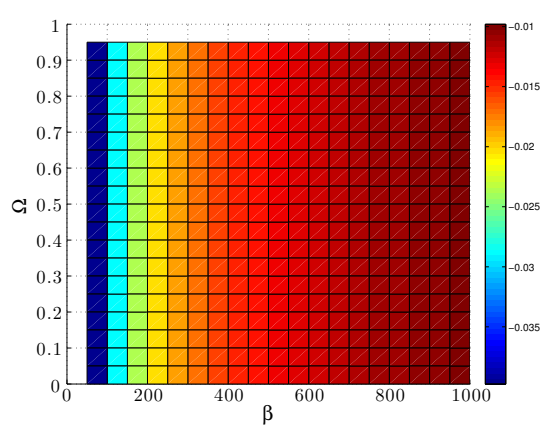

(a) Evolution of the energy for BESP

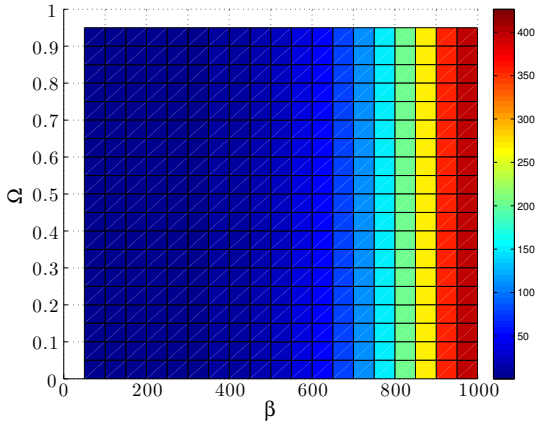

(b) Evolution of the energy for CNSP

Fig. 3 Evolution of the energy for the first time step for BESP and CNSP. 


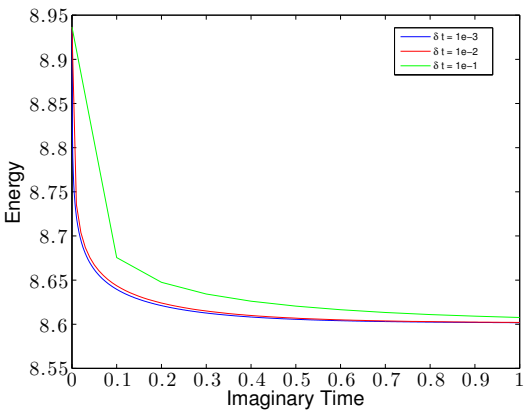

(a) Evolution of the energy for BESP and different $\delta t$

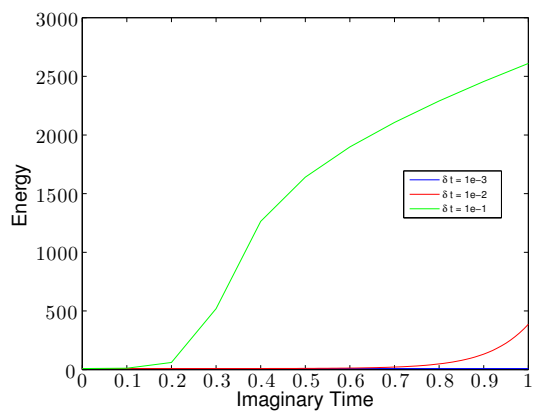

(b) Evolution of the energy for CNSP and different $\delta t$

Fig. 4 Evolution of the energy until $T=1$ for BESP and CNSP with $\beta=500$ and $\Omega=0.5$.

\subsubsection{BESP or BEFD? This is another question}

We analyze now the spatial accuracy of BESP and BEFD. In particular, we show that there is a great interest in considering the pseudo-spectral approximation rather than the finite difference scheme. A similar study has been conducted by Bao, Chern \& $\operatorname{Lim}$ [21], for $\Omega=0$, where the authors show that BESP provides a spectral precision compared with BEFD.

We first consider a numerical test similar to [21]. The problem is

$$
\left\{\begin{array}{l}
i \partial_{t} \psi(t, x)=-\frac{1}{2} \partial_{x}^{2} \psi(t, \mathbf{x})+V(x) \psi(t, x)+\beta|\psi|^{2} \psi(t, x), \\
\psi(0, x)=\psi_{0}(x) \in L_{x}^{2}
\end{array}\right.
$$

where $V(x)=\frac{1}{2} x^{2}, \beta=300$ and $\psi_{0}$ is the centered normalized gaussian, i.e.

$$
\forall x \in \mathbb{R}, \quad \psi_{0}(x)=\frac{1}{\pi^{1 / 4}} e^{-\frac{x^{2}}{2}} .
$$

We choose the computational domain $\mathscr{O}=]-10,10[$ and the associated uniform grid $\mathscr{O}_{J}$, with $6 \leq J \leq 12$. We use BESP and BEFD for computing a stationary state of 58) on various grids. The time step is $\delta t=10^{-1}$ and the linear systems are solved by BiCGStab with a stopping criterion $\varepsilon^{\text {Krylov }}=10^{-12}$. Let $\phi_{J}^{\mathrm{SP}}$ (respectively, $\phi_{J}^{\mathrm{FD}}$ ) be the stationary state computed on $\mathscr{O}_{J}, 6 \leq J \leq 12$, with BESP (respectively, BEFD), and $\phi_{\text {ref }}^{\mathrm{SP}}=\phi_{2^{12}}^{\mathrm{SP}}\left(\right.$ respectively, $\left.\phi_{\mathrm{ref}}^{\mathrm{FD}}=\phi_{2^{12}}^{\mathrm{FD}}\right)$ the reference stationary state. We report in Table 1 the quadratic error, the infinity norm error and finally the energy norm error between the reference and computed stationary states for BESP and BEFD. We observe the spectral accuracy of the stationary states obtained with BESP and the quadratic precision of BEFD with respect to the different grids.

Let us now consider the two-dimensional example given by system (56) for the harmonic potential : $V(\mathbf{x})=\frac{1}{2}\left(\gamma_{x}^{2} x^{2}+\gamma_{y}^{2} y^{2}\right)$, with $\gamma_{x}=\gamma_{y}=1$. We fix $\beta=300$ and $\Omega=0.6$. The computational domain is $\mathscr{O}=]-10,10[2$, for a uniform spatial grid 


\begin{tabular}{|c|c|c|c|c|c|c|}
\hline & $J=2^{6}$ & $J=2^{7}$ & $J=2^{8}$ & $J=2^{9}$ & $J=2^{10}$ & $J=2^{11}$ \\
\hline$\left\|\phi_{\text {ref }}^{\mathrm{SP}}-\phi_{J}^{\mathrm{SP}}\right\|_{\infty}$ & $5.00 \mathrm{e}-5$ & $8.30 \mathrm{e}-9$ & $<1 \mathrm{e}-12$ & $<1 \mathrm{e}-12$ & $<1 \mathrm{e}-12$ & $<1 \mathrm{e}-12$ \\
\hline$\left\|\phi_{\text {ref }}^{\mathrm{FD}}-\phi_{J}^{\mathrm{FD}}\right\|_{\infty}$ & $3.21 \mathrm{e}-5$ & $2.12 \mathrm{e}-6$ & $1.33 \mathrm{e}-7$ & $8.32 \mathrm{e}-9$ & $6.03 \mathrm{e}-10$ & $1.94 \mathrm{e}-10$ \\
\hline$\left\|\phi_{\text {ref }}^{\mathrm{SP}}-\phi_{J}^{\mathrm{SP}}\right\|_{\ell_{\pi}^{2}}$ & $4.51 \mathrm{e}-5$ & $1.00 \mathrm{e}-8$ & $<1 \mathrm{e}-12$ & $<1 \mathrm{e}-12$ & $<1 \mathrm{e}-12$ & $<1 \mathrm{e}-12$ \\
\hline$\left\|\phi_{\text {ref }}^{\mathrm{FD}}-\phi_{J}^{\mathrm{FD}}\right\|_{\ell_{0}^{2}}$ & $2.99 \mathrm{e}-5$ & $1.96 \mathrm{e}-6$ & $1.23 \mathrm{e}-7$ & $7.83 \mathrm{e}-9$ & $7.15 \mathrm{e}-10$ & $1.74 \mathrm{e}-10$ \\
\hline$\left|\mathscr{E}_{\Omega, F}\left(\phi_{\text {ref }}^{\mathrm{SP}}\right)-\mathscr{E}_{\Omega, F}\left(\phi_{J}^{\mathrm{SP}}\right)\right|$ & $9.19 \mathrm{e}-5$ & $3.65 \mathrm{e}-10$ & $2.22 \mathrm{e}-12$ & $2.66 \mathrm{e}-12$ & $<1 \mathrm{e}-12$ & $1.91 \mathrm{e}-12$ \\
\hline$\left|\mathscr{E}_{\Omega, F}\left(\phi_{\text {ref }}^{\mathrm{FD}}\right)-\mathscr{E}_{\Omega, F}\left(\phi_{J}^{\mathrm{FD}}\right)\right|$ & $8.30 \mathrm{e}-6$ & $5.54 \mathrm{e}-7$ & $3.51 \mathrm{e}-8$ & $2.23 \mathrm{e}-9$ & $1.52 \mathrm{e}-10$ & $1.25 \mathrm{e}-11$ \\
\hline
\end{tabular}

Table 1 Numerical accuracy for BESP/BEFD for computing the ground state associated to 58 .

$\mathscr{O}_{J, K}$, with: $5 \leq J, K \leq 9$. The time step is $\delta t=10^{-1}$. The linear systems are solved by BiCGStab for a stopping criterion on the residual equal to $\varepsilon^{\mathrm{Krylov}}=10^{-12}$. For $J$ and $K$ varying, we can compare the different computed stationary states with a reference numerical solution $\phi_{\text {ref }}$ obtained with BESP on a fine uniform grid (here $\mathscr{O}_{J, K}$, with $\left.J=K=2^{9}\right)$. Let $\phi_{J}$ be the state calculated on a grid $\mathscr{O}_{J, K}(J=K)$. We report on Figure 5(a) 5(f) the different densities obtained for BESP and BEFD. We remark that, for coarse grids, the solutions are very different and the finite difference discretization seems to lead to the most accurate results in this case. In Table 2 , we can see that there is an improved accuracy of BESP when going from a grid with $J=2^{6}$ to a grid with $J=2^{7}$. We observe a convergence towards a different stationary state for the grids with $J \leq 2^{6}$ and the grids for $J>6$ as seen on Figures 5(a) 5(f). We see that BESP provides a high resolution calculation and the accuracy is far better than for BEFD for discretization grids with $J \geq 2^{7}$.

\begin{tabular}{|c|c|c|c|c|}
\hline & $J=2^{5}$ & $J=2^{6}$ & $J=2^{7}$ & $J=2^{8}$ \\
\hline$\left\|\phi_{\text {ref }}^{\mathrm{SP}}-\phi_{J}^{\mathrm{SP}}\right\|_{\infty}$ & $1.47 \mathrm{e}-1$ & $6.95 \mathrm{e}-2$ & $5.41 \mathrm{e}-7$ & $1.78 \mathrm{e}-9$ \\
\hline$\left\|\phi_{\text {ref }}^{\mathrm{FD}}-\phi_{J}^{\mathrm{FD}}\right\|_{\infty}$ & $1.29 \mathrm{e}-1$ & $5.02 \mathrm{e}-3$ & $6.71 \mathrm{e}-5$ & $4.39 \mathrm{e}-6$ \\
\hline$\left\|\phi_{\text {ref }}^{\mathrm{SP}}-\phi_{J}^{\mathrm{SP}}\right\|_{\ell_{\pi}^{2}}$ & $1.82 \mathrm{e}-1$ & $4.23 \mathrm{e}-2$ & $<1 \mathrm{e}-12$ & $<1 \mathrm{e}-12$ \\
\hline$\left\|\phi_{\text {ref }}^{\mathrm{FD}}-\phi_{J}^{\mathrm{FD}}\right\|_{\ell_{0}^{2}}$ & $4.77 \mathrm{e}-2$ & $6.46 \mathrm{e}-5$ & $3.79 \mathrm{e}-8$ & $1.44 \mathrm{e}-10$ \\
\hline$\left|\mathscr{E}_{\Omega, F}\left(\phi_{\text {ref }}^{\mathrm{SP}}\right)-\mathscr{E}_{\Omega, F}\left(\phi_{J}^{\mathrm{SP}}\right)\right|$ & $7.55 \mathrm{e}-3$ & $5.29 \mathrm{e}-5$ & $2.54 \mathrm{e}-8$ & $<1 \mathrm{e}-12$ \\
\hline$\left|\mathscr{E}_{\Omega, F}\left(\phi_{\text {ref }}^{\mathrm{FD}}\right)-\mathscr{E}_{\Omega, F}\left(\phi_{J}^{\mathrm{FD}}\right)\right|$ & $5.274 \mathrm{e}-2$ & $3.054 \mathrm{e}-3$ & $1.871 \mathrm{e}-4$ & $1.12 \mathrm{e}-5$ \\
\hline
\end{tabular}

Table 2 Numerical accuracy of BESP and BEFD for computing the stationary state associated with problem 56 .

To conclude, BESP is far more accurate than BEFD when fine enough grids are considered. As seen in the examples, this precision directly impacts the accuracy of the associated physical quantities. In the sequel, we focus on BESP. 


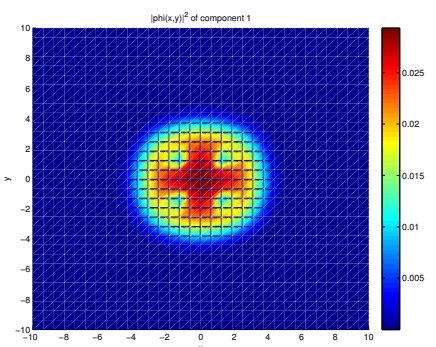

(a) BEFD: $J=2^{5}$

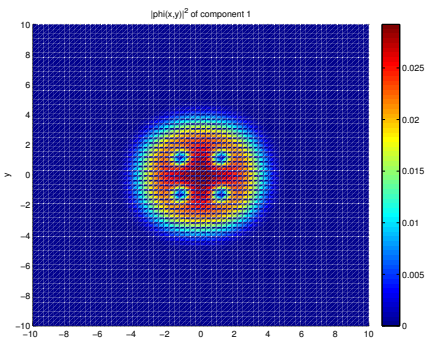

(c) BEFD: $J=2^{6}$

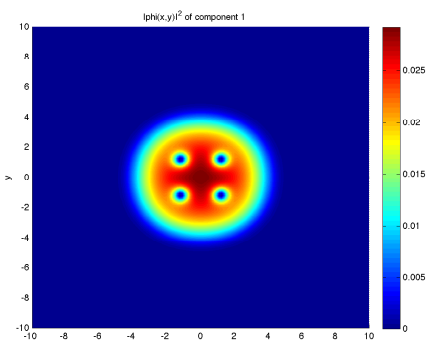

(e) BEFD: $J=2^{7}$

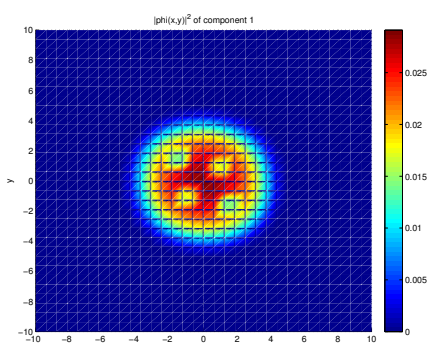

(b) BESP: $J=2^{5}$

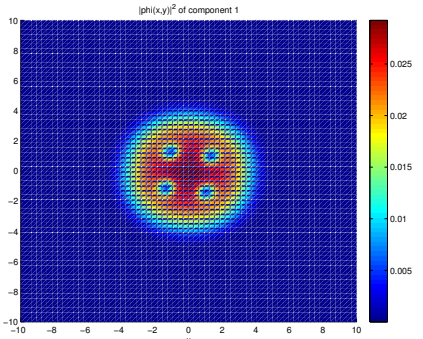

(d) BESP: $J=2^{6}$

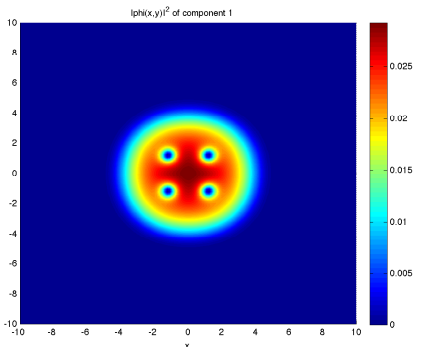

(f) BESP: $J=2^{7}$

Fig. 5 Representation of $\left|\phi_{J}\right|^{2}$ obtained by BEFD (left) and BESP (right) for different spatial discretizations.

\subsection{Which initial guess for CNGF?}

As we discussed above, our goal is to compute a (global) minimizer of the optimization problem 23). Before any numerical computation by BESP (or another iterative scheme), it is quite natural to prospect if some explicit exact or approximate solutions to the GPE are available. This is important for two reasons. Indeed, having such a solution allows to better understand the physical properties of the GPEs and BECs from the mathematical point of view. Many developments can be found in 
the Physics literature [97, 99]. We give below the example of the Thomas-Fermi approximation. In addition, since the optimization problem is extremely complex, it is unexpected to get an analytical solution for the problem, valid for any interesting situation (weak or strong nonlinearity, various potentials, inclusion of a rotation term,...). Since the optimization problem is nonlinear, there is no other alternative than developing some iterative numerical methods. To this end, we need to determine a suitable initial guess that is injected into the algorithm. In particular, a wellchosen approximate analytical solution can play this role. In Section 1.4 (page 17), we distinguished two cases where it is possible to build an approximate solution. Let us precise these approximations for different situations.

When there is no rotation (i.e. $\Omega=0$ ) and the potential is confining, the minimization problem (23) admits a unique global solution $\phi_{g}$ up to a phase factor [87]. For a potential $V$ such that: $\forall \mathbf{x} \in \mathbb{R}^{d}, V(\mathbf{x})=V_{0}(\mathbf{x})+W(\mathbf{x})$, where

$$
V_{0}(\mathbf{x})=\frac{1}{2} \sum_{j=1}^{d} \gamma_{x_{j}}^{2} x_{j}^{2} \text { and } \lim _{|\mathbf{x}| \rightarrow \infty} \frac{W(\mathbf{x})}{V(\mathbf{x})}=0,
$$

and for a weak nonlinear interaction (for example $|f(1)| \leq 10$ ), a suitable approximation [21] of the fundamental state of problem (21) is given by

$$
\forall \mathbf{x} \in \mathbb{R}^{d}, \quad \phi_{\mathrm{osc}}(\mathbf{x})=\frac{\left(\prod_{j=1}^{d} \gamma_{x_{j}}\right)^{1 / 4}}{\pi^{d / 4}} e^{-\frac{1}{2} \sum_{j=1}^{d} \gamma_{x_{j}} x_{j}^{2}},
$$

which corresponds to the fundamental state of the quantum harmonic oscillator [87]

$$
\left\{\begin{array}{l}
i \partial_{t} \psi(t, \mathbf{x})=-\frac{1}{2} \Delta \psi(t, \mathbf{x})+V_{0}(\mathbf{x}) \psi(t, \mathbf{x}), \forall t \in \mathbb{R}^{+}, \forall \mathbf{x} \in \mathbb{R}^{d}, \\
\psi(0, \mathbf{x})=\psi_{0}(\mathbf{x}) \in L_{\mathbf{x}}^{2} .
\end{array}\right.
$$

If one considers now a rotation term (i.e. $\Omega \neq 0$ ), finding a good approximation is much more problematic. In particular, the solution to the minimization problem is not necessarily unique, local minimizers possibly exist $(23)$ and there is sometimes not even existence of a solution if the rotation is too large [107]. In the case of a harmonic potential (59), the critical velocity above which there is no existence of a fundamental state is given by $\Omega_{c}=\min \left\{\gamma_{x}, \gamma_{y}\right\}$. In [101], the author shows that some phase transition phenomenae occur with respect to the rotation velocity when a quadratic-plus-quartic potential is considered. In particular, it is proved that a second critical velocity exists above which a giant vortex is created. For more details about the theory of quantum vortices, we refer to Rougerie \& al. [37, 49, 102], Aftalion \& al. [5, 6, 7, 8] and Tsubota \& al. [80, 81, 119]. An initial data allowing to converge towards the correct fundamental state has been proposed by Bao, Wang \& Markowich [32] and consists in choosing the following approximation

$$
\phi(\mathbf{x})=\frac{(1-\Omega) \phi_{\mathrm{osc}}(\mathbf{x})+\Omega \phi_{\mathrm{osc}}^{v}(\mathbf{x})}{\left\|(1-\Omega) \phi_{\mathrm{osc}}(\mathbf{x})+\Omega \phi_{\mathrm{osc}}^{v}(\mathbf{x})\right\|_{L_{\mathbf{x}}^{2}}},
$$


with

$$
\phi_{\mathrm{osc}}(\mathbf{x})=e^{-\frac{1}{2}\left(\gamma_{x} x^{2}+\gamma_{y} y^{2}\right)} \quad \text { and } \quad \phi_{\mathrm{osc}}^{v}(\mathbf{x})=\left(\gamma_{x} x+i \gamma_{y} y\right) e^{-\frac{1}{2}\left(\gamma_{x} x^{2}+\gamma_{y} y^{2}\right)} .
$$

This approximation is in fact an interpolation of the gaussian 60 and the same gaussian with an added centered vortex (singularity). In the case of a confining potential in the $x$-direction, we can simplify the equation as a two-dimensional GPE (see Section 1.5.2, page 21). Moreover, by using the polar coordinates and for an isotropic potential $\gamma_{x}=\gamma_{y}$, we obtain [32]

$$
\phi_{\mathrm{osc}}^{v}(\mathbf{x})=\frac{\gamma_{x}^{2} e^{i m \theta}}{\sqrt{\pi}} e^{-\gamma_{x} r^{2} / 2}
$$

where $m=1$ is the "winding number" of the central vortex and corresponds to the first vortex mode. By using these initial data, it is possible to converge to the fundamental state in the case of a subcritical velocity $\Omega<\Omega_{c}$. We present on Figure 6(a) 6(b) the initial data 62 in $2 d$ for two rotation speeds. For completeness, we report the three- and one-dimensional cases (respectively, on Figures 7 (for two rotation velocities) and 8 .

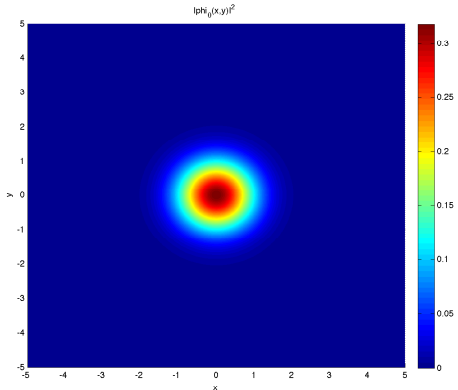

(a) $\Omega=0$

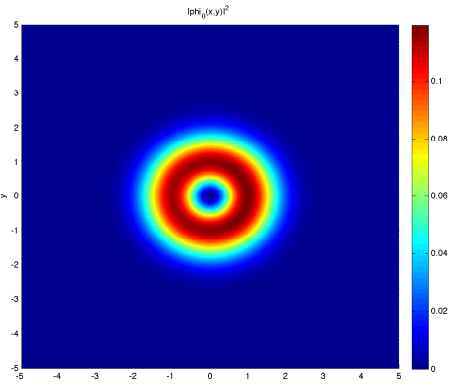

(b) $\Omega=0.99$

Fig. 6 Representation of $\left|\phi_{0}\right|^{2}$ for the two-dimensional harmonic potential problem $\left(\gamma_{x}=\gamma_{y}=1\right)$ with a weak nonlinear interaction, without and with a rotation term, by using formula 62 .

In the case of a strong interaction, we consider the Thomas-Fermi approximation (cf. Section 1.4, page 17) which consists in neglecting the kinetic energy related to the Laplacian and rotation operators. One then gets a simplified minimization problem where the energy is given by

$$
\mathscr{E}_{\Omega, F}(\psi) \approx \mathscr{E}_{\mathrm{TF}}(\psi):=\int_{\mathbb{R}^{d}}\left[V(\mathbf{x})|\psi(t, \mathbf{x})|^{2}+F\left(|\psi(t, \mathbf{x})|^{2}\right)\right] d \mathbf{x} .
$$

More precisely, coming back to an eigenvalue problem similarly to the general case 24, we are looking for the eigenfunction $\phi_{\mathrm{TF}} \in L_{\mathbf{x}}^{2}$ and the eigenvalue $\mu_{\mathrm{TF}} \in \mathbb{R}$ of 


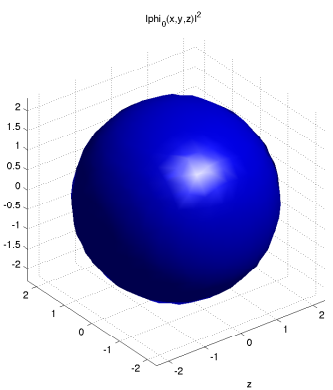

(a) $\Omega=0$

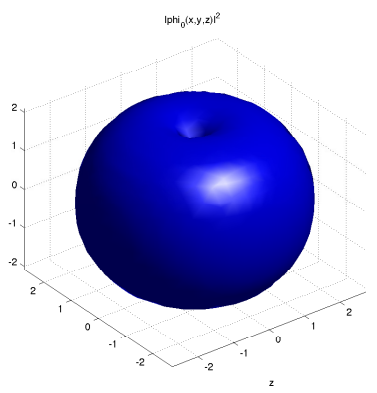

(b) $\Omega=0.99$

Fig. 7 Isovalues $\left|\phi_{0}\right|^{2}=10^{-3}$ for a three-dimensional harmonic problem $\left(\gamma_{x}=\gamma_{y}=\gamma_{z}=1\right)$ with a weak interaction, without and with a rotation term, by using formula 62.

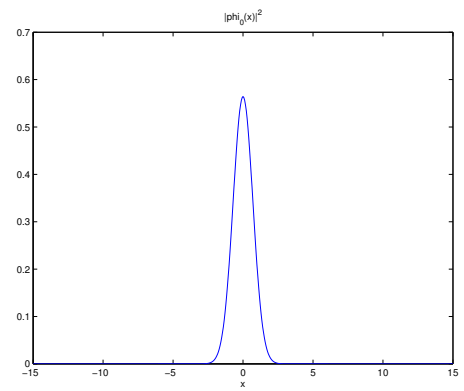

Fig. 8 Representation of $\left|\phi_{0}\right|^{2}$ for a one-dimensional harmonic problem $\left(\gamma_{x}=1\right)$ with a weak interaction.

the problem

$$
\mu_{\mathrm{TF}} \phi_{\mathrm{TF}}=f\left(\left|\phi_{\mathrm{TF}}\right|^{2}\right) \phi_{\mathrm{TF}}+V \phi_{\mathrm{TF}},
$$

under the normalization constraint $\mathscr{N}\left(\phi_{\mathrm{TF}}\right)=\left\|\phi_{\mathrm{TF}}\right\|_{L_{\mathbf{x}}^{2}}=1$. We obtain

$$
\forall \mathbf{x} \in \operatorname{supp}\left(\phi_{\mathrm{TF}}\right), \quad \mu_{\mathrm{TF}}=f\left(\left|\phi_{\mathrm{TF}}\right|^{2}\right)+V(\mathbf{x}) .
$$

By assuming that it is possible to inverse the function $f$ on $\mathbb{R}$, we can then deduce an explicit form of $\phi_{\mathrm{TF}}$ which is assumed to be real-valued,

$$
\forall \mathbf{x} \in \mathbb{R}^{d}, \quad \phi_{\mathrm{TF}}(\mathbf{x})= \begin{cases}\sqrt{f^{-1}\left(\mu_{\mathrm{TF}}-V(\mathbf{x})\right)}, & \text { for } f^{-1}\left(\mu_{\mathrm{TF}}-V(\mathbf{x})\right)>0, \\ 0 \quad & \text { for } f^{-1}\left(\mu_{\mathrm{TF}}-V(\mathbf{x})\right) \leq 0 .\end{cases}
$$

To get $\mu$, we use the mass conservation. For a cubic nonlinearity $f\left(|\phi|^{2}\right)=\beta|\phi|^{2}$, with $\beta \in \mathbb{R}^{+}$, we can choose the following approximation of the fundamental state 


$$
\phi_{\mathrm{TF}}(\mathbf{x})= \begin{cases}\sqrt{\frac{\mu_{\mathrm{TF}}-V(\mathbf{x})}{\beta}}, & \text { for } \mu_{\mathrm{TF}}-V(\mathbf{x})>0, \\ 0 & , \text { for } \mu_{\mathrm{TF}}-V(\mathbf{x}) \leq 0,\end{cases}
$$

where $\mu_{\mathrm{TF}}$ is given by the expression [21]

$$
\mu_{\mathrm{TF}}=\frac{1}{2} \begin{cases}\left(3 \beta \gamma_{x}\right)^{2 / 3} & \text { for } d=1 \\ \left(4 \beta \gamma_{x} \gamma_{y}\right)^{1 / 2} & \text { for } d=2 \\ \left(\frac{15 \beta \gamma_{x} \gamma_{y} \gamma_{z}}{4 \pi}\right)^{2 / 5} & \text { for } d=3\end{cases}
$$

We represent on Figure 9 the moduli of the Thomas-Fermi approximations 66 for a quadratic potential $\left(\gamma_{x}=\gamma_{y}=\gamma_{z}=1\right)$ and a cubic nonlinearity in $1 \mathrm{~d}, 2 \mathrm{~d}$ and $3 \mathrm{~d}$.

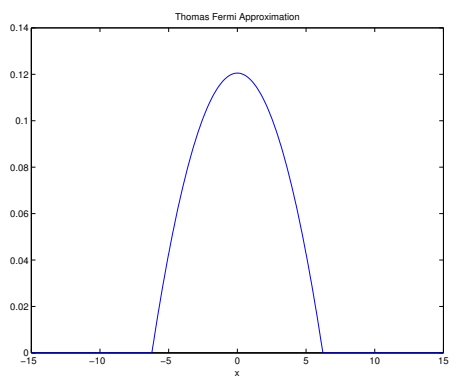

(a) Dimension $d=1$

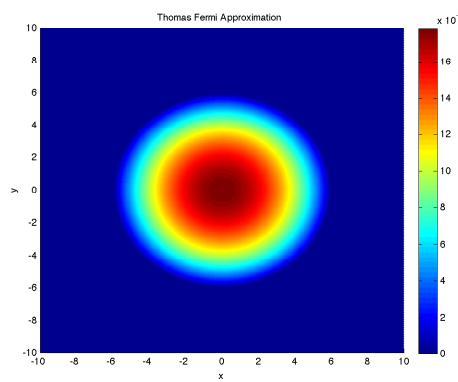

(b) Dimension $d=2$

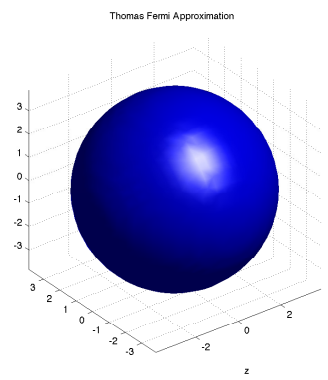

(c) Dimension $d=3$

Fig. 9 Representation of $\left|\phi_{0}\right|^{2}$ for the Thomas-Fermi approximation and a quadratic potential, $\beta=1000$ (strong interaction) for the $1 \mathrm{~d}, 2 \mathrm{~d}$ and $3 \mathrm{~d}$ cases.

The Thomas-Fermi approximation has the advantage of being less restrictive than the weak interaction approximation concerning the classes of potentials and nonlinearities that are eligible. In particular, the following potentials can be considered $(d=2)$

- Quadratic-plus-quartic potential [124] 


$$
V(\mathbf{x})=(1-\alpha) \frac{1}{2}\left(\gamma_{x}^{2} x^{2}+\gamma_{y}^{2} y^{2}\right)+\frac{\kappa}{4}\left(\gamma_{x}^{2} x^{2}+\gamma_{y}^{2} y^{2}\right)^{2} .
$$

- Quadratic-plus-gaussian potential [77]

$$
V(\mathbf{x})=\frac{1}{2}\left(\gamma_{x}^{2} x^{2}+\gamma_{y}^{2} y^{2}\right)+w_{0} e^{-\frac{\left(x-x_{0}\right)^{2}+\left(y-y_{0}\right)^{2}}{d^{2}}} .
$$

- Quadratic-plus-sine potential (also called optical potential) [124]

$$
V(\mathbf{x})=\frac{1}{2}\left(\gamma_{x}^{2} x^{2}+\gamma_{y}^{2} y^{2}\right)+\frac{a_{1}}{2} \sin \left(\frac{\pi x}{d_{1}}\right)^{2}+\frac{a_{2}}{2} \sin \left(\frac{\pi y}{d_{2}}\right)^{2} .
$$

- Double-well potential [122]

$$
V(\mathbf{x})=\frac{1}{2}\left(\gamma_{x}^{2} x^{2}+\gamma_{y}^{2} y^{2}\right)+V_{0} e^{-x^{2} / 2 d^{2}} .
$$

Examples of Thomas-Fermi approximations for these potentials are given on Figures 10 and 11

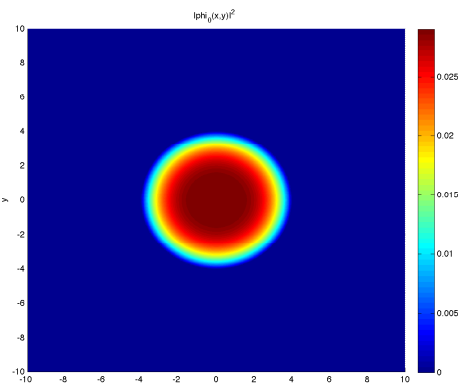

(a) $\gamma_{x}=\gamma_{y}=1$

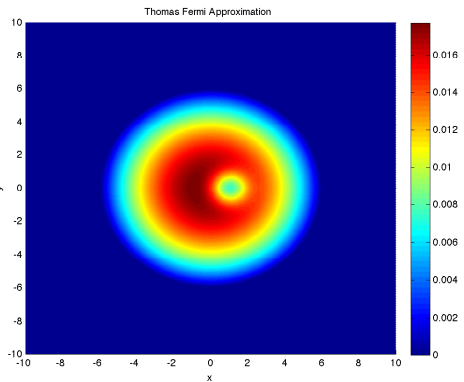

(b) $\gamma_{x}=\gamma_{y}=1 ; \mathbf{x}_{0}=(1,0) ; d=1 ; w_{0}=10$

Fig. 10 Examples of Thomas-Fermi approximations for potentials 68 (left) and 69 (right).

\subsection{Solving BESP linear systems: the fixed point method, its limitations and Krylov subspace iterative solvers}

We consider now the BESP scheme 


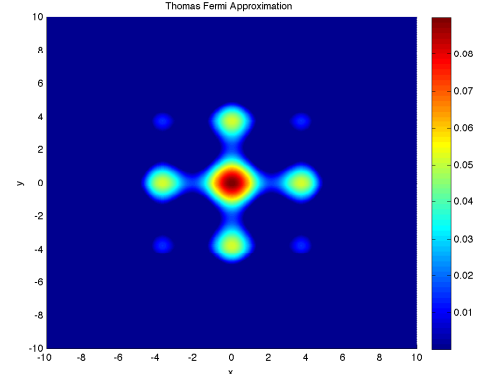

(a) $\gamma_{x}=\gamma_{y}=1 ; a_{1}=a_{2}=25 ; d_{1}=d_{2}=4$

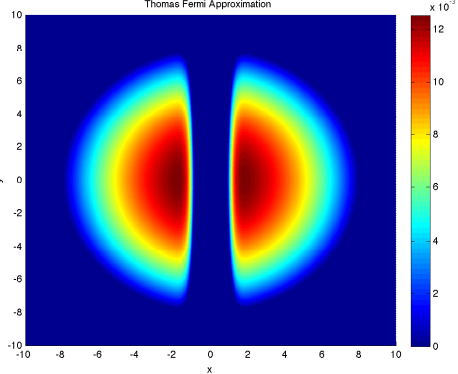

(b) $\gamma_{x}=\gamma_{y}=1 / 2 ; V_{0}=40 ; d=0.5$

Fig. 11 Examples of Thomas-Fermi approximations for potentials 70 (left) and 71 (right).

$$
\left\{\begin{array}{l}
\mathbb{A}^{\mathrm{BE}, n} \tilde{\phi}=\mathbf{b}^{\mathrm{BE}, n} \\
\phi^{n+1}=\frac{\tilde{\phi}}{\|\tilde{\phi}\|_{\ell_{\pi}^{2}}} \\
\phi^{0}:=\phi_{0}
\end{array}\right.
$$

where, for the sake of conciseness, we set: $\mathbb{A}^{\mathrm{BE}, n}:=\mathbb{A}_{\mathrm{SP}}^{\mathrm{BE}, n}$. At each iteration $n$, it is clear that the minimization method requires the solution of a linear system: $\mathbb{A}^{\mathrm{BE}, n} \tilde{\phi}=\mathbf{b}^{\mathrm{BE}, n}$. Since we use pseudo-spectral approximation methods, the operator $\mathbb{A}^{\mathrm{BE}, n}$ is given implicitly through a FFT, meaning that the matrix $\mathbb{A}^{\mathrm{BE}, n}$ is not explicitly known by its coefficients. As a consequence, using a direct matrix solver is not permitted. An alternative solution consists in considering a matrix-free iterative method. A first approach, introduced by Bao et al. [21] for non rotating GPEs, is based on stationary (fixed-point) methods. It has been next extended to rotating BEC by Zeng \& Zhang [124]. Nevertheless, in [12], some examples show that the method does not converge when the rotation speed $\Omega$ is too large. In [12], the introduction of Krylov subspace iterative solvers (GMRES, BiCGStab) accelerated by simple operator-based preconditioners provides robust and fast iterative methods that can be easily extended to the mutli-components case.

\subsection{Extension to multi-components BECs}

In this Section, we present the extension of BESP to BECs with $N_{c} \in \mathbb{N}$ components. The GPEs system that describes this situation is

$$
\left\{\begin{aligned}
i \partial_{t} \Psi(t, \mathbf{x})=-\frac{1}{2} \Delta \Psi(t, \mathbf{x})- & \Omega L_{z} \Psi(t, \mathbf{x})+\mathbf{V}(\mathbf{x}) \Psi(t, \mathbf{x}) \\
& +\mathbf{f}(\Psi) \Psi(t, \mathbf{x}), \forall t \in \mathbb{R}^{+}, \forall \mathbf{x} \in \mathbb{R}^{d} \\
\Psi(0, \mathbf{x})=\Psi_{0}(\mathbf{x}) \in L_{\mathbf{x}}^{2, N_{c}}, \forall \mathbf{x} & \in \mathbb{R}^{d}
\end{aligned}\right.
$$


where we set $\Psi(t, \mathbf{x})=\left(\Psi_{\ell}(t, \mathbf{x})\right)_{\ell \in\left\{1, \ldots, N_{c}\right\}}$ and $|\Psi(t, \mathbf{x})|^{2}=\sum_{\ell=1}^{N_{c}}\left|\Psi_{\ell}(t, \mathbf{x})\right|^{2}$. The non diagonal operators involved in this system are: $\mathbf{V}(\mathbf{x})=\left(\mathbf{V}_{\ell, m}(\mathbf{x})\right)_{\ell, m \in\left\{1, \ldots, N_{c}\right\}}$, and

$$
\mathbf{f}(\Psi)=\left(\mathbf{f}_{\ell, m}\left(\Psi_{1}, \ldots, \Psi_{N_{c}}, \Psi_{1}^{*}, \ldots, \Psi_{N_{c}}^{*}\right)\right)_{\ell, m \in\left\{1, \ldots, N_{c}\right\}} .
$$

For the partial differential operators, we have

$$
\Delta \Psi(t, \mathbf{x})=\left(\Delta \Psi_{\ell}(t, \mathbf{x})\right)_{\ell \in\left\{1, \ldots, N_{c}\right\}}, \quad L_{z} \Psi(t, \mathbf{x})=\left(L_{z} \Psi_{\ell}(t, \mathbf{x})\right)_{\ell \in\left\{1, \ldots, N_{c}\right\}} .
$$

We furthermore assume that $\mathbf{f}_{\ell, m}, 1 \leq \ell, m \leq N_{c}$, are smooth real-valued polynomial functions and that the operators $\mathbf{V}$ and $\mathbf{f}$ are symmetrical, i.e. $\mathbf{V}_{\ell, m}=\mathbf{V}_{m, \ell}$ and $\mathbf{f}_{\ell, m}=$ $\mathbf{f}_{m, \ell}, 1 \leq \ell, m \leq N_{c}$, in such a way that we have the mass conservation. For the multicomponents case, let us recall that the mass is given by

$$
\mathscr{N}(\Psi):=\|\Psi\|_{L_{\mathbf{x}}^{2}}^{2}=\sum_{\ell=1}^{N_{c}}\left\|\Psi_{\ell}(t, \mathbf{x})\right\|_{L_{\mathbf{x}}^{2}}^{2}=\sum_{\ell=1}^{N_{c}} \int_{\mathbb{R}^{d}}\left|\Psi_{\ell}(t, \mathbf{x})\right|^{2} d \mathbf{x},
$$

and the energy by

$$
\begin{aligned}
\mathscr{E}_{\Omega, \mathbf{F}}(\Psi):=\sum_{\ell=1}^{N_{c}} \int_{\mathbb{R}^{d}}\left(\frac{1}{2}\left|\nabla \Psi_{\ell}(t, \mathbf{x})\right|^{2}-\Omega \Psi_{\ell}^{*}(t, \mathbf{x}) L_{z} \Psi_{\ell}(t, \mathbf{x})\right) d \mathbf{x} \\
+\sum_{\ell, m=1}^{N_{c}} \int_{\mathbb{R}^{d}} \mathbf{V}_{\ell, m}(t, \mathbf{x}) \Psi_{\ell}^{*}(t, \mathbf{x}) \Psi_{m}(t, \mathbf{x})+\mathbf{F}_{\ell, m}(\Psi) d \mathbf{x},
\end{aligned}
$$

where

$$
\mathbf{F}_{\ell, m}(\Psi):=\int_{0}^{1} \mathbf{f}_{\ell, m}\left(\Psi_{1}, \ldots, \Psi_{N_{c}}, \Psi_{1}^{*}, \ldots, \imath \Psi_{\ell}^{*}, \ldots, \Psi_{N_{c}}^{*}\right) \Psi_{\ell}^{*} \Psi_{m} d \imath
$$

\subsubsection{CNGF for multi-components BECs}

Similarly to the proof detailed in Section 1.4 (page 17), we show that a stationary state is a critical point of the energy functional, i.e. it is solution to the minimization problem: find a function $\Phi \in L_{\mathbf{x}}^{2, N_{C}}$ such that

$$
\mathscr{E}_{\Omega, \mathbf{F}}(\Phi)=\min _{\mathscr{N}(\Psi)=1} \mathscr{E}_{\Omega, \mathbf{F}}(\Psi)
$$

The CNGF method directly applies to the multi-components case 


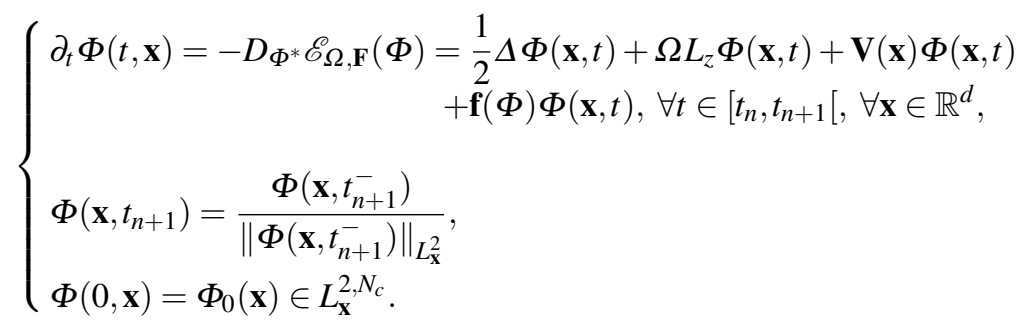

This problem has been studied for example in [16, 17]. Let us recall the following result which proves that the energy associated with the solution to 75 is decaying under suitable assumptions.

Theorem 2 Let us assume that the potential operator is diagonal, i.e. $\mathbf{V}(\mathbf{x})=$ $\left(\mathbf{V}_{\ell}(\mathbf{x})\right)_{\ell \in\left\{1, \ldots, N_{c}\right\}}$, and is such that $\mathbf{V}_{\ell}(\mathbf{x}) \geq 0, \forall \mathbf{x} \in \mathbb{R}^{d}, \forall \ell \in\left\{1, \ldots, N_{c}\right\}$. Furthermore, we suppose that the nonlinearity is diagonal: $\mathbf{f}(\Phi)=\left(\mathbf{f}_{\ell}(\Psi)\right)_{\ell \in\left\{1, \ldots, N_{c}\right\}}$, and such that $\mathbf{f}_{\ell}(\Psi)=\sum_{m=1}^{N_{c}} \beta_{\ell, m}\left|\phi_{m}\right|^{2}\left|\phi_{\ell}\right|^{2}$, with $\beta_{\ell, m} \geq 0, \forall \ell, m \in\left\{1, \ldots, N_{c}\right\}$. Finally, we consider that there is no rotation, i.e. $\Omega=0$. Then, the solution $\Phi$ to (75) satisfies, $\forall n \in \mathbb{N}$,

$$
\forall t \in\left[t_{n}, t_{n+1}\left[, \quad \mathscr{E}_{0, \mathbf{F}}(\Phi(\mathbf{x}, t)) \leq \mathscr{E}_{0, \mathbf{F}}\left(\Phi\left(\mathbf{x}, t_{n}\right)\right) .\right.\right.
$$

\subsubsection{BESP for multi-components BECs}

We now essentially focus on the semi-implicit backward Euler time discretization of 75 ,

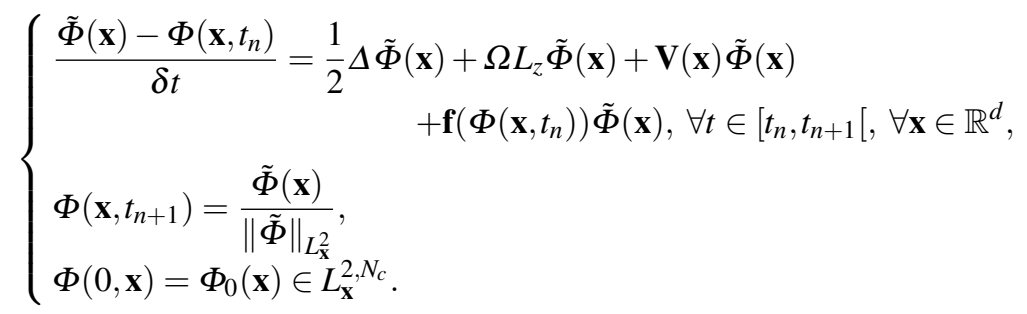

Let us precise the spatial discretization of 76 leading to BESP. We consider that $d=2$, the extension to $d=1$ and $d=3$ being straightforward. The computational box is $\mathscr{O}:=]-a_{x}, a_{x}[\times]-a_{y}, a_{y}\left[\right.$. The associated discrete grid $\mathscr{O}_{J, K}$ is given by (30). Let: $\mathscr{P}_{N_{c}, J, K}=\left\{(\ell, j, k) \in \mathbb{N}^{3} ; 1 \leq \ell \leq N_{c}, 1 \leq j \leq J\right.$ and $\left.1 \leq k \leq K\right\}$. For the pseudo-spectral approximation, the multi-components Laplacian is discretized by

$$
\forall(\ell, j, k) \in \mathscr{P}_{N_{c}, J, K}, \quad([[\Delta]] \Phi)_{\ell, j, k}=\left([[\Delta]] \Phi_{\ell}\right)_{(j, k)},
$$

where $[[\Delta]]$ that appears in the right-hand side is given by the expression 43 . Similarly, the multi-components rotation operator is discretized by

$$
\forall(\ell, j, k) \in \mathscr{P}_{N_{c}, J, K}, \quad\left(\left[\left[L_{z}\right]\right] \Phi\right)_{\ell, j, k}=\left(\left[\left[L_{z}\right]\right] \Phi_{\ell}\right)_{(j, k)},
$$


where $\left[\left[L_{z}\right]\right]$ is fixed by $[42$. For the potential and nonlinear operators, the discretization is direct on the grid $\mathscr{O}_{J, K}$

$$
[[\mathbf{V}]]:=\left(\begin{array}{cccc}
{\left[\left[\mathbf{V}_{1,1}\right]\right]} & {\left[\left[\mathbf{V}_{1,2}\right]\right]} & \cdots & {\left[\left[\mathbf{V}_{1, N_{c}}\right]\right]} \\
{\left[\left[\mathbf{V}_{2,1}\right]\right]} & {\left[\left[\mathbf{V}_{2,2}\right]\right]} & \cdots & {\left[\left[\mathbf{V}_{2, N_{c}}\right]\right]} \\
\vdots & \vdots & \ddots & \vdots \\
{\left[\left[\mathbf{V}_{N_{c}, 1}\right]\right]} & {\left[\left[\mathbf{V}_{2, N_{c}}\right]\right]} & \cdots & {\left[\mathbf{V}_{N_{c}, N_{c}}\right]}
\end{array}\right) \in \mathscr{M}_{M N_{c}}(\mathbb{C}),
$$

where $\left[\left[\mathbf{V}_{m, \ell}\right]\right]$ is given by $[44$, and

$$
\left[\left[\mathbf{f}\left(\Phi^{n}\right)\right]\right]:=\left(\begin{array}{cccc}
{\left[\left[\mathbf{f}_{1,1}\left(\Phi^{n}\right)\right]\right]} & \left.\left[\mathbf{f}_{1,2}\left(\Phi^{n}\right)\right]\right] & \cdots & \left.\left[\mathbf{f}_{1, N_{c}}\left(\Phi^{n}\right)\right]\right] \\
{\left[\left[\mathbf{f}_{2,1}\left(\Phi^{n}\right)\right]\right]} & {\left[\left[\mathbf{f}_{2,2}\left(\Phi^{n}\right)\right]\right]} & \cdots & {\left[\left[\mathbf{f}_{2, N_{c}}\left(\Phi^{n}\right)\right]\right]} \\
\vdots & \vdots & \ddots & \vdots \\
{\left[\left[\mathbf{f}_{N_{c}, 1}\left(\Phi^{n}\right)\right]\right]} & \left.\left[\mathbf{f}_{2, N_{c}}\left(\Phi^{n}\right)\right]\right] & \cdots & \left.\left[\mathbf{f}_{N_{c}, N_{c}}\left(\Phi^{n}\right)\right]\right]
\end{array}\right) \in \mathscr{M}_{M N_{c}}(\mathbb{C})
$$

where $\left[\left[\mathbf{f}_{m, \ell}\left(\Phi^{n}\right)\right]\right], \forall \ell, m \in\left\{1, \ldots, N_{c}\right\}$, is defined, for any vector field $\varphi \in \mathbb{C}^{M}$, $\forall(j, k) \in \mathscr{P}_{J, K}$, by

$$
\left(\left[\mathbf{f}_{m, \ell}\left(\Phi^{n}\right)\right] \varphi\right)_{j, k}=\mathbf{f}_{m, \ell}\left(\Phi^{n}\left(\mathbf{x}_{j, k}\right)\right) \varphi_{j, k},
$$

with $\Phi^{n}(\mathbf{x})=\Phi\left(\mathbf{x}, t_{n}\right)$ for 76 . Setting $\tilde{\Phi} \in \mathbb{C}^{M N_{c}}$ as the solution to 76 , one obtains the BESP scheme, $\forall n \in \mathbb{N}$,

$$
\left\{\begin{array}{l}
\mathbb{A}^{\mathrm{BE}, n} \tilde{\Phi}=\mathbf{b}^{\mathrm{BE}, n}, \\
\Phi^{n+1}=\frac{\tilde{\Phi}}{\|\tilde{\Phi}\|_{\ell_{\pi}^{2}}},
\end{array}\right.
$$

where the operator $\mathbb{A}^{\mathrm{BE}, n}$ maps a given vector $\Phi \in \mathbb{C}^{M N_{c}}$ to $\Psi \in \mathbb{C}^{M N_{c}}$ through

$$
\begin{aligned}
& \Psi:=\mathbb{A}^{\mathrm{BE}, n} \Phi=\mathbb{A}_{\mathrm{TF}}^{\mathrm{BE}, n} \Phi+\mathbb{A}_{\Delta, \Omega}^{\mathrm{BE}, n} \Phi, \\
& \mathbb{A}_{\mathrm{TF}, n}^{\mathrm{BE}} \Phi:=\left(\frac{[[\mathbf{I}]]}{\delta t}+[[\mathbf{V}]]+\left[\left[\mathbf{f}\left(\Phi^{n}\right)\right]\right]\right) \Phi, \\
& \mathbb{A}_{\Delta, \Omega}^{\mathrm{BE}} \Phi:=\left(-\frac{1}{2}\left[[[]]-\Omega\left[\left[L_{z}\right]\right]\right) \Phi .\right.
\end{aligned}
$$

The right-hand side is

$$
\mathbf{b}^{\mathrm{BE}, n}:=\frac{\Phi^{n}}{\delta t}
$$

The matrix $[[\mathbf{I}]]$ is the identity matrix of $\mathscr{M}_{M N_{c}}(\mathbb{C})$. Finally, the discrete $L_{\mathbf{x}}^{2, N_{c}}$-norm of a vector $\Phi \in \mathbb{C}^{M N_{c}}$ is defined by

$$
\|\Phi\|_{\ell_{\pi}^{2}}:=\left(\sum_{\ell=1}^{N_{c}}\left\|\Phi_{\ell}\right\|_{\ell_{\pi}^{2}}^{2}\right)^{1 / 2}
$$

Furthermore, we define the discrete (strong) stopping criterion as 


$$
\left\|\Phi^{n+1}-\Phi^{n}\right\|_{\infty}<\varepsilon \delta t
$$

with the discrete uniform norm defined by: $\forall \Phi \in \mathbb{C}^{M N_{c}},\|\Phi\|_{\infty}=\sum_{\ell=1}^{N_{c}} \max _{(j, k) \in \mathscr{P}_{J, K}}\left|\Phi_{\ell, j, k}\right|$, and the discrete (weak) stopping criterion as

$$
\left|\mathscr{E}_{\Omega, \mathbf{F}}\left(\Phi^{n+1}\right)-\mathscr{E}_{\Omega, \mathbf{F}}\left(\Phi^{n}\right)\right|<\varepsilon \delta t,
$$

with the discrete energy

$$
\begin{aligned}
& \mathscr{E}_{\Omega, \mathbf{F}}(\Phi)= \\
& \left(h_{x} h_{y}\right)^{1 / 2} \sum_{\substack{(j, k) \in \mathscr{P}_{J, K} \\
1 \leq \ell \leq N_{C}}} \operatorname{Re}\left\{\Phi_{\ell, j, k}^{*}\left(-\frac{1}{2}[[\Delta]] \Phi-\Omega\left[\left[L_{z}\right]\right] \Phi+[[V]] \Phi+\left[\left[F\left(|\Phi|^{2}\right)\right]\right] \Phi\right)_{\ell, j, k}\right\} .
\end{aligned}
$$

As in the one-component case, preconditioned Krylov subspace solvers can be used to iteratively solve the associated linear systems (see [12]).

\section{Computation: the Gross-Pitaevskii Equation Laboratory}

\subsection{GPELab: a short presentation}

As seen in Section 2 for the stationary state computation and as it will be explained in Section 4 for the dynamics, the numerical methods that we present are robust and efficient. Furthermore, they can be quite directly extended to different kinds of Gross-Pitaevskii Equations and systems. The aim of this Section is to present a freely available Matlab toolbox called GPELab ${ }^{1}$ (Gross-Pitaevskii Equation Laboratory) which is based on these advanced numerical schemes. The computational tools are developed in such a way that they can be easily used by physicists working on BECs. GPELab allows the user to make various computations in $1 \mathrm{~d}-2 \mathrm{~d}-3 \mathrm{~d}$, for multi-components GPEs with general potentials and nonlinearities. In addition, the stochastic effects that are described for the dynamics can be numerically simulated according to efficient and accurate schemes. Even if GPELab is dedicated to Gross-Pitaevskii Equations, it is more generally useful when one wants to solve problems related to nonlinear Schrödinger equations. Let us remark that at the time of writing this contribution, other interesting computational codes for solving GPEs (with a cubic nonlinearity) are proposed by different authors. In [118], a Fortran 90 solver based on the imaginary time method can solve the stationary state problem for the one-component GPE with a quadratic potential and without rotation term. In [95, 120], the authors distribute finite difference Fortran 90 codes for one-component problems with radial and spherical potentials, and no rotation. Improvements, in particular the parallelization of the code with OpenMP, are provided

1 http://gpelab.math.cnrs.fr/ 
in [120]. Other codes (developed with Fortran or Matlab) for GPEs are available [44, 75, 90]. Nevertheless, it seems that none of these solvers propose the flexibility that GPELab offers where many physical situations of interest can be considered: any potential and nonlinearity, inclusion of gradient-like terms for fast rotations, multi-components cases, stationary states and dynamics of BECs, stochastic effects. To show how GEPLab is powerful, we now consider a few numerical examples. Other interesting situations (with downloadable source files) are given in the GPELab user guide and the associated papers [11, 13].

\subsection{Experiment I: stationary state of a $1 \mathrm{~d}$ BEC with Josephson junction}

In this example, we want to reproduce the numerical simulations obtained in [17] where the following one-dimensional $(d=1)$ system of GPEs with a Josephson junction is considered

$$
\left\{\begin{array}{l}
i \partial_{t} \psi_{1}=\left[-\frac{1}{2} \Delta+V(\mathbf{x})+\delta+\left(\beta_{11}\left|\psi_{1}\right|^{2}+\beta_{12}\left|\psi_{2}\right|^{2}\right)\right] \psi_{1}+\lambda \psi_{2}, \\
i \partial_{t} \psi_{2}=\left[-\frac{1}{2} \Delta+V(\mathbf{x})+\left(\beta_{22}\left|\psi_{2}\right|^{2}+\beta_{12}\left|\psi_{1}\right|^{2}\right)\right] \psi_{2}+\lambda \psi_{1} .
\end{array}\right.
$$

In the above system, $\delta$ is the detuning constant of the Raman transition, $\beta_{j k}$ are the interaction constants between the gazes and $\lambda$ is the effective Rabi frequency. We use BESP for a time step $\delta t=10^{-1}$ and a uniform spatial grid with $2^{10}+1$ points on ] $-16,16$ [. In addition, the (strong) stopping criterion for computing the stationary states is $10^{-6}$. Following [17], the values of the physical parameters are: $\lambda=-1, \delta=0, \beta=500, \beta_{11}=\beta, \beta_{12}=0.94 \beta, \beta_{22}=0.97 \beta$. The initial data is a centered gaussian for each component. At the end of the computation, we obtain each component of the stationary state and some interesting physical outputs (see Table 3). We can also simultaneously print out the moduli of the components and conclude that they are the same as the ones reported in [17] (see Figure 12].

\subsection{Experiment II: stationary state of a fast rotating $2 d$ BEC in a strongly confining trap}

We consider the stationary state computation for a two-dimensional $(d=2)$ GPE with a quadratic-plus-quartic potential, a cubic nonlinearity and a rotation operator

$$
\begin{gathered}
i \partial_{t} \psi=\frac{1}{2} \Delta \psi+\left[\frac{1-\alpha}{2}\left(\gamma_{x}|x|^{2}+\gamma_{y}|y|^{2}\right)+\frac{\kappa}{4}\left(\gamma_{x}|x|^{2}+\gamma_{y}|y|^{2}\right)^{2}\right] \psi \\
+\beta|\psi|^{2} \psi+i \Omega\left(y \partial_{x}-x \partial_{y}\right) \psi,
\end{gathered}
$$




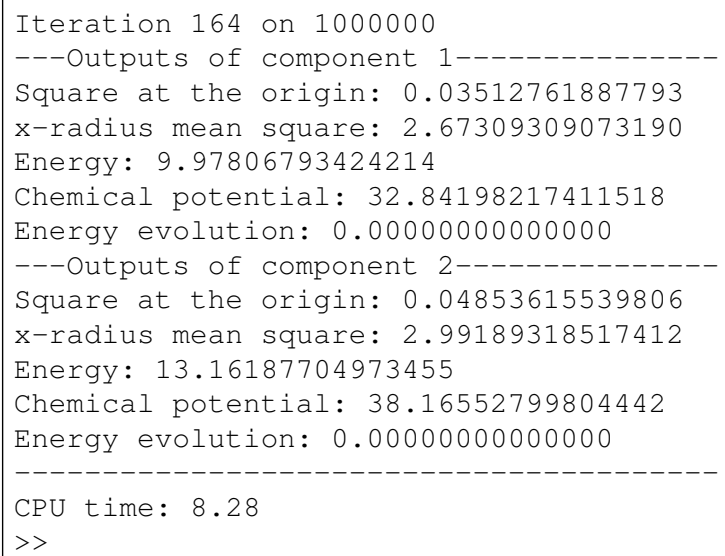

Table 3 Stationary states outputs for the two-components GPEs system with Josephson junction.

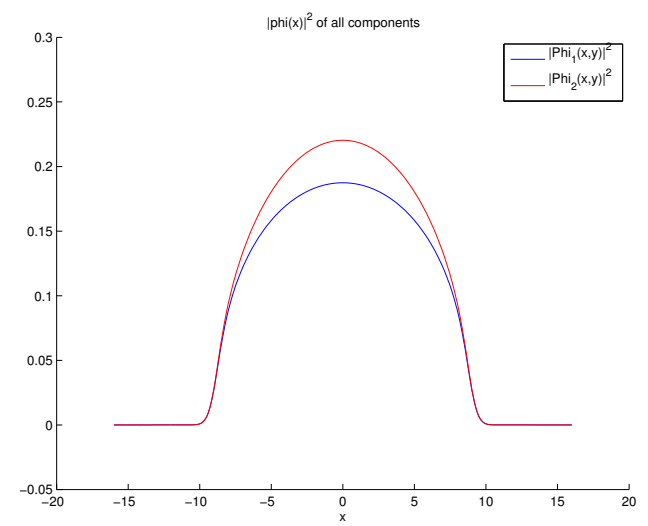

Fig. 12 Moduli of the two-components BEC.

with the parameters values $\alpha=1.2, \kappa=0.3, \gamma_{x}=\gamma_{y}=1, \beta=1000$ and $\Omega=3.5$. This is a typical example of a fast rotating BEC. We consider BESP with $\delta t=10^{-3}$. The computational domain is $]-10,10\left[^{2}\right.$, discretized by a uniform grid with $2^{8}+1$ points in each direction $x$ and $y$. The (strong) stopping criterion of BESP is $10^{-5}$ and the initial data is the Thomas-Fermi approximation associated with the physical problem. In Table 4, we report the outputs at the end of the simulation. We represent the modulus of the ground state on Figure 13 obtained by GPELab. In particular, we can see the existence of many uniformly distributed vortices in the annulus. 


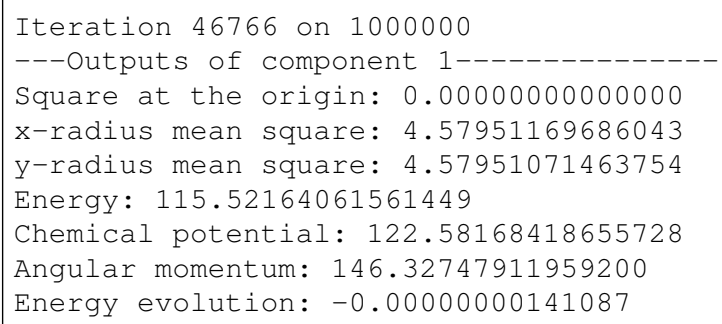

Table 4 Outputs at the end of the computation.

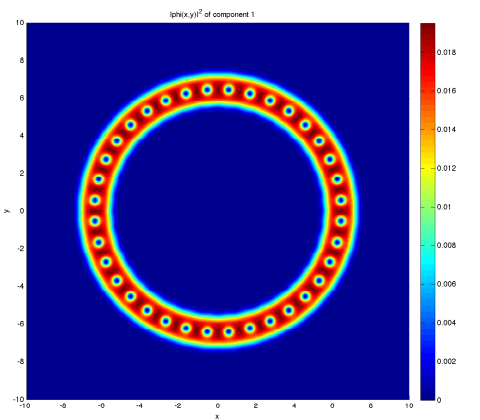

Fig. 13 Modulus of the converged stationary state.

\subsection{Experiment III: stationary state of a $3 d$ dipole-dipole BEC}

We show here a last numerical experiment for the three-dimensional $(d=3)$ GPE with a quadratic potential, a cubic nonlinearity to which a dipole-dipole nonlocal nonlinear interaction is added

$$
\begin{aligned}
i \partial_{t} \psi=\frac{1}{2} \Delta \psi & +\frac{1}{2}\left(\gamma_{x}|x|^{2}+\gamma_{y}|y|^{2}+\gamma_{z}|z|^{2}\right) \Psi \\
& +\beta|\psi|^{2} \psi+d^{2}\left(\int_{\mathbb{R}^{3}} \frac{1-3 \cos ^{2}(\widehat{\mathbf{a}, \tilde{\mathbf{x}}})}{\|(x, y, z)-\tilde{\mathbf{x}}\|^{3}}|\psi(t, \tilde{\mathbf{x}})|^{2} d \tilde{\mathbf{x}}\right) \psi
\end{aligned}
$$

with $\gamma_{x}=\gamma_{y}=\gamma_{z}=1, \beta=2000$ and $\mathbf{a}=(0,0,1)$. The discretization for BESP uses $\delta t=10^{-2}$ and a uniform grid with $2^{6}+1$ points in each direction $x, y$ and $z$ for the computational domain $]-15,15\left[^{3}\right.$. The (strong) stopping criterion is fixed to $10^{-6}$. In GEPLab, the nonlinearity which is defined by the dipole-dipole interaction can be efficiently computed by using FFTs via 


$$
\begin{aligned}
& d^{2} \int_{\mathbb{R}^{3}} \frac{1-3 \cos ^{2}(\widehat{\mathbf{a}, \tilde{\mathbf{x}}})}{\| \mathbf{x}-\tilde{\mathbf{x}}||^{3}} \mid\left.\psi(t, \tilde{\mathbf{x}})\right|^{2} d \tilde{\mathbf{x}}= \\
& \mathscr{F}^{-1}\left(\frac{4 \pi}{3} d^{2}\left(3 \cos ^{2}(\widehat{\mathbf{a}, \boldsymbol{\omega}})-1\right) \mathscr{F}\left(|\psi(t, \mathbf{x})|^{2}\right)(\omega)\right)(\mathbf{x}) .
\end{aligned}
$$

The initial data is the Thomas-Fermi approximation. The converged stationary state is given on Figure 14 where we report the isovalues of the solution. We remark that the stationary state has the property of being elongated along the dipolar direction.

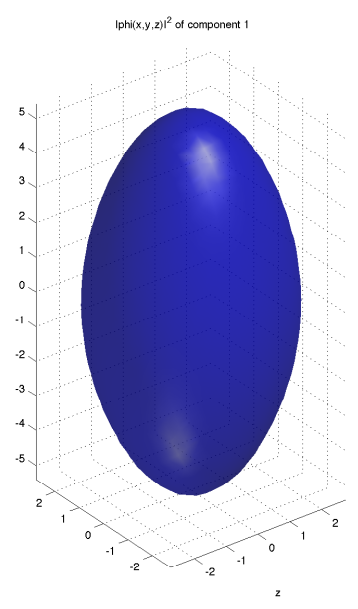

Fig. $1410^{-3}$-isovalues of the modulus for the converged stationary state.

\section{Computation: dynamics}

We develop now the numerical simulation of the dynamics of deterministic (Section 4) or stochastic (Section 5) GPEs (or systems of GPEs) with a rotational term. Let us consider the model equation

$$
\left\{\begin{array}{l}
i \partial_{t} \psi(t, \mathbf{x})=-\frac{1}{2} \Delta \psi(t, \mathbf{x})-\Omega L_{z} \psi(t, \mathbf{x})+V(t, \mathbf{x}) \psi(t, \mathbf{x}) \\
\quad+f\left(|\psi|^{2}\right) \psi(t, \mathbf{x}), \forall t \in \mathbb{R}^{+}, \forall \mathbf{x} \in \mathbb{R}^{d}, \\
\psi(0, \mathbf{x})=\psi_{0}(\mathbf{x}) \in L_{\mathbf{x}}^{2} .
\end{array}\right.
$$

Our aim is to propose some efficient, robust and accurate discretization schemes that reproduce at the discrete level some continuous physical properties (see Section 4.1 . Like for the stationary states computation, we use high-precision pseudo- 
spectral FFT-based discretization schemes. Essentially, we analyze the time-splitting (Section 4.2) and relaxation (Section 4.3) schemes. We discuss some other schemes that we do not recommend (Section 4.4]. We also present a recent idea [27] based on a change of frame for a rotational BEC that should be further investigated in the future since it simplifies the implementation of the standard schemes. We extend the time-splitting and relaxation schemes to multi-components GPEs (Section 4.5. We detail three examples of numerical simulations for a rotating BEC (Section 4.6). The orders of all these schemes are computed and we check the mass and energy conservation properties. The examples are based on GPELab.

Section 5 concerns the extension and study of these numerical schemes for solving the stochastic GPE

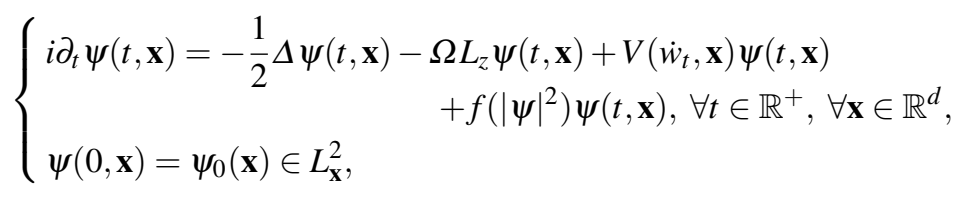

introduced in Section 1.3.3 (page 13).

\subsection{Dynamics of the GPE and continuous/discrete properties}

The dynamics of a BEC is driven by the GPE $(d=1,2,3)$

$$
\left\{\begin{array}{l}
i \partial_{t} \psi(t, \mathbf{x})=-\frac{1}{2} \Delta \psi(t, \mathbf{x})-\Omega L_{z} \psi(t, \mathbf{x})+V(t, \mathbf{x}) \psi(t, \mathbf{x}) \\
\quad+f\left(|\psi|^{2}\right) \psi(t, \mathbf{x}), \forall t \in \mathbb{R}^{+}, \forall \mathbf{x} \in \mathbb{R}^{d}, \\
\psi(0, \mathbf{x})=\psi_{0}(\mathbf{x}) \in L_{\mathbf{x}}^{2} .
\end{array}\right.
$$

We use the notations introduced in Section 2.1. We assume that the initial data is localized in a rectangular domain $\mathscr{O}=]-a_{x}, a_{x}[\times]-a_{y}, a_{y}[\times]-a_{z}, a_{z}\left[\right.$, with $a_{x}, a_{y}$, $a_{z} \in \mathbb{R}^{+}$(depending on the dimension). Let $\Omega=0$ and $V(t, \mathbf{x})=V(\mathbf{x})$. The solution $\psi$ of 90 fulfills some important mathematical/physical properties that the approximation schemes should preserve at the discrete level. In the positive case, the scheme is considered as a "good" scheme. These continuous properties are the following

- Time reversibility: the solution $\psi$ is still the solution of equation 90 after changing the time variable $t \rightarrow-t$ and applying a complex conjugation.

- Dispersion relation: if $V \equiv 0$, the plane wave solution $\psi(t, \mathbf{x})=\rho e^{i(\mathbf{k} \cdot \mathbf{x}-\omega t)}$ satisfies the following dispersion relation

$$
\omega=\frac{|\mathbf{k}|^{2}}{2}+f(|\rho|)
$$

- Gauge transformation: the translation of the potential 


$$
\forall \rho \in \mathbb{R}, \forall \mathbf{x} \in \mathbb{R}^{d}, \quad V(\mathbf{x}) \longrightarrow V(\mathbf{x})+\rho,
$$

creates the following change of phase in the solution

$$
\forall t \in \mathbb{R}^{+}, \forall \mathbf{x} \in \mathbb{R}^{d}, \quad \psi(t, \mathbf{x}) \longrightarrow \psi(t, \mathbf{x}) e^{-i \rho t} .
$$

We remark that the modulus of the solution remains unchanged.

- Mass conservation: the total mass is conserved over the time

$$
\mathscr{N}(\psi)(t):=\|\psi(t, \cdot)\|_{L_{\mathbf{x}}^{2}}^{2}=\int_{\mathbb{R}^{d}}|\psi(t, \mathbf{x})|^{2} d \mathbf{x}=\mathscr{N}\left(\psi_{0}\right), \forall t>0 .
$$

- Energy conservation: if $f\left(|\psi|^{2}\right)=\beta|\psi|^{2}$, the energy is preserved [19]

$$
\mathscr{E}_{0, \beta}(\psi)(t):=\int_{\mathbb{R}^{d}}\left(\frac{1}{2}|\nabla \psi(t, \mathbf{x})|^{2}+V(\mathbf{x})|\psi(t, \mathbf{x})|^{2}+\frac{\beta}{2}|\psi(t, \mathbf{x})|^{4}\right) d \mathbf{x}=\mathscr{E}_{0, \beta}\left(\psi_{0}\right),
$$

for any $t>0$.

We consider the two-dimensional case, the extensions to the dimensions $d=1$ and $d=3$ are direct. Let $\delta t$ be the uniform time step and

$$
\left(\psi_{(j, k)}^{n}\right)_{(j, k) \in \mathscr{F}_{j, k}}
$$

the approximate solution at time $t_{n}=n \delta t$ on a uniform grid $\mathscr{O}_{J, K}$. At the discrete level, the previous properties read

- Time reversibility: changing the indices $(n, n+1) \leftrightarrow(n+1, n)$ lets the solution unchanged: $\psi^{n+1} \leftrightarrow \psi^{n}$.

- Dispersion relation: if $V \equiv 0$ and the initial data is given by

$$
\psi_{(j, k)}^{0}=\rho e^{i \mathbf{k} \cdot \mathbf{x}_{j, k}}
$$

the discrete solution is

$$
\psi_{(j, k)}^{n}=\rho e^{i\left(\mathbf{k} \cdot \mathbf{x}_{j, k}-\omega t_{n}\right)},
$$

where we have the dispersion relation

$$
\omega=\frac{|\mathbf{k}|^{2}}{2}+f(|\rho|) .
$$

This property characterizes the fact that the numerical and exact velocities are the same or not.

- Gauge transformation: the change of potential

$$
\forall \rho \in \mathbb{R}, \forall \mathbf{x} \in \mathbb{R}^{d}, \quad V(\mathbf{x}) \longrightarrow V(\mathbf{x})+\rho,
$$

implies that the solution is modified as follows

$$
\forall n \in \mathbb{N}, \forall(j, k) \in \mathscr{P}_{J, K}, \quad \psi_{(j, k)}^{n} \longrightarrow \psi_{(j, k)}^{n} e^{-i \rho t_{n}},
$$


letting the modulus of the solution unchanged. This characterizes the property that the scheme may induce a phase error in the numerical solution.

- Mass conservation: the discrete mass conservation writes

$$
\forall n \in \mathbb{N}^{*}, \quad \mathscr{N}\left(\psi^{n}\right):=\left\|\psi^{n}\right\|_{\ell_{\pi}^{2}}^{2}=\mathscr{N}\left(\psi^{0}\right),
$$

also stating the $\ell_{\pi}^{2}$-stability of the scheme.

- Energy conservation: if $\Omega=0$ and $f\left(|\psi|^{2}\right)=\beta|\psi|^{2}$, the energy conservation [19] is given by: $\forall n \in \mathbb{N}^{*}, \mathscr{E}_{0, \beta}\left(\psi^{n}\right)=\mathscr{E}_{0, \beta}\left(\psi^{0}\right)$, where

$$
\begin{aligned}
& \mathscr{E}_{0, \beta}\left(\psi^{n}\right):= \\
& \quad\left(h_{x} h_{y}\right)^{1 / 2} \sum_{(j, k) \in \mathscr{P}_{J, K}} \operatorname{Re}\left\{\psi_{j, k}^{n, *}\left(-\frac{1}{2}[[\Delta]] \psi^{n}+[[V]] \psi^{n}+\frac{\beta}{2}\left[\left[\left|\psi^{n}\right|^{4}\right]\right] \psi^{n}\right)_{j, k}\right\} .
\end{aligned}
$$

\subsection{Time-splitting pseudo-spectral schemes for the rotating GPE}

\subsubsection{General principle of time-splitting techniques}

The first schemes that we present is the class of time-splitting schemes for 90 . This scheme, which is known since a long time, has been studied in particular by Strang [112] in a general framework. It has next been applied to the nonlinear Schrödinger equation in [56, 96, 115, 121]. The numerical analysis of the Lie and Strang timesplitting schemes for the Schrödinger equation can be found in particular in [36, 89].

To present the time-splitting schemes (also called fractional step methods), we consider a general dynamical problem. Let $A$ and $B$ be two self-adjoint operators such that: $\mathscr{D}(A) \subset L_{\mathbf{x}}^{2}, \mathscr{D}(B) \subset L_{\mathbf{x}}^{2}$ and $A+B$ a self-adjoint operator on $\mathscr{D}(A) \cap \mathscr{D}(B)$. We denote by $\mathscr{D}(A)$ and $\mathscr{D}(B)$ the domains of the operators $A$ and $B$, respectively. Let us consider the system

$$
\left\{\begin{array}{l}
\partial_{t} \psi(t, \mathbf{x})=A \psi(t, \mathbf{x})+B \psi(t, \mathbf{x}), t \in \mathbb{R}^{+}, \mathbf{x} \in \mathbb{R}^{d} \\
\psi(0, \mathbf{x})=\psi_{0}(\mathbf{x}) \in L_{\mathbf{x}}^{2}
\end{array}\right.
$$

Let $\psi(t, \mathbf{x})=e^{(A+B) t} \psi_{0}(\mathbf{x})$ be the solution of this system, for $t>0$ and $\mathbf{x} \in \mathbb{R}^{d}$. The time-splitting scheme consists in approximating the solution $\psi$ of this problem $v i a$ an approximation of the operator $e^{(A+B) \cdot}$ through the operators $e^{A \cdot}$ and $e^{B \cdot}$. This leads to solve successively two simpler systems. We seek an approximation of the form

$$
\psi(t+\delta t, \mathbf{x})=e^{(A+B) \delta t} \psi(t, \mathbf{x}) \approx e^{a_{1} A \delta t} e^{b_{1} B \delta t} e^{a_{2} A \delta t} e^{b_{2} B \delta t} \ldots e^{a_{p} A \delta t} e^{b_{p} B \delta t} \psi(t, \mathbf{x}),
$$

where $\left\{a_{k}, b_{k}\right\}_{1 \leq k \leq p} \subset \mathbb{R}$ are some computed weights such that the approximation of $e^{(A+B) \delta t}$ has a given order for a local time step $\delta t(\ll 1)$. The two most wellknown time-splitting methods are the Lie (corresponding to $a_{1}=b_{1}=1$ ) and the 
Strang (for $a_{1}=a_{2}=1 / 2, b_{1}=1$ and $b_{2}=0$ ) schemes. They are respectively of order one and two in time. It is possible to get higher-order schemes by suitably choosing the weights [43, 116, 117]. We now focus on the Lie and Strang schemes.

In the case of the GPE with a rotation term, we make the following choice [23, 31]

- we set

$$
A=\frac{i}{2} \Delta+i \Omega L_{z}
$$

which leads to the solution of a linear Schrödinger equation, without potential operator but with a rotational term,

- and

$$
B=-i V(t, \mathbf{x})-i f\left(|\psi(t, \mathbf{x})|^{2}\right),
$$

which gives a nonlinear differential equation that can be solved explicitly in some cases.

The previous decomposition is motivated by the fact that, by using an Alternating Direction Implicit (ADI) method [31], the equation associated to the operator [93] can be solved spectrally by using FFTs. Furthermore, as already mentioned, the equation associated to the operator (94) is solved explicitly. This leads to highly accurate methods. Other choice of operators $A$ and $B$ (e.g. including a part of the potential $V$ in $A$ ) lead to different spectral basis that diagonalize the operators (see [26, 28, 29] for Hermite or Laguerre polynomials).

\subsubsection{Lie time-splitting scheme for 90}

Application of the Lie time-splitting scheme and ADI method. The Lie scheme leads to the following approximation of the solution

$$
\psi(t+\delta t, \mathbf{x}) \approx e^{i\left(\frac{1}{2} \Delta+\Omega L_{z}\right) \delta t} e^{-i\left(V(t, \mathbf{x})+f\left(|\psi(t, \mathbf{x})|^{2}\right)\right) \delta t} \psi(t, \mathbf{x}) .
$$

Let us assume that we want to compute the solution $\psi$ on $[0 ; T]$ that is uniformly discretized into $N$ intervals (a non uniform grid can also be used): $T=N \delta t, N \in \mathbb{N}$. Let us set: $t_{n}:=n \delta t, 0 \leq n \leq N$. For an initial condition $\psi^{0}=\psi_{0}$, the scheme writes: for $0 \leq n \leq N-1$,

1) Compute $\psi_{1}$ such that

$$
\left\{\begin{array}{l}
i \partial_{t} \psi_{1}(t, \mathbf{x})=-\frac{1}{2} \Delta \psi_{1}(t, \mathbf{x})-\Omega L_{z} \psi_{1}(t, \mathbf{x}), n \delta t<t \leq(n+1) \delta t, \forall \mathbf{x} \in \mathbb{R}^{d} \\
\psi_{1}\left(t_{n}, \mathbf{x}\right)=\psi^{n}(\mathbf{x}), \forall \mathbf{x} \in \mathbb{R}^{d}
\end{array}\right.
$$

2) Determine $\psi_{2}$ satisfying 


$$
\left\{\begin{array}{c}
i \partial_{t} \psi_{2}(t, \mathbf{x})=V(t, \mathbf{x}) \psi_{2}(t, \mathbf{x})+f\left(\left|\psi_{2}(t, \mathbf{x})\right|^{2}\right) \psi_{2}(t, \mathbf{x}), \\
\quad n \delta t<t \leq(n+1) \delta t, \forall \mathbf{x} \in \mathbb{R}^{d}, \\
\psi_{2}\left(t_{n}, \mathbf{x}\right)=\psi_{1}\left(t_{n+1}, \mathbf{x}\right), \quad \forall \mathbf{x} \in \mathbb{R}^{d} .
\end{array}\right.
$$

If $\psi^{n+1}(\mathbf{x}):=\psi_{2}\left(t_{n+1}, \mathbf{x}\right)$, we have $\psi^{n+1}(\mathbf{x}) \approx \psi\left(t_{n+1}, \mathbf{x}\right)$.

We consider the two-dimensional case to simplify the presentation (but the oneand three-dimensional cases can be easily deduced). The first step (95) of the splitting scheme can be spectrally resolved for $\Omega=0$ since the Laplacian operator is diagonal in the Fourier space. However, when $\Omega>0$, the situation is more complex since the operator $L_{z}=-i\left(x \partial_{y}-y \partial_{x}\right)$ cannot be directly inverted by using FFTs. Indeed, variable coefficients are present in its expression. A solution to this problem has been proposed by Bao et al. [31]. It consists in applying the ADI method to split the derivations with respect to $x$ and $y$ in two successive steps, allowing to use one-directional FFTs. More precisely, the resulting scheme for solving 95 is given by

1.a) Compute $\psi^{(1)}$ solution to

$$
\left\{\begin{aligned}
i \partial_{t} \psi^{(1)}(t, \mathbf{x})=-\frac{1}{2} \partial_{x}^{2} \psi^{(1)}(t, \mathbf{x}) & \\
& \left.\left.-i \Omega y \partial_{x} \psi^{(1)}(t, \mathbf{x}), \forall t \in\right] t_{n}, t_{n+1}\right], \forall \mathbf{x} \in \mathbb{R}^{2}, \\
\psi^{(1)}\left(t_{n}, \mathbf{x}\right)=\psi^{n}(\mathbf{x}), & \forall \mathbf{x} \in \mathbb{R}^{2} .
\end{aligned}\right.
$$

1.b) Determine $\psi^{(2)}$ such that

$$
\left\{\begin{array}{l}
i \partial_{t} \psi^{(2)}(t, \mathbf{x})=-\frac{1}{2} \partial_{y}^{2} \psi^{(2)}(t, \mathbf{x}) \\
\left.\left.\quad+i \Omega x \partial_{y} \psi^{(2)}(t, \mathbf{x}), \forall t \in\right] t_{n}, t_{n+1}\right], \forall \mathbf{x} \in \mathbb{R}^{2}, \\
\psi^{(2)}\left(t_{n}, \mathbf{x}\right)=\psi^{(1)}\left(t_{n+1}, \mathbf{x}\right), \forall \mathbf{x} \in \mathbb{R}^{2} .
\end{array}\right.
$$

We remark that each partial differential operator appearing in the above equations can be diagonalized by FFTs. After this process, one gets an approximation: $\psi_{1}\left(t_{n+1}, \mathbf{x}\right) \approx \psi^{(2)}\left(t_{n+1}, \mathbf{x}\right)$ for the first step 95 , the second step leading to resolve the ODE 96, which is written as: $\forall \mathbf{x} \in \mathbb{R}^{2}$

$$
\left\{\begin{array}{c}
i \partial_{t} \psi^{(3)}(t, \mathbf{x})=V(t, \mathbf{x}) \psi^{(3)}(t, \mathbf{x}) \\
\left.\left.\quad+f\left(\left|\psi^{(3)}(t, \mathbf{x})\right|^{2}\right) \psi^{(3)}(t, \mathbf{x}), \forall t \in\right] t_{n}, t_{n+1}\right] \\
\psi^{(3)}\left(t_{n}, \mathbf{x}\right)=\psi^{(2)}\left(t_{n+1}, \mathbf{x}\right)
\end{array}\right.
$$

This ordinary differential equation is explicitly integrable thanks to the following result [23].

Lemma 1 Let $\psi^{(3)}$ be the solution to 99. Then, we have

$$
\left.\forall t \in] t_{n}, t_{n+1}\right], \forall \mathbf{x} \in \mathbb{R}^{2}, \quad\left|\psi^{(3)}(t, \mathbf{x})\right|=\left|\psi^{(2)}\left(t_{n+1}, \mathbf{x}\right)\right| .
$$

Dmonstration. The proof is direct since we have: $\left.\forall t \in] t_{n}, t_{n+1}\right]$, 


$$
\begin{aligned}
\partial_{t}\left|\psi^{(3)}(t, \mathbf{x})\right|^{2} & =2 \operatorname{Re}\left(\psi^{(3)^{*}}(t, \mathbf{x}) \partial_{t} \psi^{(3)}(t, \mathbf{x})\right) \\
& =-2 \operatorname{Im}\left(V(t, \mathbf{x})\left|\psi^{(3)}(t, \mathbf{x})\right|^{2}\right)-2 \operatorname{Im}\left(f\left(\left|\psi^{(3)}\right|^{2}\right)\left|\psi^{(3)}(t, \mathbf{x})\right|^{2}\right)=0 .
\end{aligned}
$$

We then get the solution to 99

$$
\forall t \in\left[t_{n}, t_{n+1}\right], \quad \psi^{(3)}(t, \mathbf{x})=e^{-i f\left(\left|\psi^{(2)}\left(t_{n+1}, \mathbf{x}\right)\right|^{2}\right)\left(t-t_{n}\right)-i \int_{t_{n}}^{t} V(s, \mathbf{x}) d s} \psi^{(2)}\left(t_{n+1}, \mathbf{x}\right) .
$$

Finally, the Lie scheme with ADI leads to the approximation $\psi^{n+1}(\mathbf{x}) \approx \psi^{(3)}\left(t_{n+1}, \mathbf{x}\right)$.

Let us remark that the above ADI method implies a loss of symmetry of the global scheme. Indeed, we first solve the equation in the $x$-direction via (97) and then in the $y$-direction by using $(98)$. The symmetry can be obtained easily by alternating the directions at each step. For problem (95), we first solve (97)-(98) at time $t_{n}$ and next (98)-(97) at time $t_{n+1}$.

Pseudo-spectral discretization in space. Let us now consider the problem of the spatial discretization. We again assume that the solution remains confined within the computational box: $\mathscr{O}=]-a_{x}, a_{x}[\times]-a_{y}, a_{y}\left[\right.$, with $a_{x}, a_{y}>0$. We impose some periodic boundary conditions on $\partial \mathscr{O}$ and consider a uniform discretization grid $\mathscr{O}_{J, K}$ associated with $\mathscr{O}$. Let us recall that $\mathscr{P}_{J, K}$ designates the set of grid points indices used for the pseudo-spectral discretization

$$
\mathscr{P}_{J, K}=\left\{(j, k) \in \mathbb{N}^{2} ; 1 \leq j \leq J \text { and } 1 \leq k \leq K\right\} .
$$

We consider an approximation of $\psi^{(m)}$ on this grid that we designate by $\varphi^{(m)}, m=1$, 2, 3. Moreover, the approximation of $\psi^{n}$ is denoted by $\varphi^{n}$. As for the stationary case, we use the following pseudo-spectral discretization of a function $\psi$ in the $x$ and $y$-directions on $\mathscr{O}_{J, K}$ and based on the truncated inverse partial Fourier series, $\forall(j, k) \in \mathscr{P}_{J, K}, \forall t \in \mathbb{R}^{+}$, respectively,

$$
\begin{aligned}
& \psi\left(t, x_{j}, y_{k}\right) \approx \varphi\left(t, x_{j}, y_{k}\right)=\frac{1}{J} \sum_{p=-J / 2}^{J / 2-1} \widehat{\varphi_{p}}\left(t, y_{k}\right) e^{i \mu_{p}\left(x_{j}+a_{x}\right)}, \\
& \psi\left(t, x_{j}, y_{k}\right) \approx \varphi\left(t, x_{j}, y_{k}\right)=\frac{1}{K} \sum_{q=-K / 2}^{K / 2-1} \widehat{\varphi_{q}}\left(t, x_{k}\right) e^{i \lambda_{q}\left(y_{k}+a_{y}\right)},
\end{aligned}
$$

where $\widehat{\varphi_{p}}$ and $\widehat{\varphi_{q}}$ are respectively the Fourier coefficients of the function $\varphi$ in the $x$ and $y$-directions, the Fourier multipliers being: $\mu_{p}=\frac{\pi p}{a_{x}}$ and $\lambda_{q}=\frac{\pi q}{a_{y}}$. The functions $\widehat{\varphi_{p}}$ and $\widehat{\varphi_{q}}$ are written as

$$
\begin{aligned}
\widehat{\varphi_{p}}\left(t, y_{k}\right) & =\sum_{j=0}^{J-1} \varphi\left(t, x_{j}, y_{k}\right) e^{-i \mu_{p}\left(x_{j}+a_{x}\right)}, \\
\widehat{\varphi_{q}}\left(t, x_{j}\right) & =\sum_{k=0}^{K-1} \varphi\left(t, x_{j}, y_{k}\right) e^{-i \lambda_{q}\left(y_{k}+a_{y}\right)} .
\end{aligned}
$$

In the $x$-direction of the Fourier space, we have, $1-J / 2 \leq p \leq J / 2$, 


$$
\forall t \in\left[t_{n}, t_{n+1}\right], \forall 1 \leq k \leq K, \quad i \partial_{t} \widehat{\varphi}_{p}^{(1)}\left(t, y_{k}\right)=\left(\frac{1}{2} \mu_{p}^{2}+\Omega y \mu_{p}\right) \widehat{\varphi}_{p}^{(1)}\left(t, y_{k}\right)
$$

Integrating this equation yields

$$
\forall t \in\left[t_{n}, t_{n+1}\right], \forall 1 \leq k \leq K, \quad \widehat{\varphi}_{p}^{(1)}\left(t, y_{k}\right)=e^{-i\left(\frac{1}{2} \mu_{p}^{2}+\Omega y \mu_{p}\right)\left(t-t_{n}\right)} \widehat{\varphi}_{p}^{(1)}\left(t_{n}, y_{k}\right) .
$$

Similarly, 98 leads to: $1-K / 2 \leq q \leq K / 2$,

$$
\forall t \in\left[t_{n}, t_{n+1}\right], \forall 1 \leq j \leq J, \quad \widehat{\varphi}_{q}^{(2)}\left(t, x_{j}\right)=e^{-i\left(\frac{1}{2} \lambda_{q}^{2}-\Omega x \lambda_{q}\right)\left(t-t_{n}\right)} \widehat{\varphi}_{q}^{(2)}\left(t_{n}, x_{j}\right)
$$

Therefore, the first part of the Lie time-splitting scheme, where we first solve (97) and next 98 on $\left[t_{n}, t_{n+1}\right]$, is implemented as: $\forall(j, k) \in \mathscr{P}_{J, K}$,

$$
\begin{aligned}
\varphi^{(1)}\left(t_{n+1}, x_{j}, y_{k}\right) & =\frac{1}{J} \sum_{p=-J / 2}^{J / 2-1} e^{-i\left(\frac{1}{2} \mu_{p}^{2}+\Omega y_{k} \mu_{p}\right)\left(t_{n+1}-t_{n}\right)} \widehat{\varphi_{p}^{n}}\left(y_{k}\right) e^{i \mu_{p}\left(x_{j}+L_{x}\right)}, \\
\varphi^{(2)}\left(t_{n+1}, x_{j}, y_{k}\right) & \\
= & \frac{1}{K} \sum_{q=-K / 2}^{K / 2-1} e^{-i\left(\frac{1}{2} \lambda_{q}^{2}-\Omega x_{j} \lambda_{q}\right)\left(t_{n+1}-t_{n}\right)} \widehat{\varphi}_{q}^{(1)}\left(t_{n+1}, x_{j}\right) e^{i \lambda_{q}\left(y_{k}+L_{y}\right)} .
\end{aligned}
$$

For solving 100 and for a time-dependent potential $V$, we use the Simpson's quadrature rule

$$
\begin{aligned}
\int_{t_{n}}^{t_{n+1}} V\left(s, x_{j}, y_{k}\right) d s \approx \frac{1}{6}( & V\left(t_{n}, x_{j}, y_{k}\right)+6 V\left(t_{n+1 / 2}, x_{j}, y_{k}\right) \\
& \left.+V\left(t_{n+1}, x_{j}, y_{k}\right)\right)\left(t_{n+1}-t_{n}\right):=\tilde{V}_{n}\left(x_{j}, y_{k}\right) \delta t
\end{aligned}
$$

where $t_{n+\frac{1}{2}}=\left(t_{n}+t_{n+1}\right) / 2$ and $(j, k) \in \mathscr{P}_{J, K}$. This leads to

$$
\varphi^{(3)}\left(t_{n+1}, x_{j}, y_{k}\right)=\varphi^{(2)}\left(t_{n+1}, x_{j}, y_{k}\right) e^{-i \delta t\left(f\left(\left|\varphi^{(2)}\left(t_{n+1}, x_{j}, y_{k}\right)\right|^{2}\right)+\tilde{V}_{n}\left(x_{j}, y_{k}\right)\right)} .
$$

The complete scheme $(103)-(104)$ is first-order accurate in time and spectral in space. In the sequel, the Time-Splitting SPectral scheme of order 1-ADI is denoted by TSSP1-ADI.

\subsubsection{Strang time-splitting scheme for (90)}

To improve the time accuracy of the Lie scheme, we now discuss the second-order Strang TSSP scheme. Since the derivation is quite similar to the previous scheme, we do not detail too much its construction. The Strang time-splitting scheme requires three fractional steps while only one is needed for the Lie scheme. We first resolve the operator $A$ on a time step $\delta t / 2$, next $B$ for $\delta t$ and finally $A$ for $\delta t / 2$. This leads to the following approximation 


$$
\psi(t+\delta t, \mathbf{x}) \approx e^{i\left(\frac{1}{2} \Delta+\Omega L_{z}\right) \frac{\delta t}{2}} e^{-i\left(V(t, \mathbf{x})+f\left(|\psi(t, \mathbf{x})|^{2}\right)\right) \delta t} e^{i\left(\frac{1}{2} \Delta+i \Omega L_{z}\right) \frac{\delta t}{2}} \psi(t, \mathbf{x}),
$$

for $t>0$. An alternative solution consists in changing the roles of $A$ and $B$. The Strang time-splitting scheme with ADI is then

1) Compute $\psi^{(1)}$ solution to

$$
\left\{\begin{array}{c}
i \partial_{t} \psi^{(1)}(t, \mathbf{x})=-\frac{1}{2} \partial_{x}^{2} \psi^{(1)}(t, \mathbf{x}) \\
\left.\left.\quad-i \Omega y \partial_{x} \psi^{(1)}(t, \mathbf{x}), \forall t \in\right] t_{n}, t_{n+\frac{1}{2}}\right], \forall \mathbf{x} \in \mathbb{R}^{2}, \\
\psi^{(1)}\left(t_{n}, \mathbf{x}\right)=\psi_{n}(\mathbf{x}), \forall \mathbf{x} \in \mathbb{R}^{2} .
\end{array}\right.
$$

2) Determine $\psi^{(2)}$ solution of the equation

$$
\left\{\begin{array}{c}
i \partial_{t} \psi^{(2)}(t, \mathbf{x})=-\frac{1}{2} \partial_{y}^{2} \psi^{(2)}(t, \mathbf{x}) \\
\left.\left.\quad+i \Omega x \partial_{y} \psi^{(2)}(t, \mathbf{x}), \forall t \in\right] t_{n}, t_{n+\frac{1}{2}}\right], \forall \mathbf{x} \in \mathbb{R}^{2} \\
\psi^{(2)}\left(t_{n}, \mathbf{x}\right)=\psi^{(1)}\left(t_{n+\frac{1}{2}}, \mathbf{x}\right), \forall \mathbf{x} \in \mathbb{R}^{2}
\end{array}\right.
$$

3) Compute $\psi^{(3)}$ such that

$$
\left\{\begin{aligned}
i \partial_{t} \psi^{(3)}(t, \mathbf{x})= & V(t, \mathbf{x}) \psi^{(3)}(t, \mathbf{x}) \\
& \left.\left.+f\left(\left|\psi^{(3)}(t, \mathbf{x})\right|^{2}\right) \psi^{(3)}(t, \mathbf{x}), \forall t \in\right] t_{n}, t_{n+1}\right], \forall \mathbf{x} \in \mathbb{R}^{2} \\
\psi^{(3)}\left(t_{n}, \mathbf{x}\right)= & \psi^{(2)}\left(t_{n+\frac{1}{2}}, \mathbf{x}\right), \forall \mathbf{x} \in \mathbb{R}^{2}
\end{aligned}\right.
$$

4) Obtain $\psi^{(4)}$ solution to

$$
\left\{\begin{array}{c}
i \partial_{t} \psi^{(4)}(t, \mathbf{x})=-\frac{1}{2} \partial_{y}^{2} \psi^{(4)}(t, \mathbf{x}) \\
\left.\left.\quad+i \Omega x \partial_{y} \psi^{(4)}(t, \mathbf{x}), \forall t \in\right] t_{n}, t_{n+1} / 2\right], \forall \mathbf{x} \in \mathbb{R}^{2}, \\
\psi^{(4)}\left(t_{n}, \mathbf{x}\right)=\psi^{(3)}\left(t_{n+1}, \mathbf{x}\right), \forall \mathbf{x} \in \mathbb{R}^{2} .
\end{array}\right.
$$

5) Determine $\psi^{(5)}$ such that

$$
\left\{\begin{array}{c}
i \partial_{t} \psi^{(5)}(t, \mathbf{x})=-\frac{1}{2} \partial_{x}^{2} \psi^{(5)}(t, \mathbf{x}) \\
\left.\left.\quad-i \Omega y \partial_{x} \psi^{(5)}(t, \mathbf{x}), \forall t \in\right] t_{n}, t_{n+1} / 2\right], \forall \mathbf{x} \in \mathbb{R}^{2} \\
\psi^{(5)}\left(t_{n}, \mathbf{x}\right)=\psi^{(4)}\left(t_{n+\frac{1}{2}}, \mathbf{x}\right), \forall \mathbf{x} \in \mathbb{R}^{2}
\end{array}\right.
$$

The last step gives $\psi^{n+1}(\mathbf{x}) \approx \psi^{(5)}\left(t_{n+\frac{1}{2}}, \mathbf{x}\right)$. Like the Lie scheme, we solve 105 , (106), (108) and (109) by using one-directional FFTs. Equation (107) is explicitly integrated. The Strang scheme is second-order in time and spectral in space which makes it very attractive for the deterministic simulations. Extensions to the one- and three-dimensional cases are direct. The total computational cost of both schemes is $O(M \log M)$, with $M:=J, J K, J K L$, in dimensions $d=1,2,3$, respectively, since we use FFTs. The Strang scheme is time reversible, mass preserving, invariant under 
gauge transformation and the dispersive relation holds. However, it is not energy conserving but the scheme is unconditionally stable for the 2-norm [31]. More details can be found in [10, 24, 25]. In the sequel, the scheme (105)-(109] (with FFTs) is called TSSP2-ADI for Time Splitting SPectral scheme of order 2-ADI.

\subsection{The relaxation scheme for the rotating GPE}

Introduced by Besse [35] for nonlinear Schrödinger equations, the relaxation scheme has some analogies with the standard Crank-Nicolson scheme (Section 4.4) but the nonlinearity is relaxed to avoid a fixed point or a Newton-Raphson method. Therefore, the computational cost is strongly reduced while the scheme is simple to implement. For problem [90, the relaxation scheme is

$$
\left\{\begin{aligned}
\frac{\phi^{n+1 / 2}+\phi^{n-1 / 2}}{2}=f\left(\left|\psi^{n}\right|^{2}\right), & \\
i \frac{\psi^{n+1}-\psi^{n}}{\delta t}=\left(-\frac{1}{2} \Delta-\Omega L_{z}\right)\left(\frac{\psi^{n+1}+\psi^{n}}{2}\right)+\frac{V^{n+1} \psi^{n+1}+V^{n} \psi^{n}}{2} & +\phi^{n+1 / 2}\left(\frac{\psi^{n+1}+\psi^{n}}{2}\right),
\end{aligned}\right.
$$

where $\psi^{n}=\psi\left(t_{n}, \mathbf{x}\right)$ and $V^{n}=V\left(t_{n}, \mathbf{x}\right), 0 \leq n \leq N-1$. The initial conditions are: $\psi^{0}(\mathbf{x})=\psi_{0}(\mathbf{x})$ and $\phi^{-1 / 2}(\mathbf{x})=f\left(\left|\psi^{0}(\mathbf{x})\right|^{2}\right)$. The operator $\left(-\Delta-\Omega L_{z}\right)$ is discretized by the highly accurate pseudo-spectral scheme (see page 30 and equations (101)-(102). Under the same notations, the discrete system is

$$
\left\{\begin{array}{l}
\phi^{n+1 / 2}=\mathbf{c}^{\mathrm{Re}, n}, \\
\mathbb{A}^{\mathrm{Re}, n+1} \psi^{n+1}=\mathbf{b}^{\mathrm{Re}, n},
\end{array}\right.
$$

where $\mathbb{A}^{\mathrm{Re}, n+1}, \mathbf{b}^{\mathrm{Re}, n}$ and $\mathbf{c}^{\mathrm{Re}, n}$ are such that

$$
\begin{aligned}
& \mathbb{A}^{\mathrm{Re}, n+1}:=i \frac{[[I]]}{\delta t}+\frac{1}{4}[[\Delta]]+\frac{1}{2} \Omega\left[\left[L_{z}\right]\right]-\frac{1}{2}\left[\left[V^{n+1}\right]\right]-\frac{1}{2}\left[\left[\phi^{n+1 / 2}\right]\right], \\
& \begin{array}{l}
\mathbf{b}^{\mathrm{Re}, n}:=\left(i \frac{[[I]]}{\delta t}-\frac{1}{4}[[\Delta]]-\frac{1}{2} \Omega\left[\left[L_{z}\right]\right]+\frac{1}{2}\left[\left[V^{n}\right]\right]+\frac{1}{2}\left[\left[\phi^{n+1 / 2}\right]\right]\right) \psi^{n}, \\
\mathbf{c}^{\mathrm{Re}, n}:=2 f\left(\left|\psi^{n}\right|^{2}\right)-\phi^{n-1 / 2}
\end{array}
\end{aligned}
$$

The linear system appearing in (111) and depending on $n$ is solved by a Krylov subspace iterative solver (CGS, BiCGStab, GMRES) [12]. The method is called Relaxation SPectral (ReSP) scheme. The discretization is second-order in time and spectrally accurate in space like for the TSSP2-ADI scheme. Moreover, it is time reversible, mass preserving, unconditionally stable and energy preserving (for a cubic nonlinearity, i.e. $\left.f\left(|\psi|^{2}\right)=\beta|\psi|^{2}\right)$. However, it is not invariant under gauge transformation and the dispersive relation does not hold [10, 35]. The computational cost is $O(M \log M)$ since we again use FFTs. 


\subsection{Other schemes: Euler, Crank-Nicolson, Leap-Frog, rotating frame system}

In this section, we give a brief description of other schemes that could be applied to equation 90 . These schemes are not recommended because of some problems that we detail now. We end by presenting a nice idea that can be found in [27] and which considers a change of frame to simplify the implementation of well-adapted schemes.

The forward or backward Euler schemes are simple schemes in the framework of evolution problems. For (90), the forward Euler scheme is given by

$$
i \frac{\psi^{n+1}-\psi^{n}}{\delta t}=\left(-\frac{1}{2} \Delta-\Omega L_{z}+V^{n}+f\left(\left|\psi^{n}\right|^{2}\right)\right) \psi^{n}
$$

where $\psi^{n}=\psi\left(t_{n}, \mathbf{x}\right)$ and $V^{n}=V\left(t_{n}, \mathbf{x}\right), \forall n \in \mathbb{N}$. The spatial discretization can be obtained, for instance, by using the pseudo-spectral FFT-based approximation leading to

$$
\psi^{n+1}=-i \delta t \mathbf{b}_{\operatorname{Exp}}^{\mathrm{Euler}, n}
$$

where $\mathbf{b}_{\operatorname{Exp}}^{\text {Euler } n}$ is such that

$$
\mathbf{b}_{\operatorname{Exp}}^{\text {Euler }, n}:=\left(i \frac{[[I]]}{\delta t}-\frac{1}{2}[[\Delta]]-\Omega\left[\left[L_{z}\right]+\left[\left[V^{n}\right]\right]+\left[\left[f\left(\left|\psi^{n}\right|^{2}\right)\right]\right]\right) \psi^{n} .\right.
$$

Since there is no linear system to solve, the computational effort is low. However, this first-order scheme is well-known to be conditionally stable under a CFL condition and is therefore useless. The backward Euler scheme is

$$
i \frac{\psi^{n+1}-\psi^{n}}{\delta t}=\left(-\frac{1}{2} \Delta-\Omega L_{z}+V^{n+1}+f\left(\left|\psi^{n+1}\right|^{2}\right)\right) \psi^{n+1}
$$

leading to the linear system

$$
\mathbb{A}_{\operatorname{Imp}}^{\text {Euler }, n} \psi=\mathbf{b}_{\operatorname{Imp}}^{\text {Euler }, n}
$$

where $\mathbb{A}_{\operatorname{Imp}}^{\text {Euler, } n}$ and $\mathbf{b}_{\operatorname{Imp}}^{\text {Euler,n}}$ are such that

$$
\begin{aligned}
& \mathbb{A}_{\operatorname{Imp}}^{\text {Euler }, n}:=i \frac{[[I]]}{\delta t}+\frac{1}{2}[[\Delta]]+\Omega\left[\left[L_{z}\right]\right]-\left[\left[V^{n+1}\right]\right]-\left[\left[f\left(\left|\psi^{n+1}\right|^{2}\right)\right]\right], \\
& \mathbf{b}_{\operatorname{Imp}}^{\text {Euler }, n}:=i \frac{[[I]]}{\delta t} \psi^{n} .
\end{aligned}
$$

The system (116) cannot be directly inverted since the nonlinearity is implicit. At each iteration, a fixed point or a Newton-Raphson method is required to resolve the nonlinearity leading to a computationally expensive scheme. Finally, the scheme is only first-order accurate in time.

The implicit Crank-Nicolson scheme [10, 19, 18] is 


$$
\begin{aligned}
i \frac{\psi^{n+1}-\psi^{n}}{\delta t}=\left(-\frac{1}{2} \Delta-\Omega L_{z}+g\left(\psi^{n+1}, \psi^{n}\right)\right) \frac{\psi^{n+1}+\psi^{n}}{2} & \\
& +\frac{1}{2}\left(V^{n+1} \psi^{n+1}+V^{n} \psi^{n}\right),
\end{aligned}
$$

with

$$
g\left(\psi^{n+1}, \psi^{n}\right):=\int_{0}^{1} f\left(\imath\left|\psi^{n+1}\right|^{2}+(1-\imath)\left|\psi^{n}\right|^{2}\right) d \imath .
$$

Even if this scheme is second-order accurate in time, the presence of the nonlinearity makes it computationally expensive.

The semi-implicit Leap-Frog scheme [10, 18, 19, 20] is

$$
i \frac{\psi^{n+1}-\psi^{n-1}}{\delta t}=\left(-\frac{1}{2} \Delta-\Omega L_{z}\right) \frac{\psi^{n+1}+\psi^{n-1}}{2}+\left(V^{n}+f\left(\left|\psi^{n}\right|^{2}\right)\right) \psi^{n} .
$$

For the initialization, we use

$$
i \frac{\psi^{1}-\psi^{0}}{\delta t}=\left(-\frac{1}{2} \Delta-\Omega L_{z}+V^{0}+f\left(\left|\psi^{0}\right|^{2}\right)\right) \psi^{0}
$$

The major disadvantages of this scheme are that it is conditionally stable and it does not satisfy most of the properties from Section 4.1](see [10]).

In Section 1.3 (page 11), we have seen that a change of variables is used for modeling a rotating condensate. This change of frame, with respect to the reference frame in dimensions $d=2$ and 3 , is based on: for $\mathbf{x}=(x, y) \in \mathbb{R}^{2}$ or $\mathbf{x}=(x, y, z) \in \mathbb{R}^{3}$,

$$
\left\{\begin{array}{l}
x^{\prime}=\cos (\Omega t) x+\sin (\Omega t) y \\
y^{\prime}=-\sin (\Omega t) x+\cos (\Omega t) y
\end{array}\right.
$$

where $\Omega \in \mathbb{R}$ is the rotational speed of the condensate. This gives the relation: $\mathbf{x}^{\prime}=$ $\Omega(t) \mathbf{x}$, where, for $d=2$,

$$
\Omega(t)=\left(\begin{array}{cc}
\cos (\Omega t) & \sin (\Omega t) \\
-\sin (\Omega t) & \cos (\Omega t)
\end{array}\right)
$$

and, for $d=3$,

$$
\Omega(t)=\left(\begin{array}{ccc}
\cos (\Omega t) & \sin (\Omega t) & 0 \\
-\sin (\Omega t) & \cos (\Omega t) & 0 \\
0 & 0 & 1
\end{array}\right)
$$

This change of variables makes the rotation operator $L_{z}$ appear. In [27], the authors propose to compute the dynamics of a rotating condensate by considering the coordinates $\Omega(t) \mathbf{x}$ instead of $\mathbf{x}^{\prime}$. By setting $\psi_{\Omega}(t, \mathbf{x}):=\psi(t, \Omega(t) \mathbf{x})$, where $\psi$ satisfies (90), we obtain that $\psi_{\Omega}$ is solution to 


$$
\left\{\begin{array}{l}
i \partial_{t} \psi_{\Omega}(t, \mathbf{x})=-\frac{1}{2} \Delta \psi_{\Omega}(t, \mathbf{x})+V(t, \Omega(t) \mathbf{x}) \psi_{\Omega}(t, \mathbf{x}) \\
\quad+f\left(\left|\psi_{\Omega}\right|^{2}\right) \psi_{\Omega}(t, \mathbf{x}), \forall t \in \mathbb{R}^{+}, \forall \mathbf{x} \in \mathbb{R}^{d}, \\
\psi_{\Omega}(0, \mathbf{x})=\psi_{0}(\mathbf{x}) \in L_{\mathbf{x}}^{2} .
\end{array}\right.
$$

By simply modifying the potential: $V_{\Omega}(t, \mathbf{x}):=V(t, \Omega(t) \mathbf{x})$, we do not need to discretize the rotation operator which greatly simplifies the resolution of the initialvalue problem. For example, ADI is no longer necessary for the time-splitting scheme. This recent approach is very promising for both the dynamics and stationary states computation and should be further studied.

\subsection{The multi-components case}

We now extend the splitting and relaxation schemes to the case of a system of GPEs with $N_{c}$ components

$$
\left\{\begin{aligned}
& i \partial_{t} \Psi(t, \mathbf{x})=-\frac{1}{2} \Delta \Psi(t, \mathbf{x})- \Omega L_{z} \Psi(t, \mathbf{x})+\mathbf{V}(t, \mathbf{x}) \Psi(t, \mathbf{x}) \\
&+\mathbf{f}(\Psi) \Psi(t, \mathbf{x}), \forall t \in \mathbb{R}^{+}, \forall \mathbf{x} \in \mathbb{R}^{d}, \\
& \Psi(0, \mathbf{x})=\Psi_{0}(\mathbf{x}) \in L_{\mathbf{x}}^{2, N_{c}}, \forall \mathbf{x} \in \mathbb{R}^{d}
\end{aligned}\right.
$$

where $L_{\mathbf{x}}^{2, N_{c}}:=\left(L_{\mathbf{x}}^{2}\right)^{N_{c}}$. We refer to Section 2.5 (page 43 for the notations.

\subsubsection{Time-splitting schemes for a system with $N_{c}$ components}

The strategy adopted here is closely related to the one developed for the onecomponent case. We will see that the explicit formula is only valid for a specific form of the nonlinearity, which explains why the method has some limitations. For the sake of simplicity, we only present the Lie TSSP scheme, the extension to the Strang TSSP scheme being direct. The scheme is given by the two following steps

1) Solve the following system with respect to $\Psi^{(1)}$

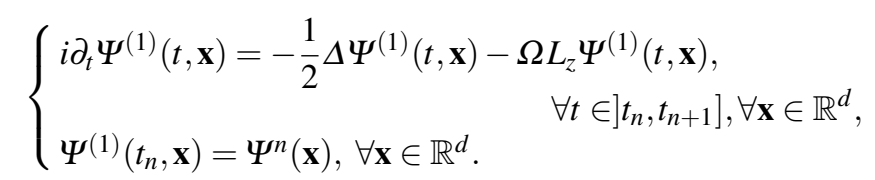

2) Compute $\Psi^{(2)}$ such that

$$
\left\{\begin{array}{c}
i \partial_{t} \Psi^{(2)}(t, \mathbf{x})=\mathbf{V}(t, \mathbf{x}) \Psi^{(2)}(t, \mathbf{x})+\mathbf{f}\left(\Psi^{(2)}(t, \mathbf{x})\right) \Psi^{(2)}(t, \mathbf{x}), \\
\left.\quad \forall t \in] t_{n}, t_{n+1}\right], \forall \mathbf{x} \in \mathbb{R}^{d}, \\
\Psi^{(2)}\left(t_{n}, \mathbf{x}\right)=\Psi^{(1)}\left(t_{n+1}, \mathbf{x}\right), \quad \forall \mathbf{x} \in \mathbb{R}^{d}
\end{array}\right.
$$


Since the operators are diagonal in 121, the unknowns are uncoupled. Therefore, we can apply the ADI method to effectively solve the system of equations by using FFTs. For example, equation $(121)$ is solved in the two-dimensional case through the two following successive steps

$$
\left\{\begin{array}{l}
i \partial_{t} \Psi^{(1,1)}(t, \mathbf{x})=-\frac{1}{2} \partial_{x x} \Psi^{(1,1)}(t, \mathbf{x})-i \Omega y \partial_{x} \Psi^{(1,1)}(t, \mathbf{x}), \\
\Psi^{(1,1)}\left(t_{n}, \mathbf{x}\right)=\Psi^{n}(\mathbf{x}), \quad \forall \mathbf{x} \in \mathbb{R}^{2},
\end{array}\right.
$$

and

$$
\left\{\begin{aligned}
i \partial_{t} \Psi^{(1,2)}(t, \mathbf{x})=-\frac{1}{2} \partial_{y y} \Psi^{(1,2)}(t, \mathbf{x})+ & i \Omega x \partial_{y} \Psi^{(1,2)}(t, \mathbf{x}), \\
& \left.\forall t \in] t_{n}, t_{n+1}\right], \forall \mathbf{x} \in \mathbb{R}^{2}, \\
\Psi^{(1,2)}\left(t_{n}, \mathbf{x}\right)=\Psi^{(1,1)}\left(t_{n+1}, \mathbf{x}\right), & \forall \mathbf{x} \in \mathbb{R}^{2} .
\end{aligned}\right.
$$

For the system of equations (122), we have the following result.

Lemma 2 Let $\Psi^{(2)}$ be the solution of 122. Then, we have

$$
\forall t \in\left[t_{n}, t_{n+1}\right], \quad\left|\Psi^{(2)}(t, \mathbf{x})\right|=\left|\Psi^{(2)}\left(t_{n}, \mathbf{x}\right)\right| .
$$

Dmonstration. First, we have, $\left.\forall t \in] t_{n}, t_{n+1}\right], \forall \mathbf{x} \in \mathbb{R}^{2}$,

$$
\begin{aligned}
\sum_{m=1}^{N_{c}} \partial_{t}\left|\Psi_{m}^{(2)}(t, \mathbf{x})\right|^{2} & =2 \sum_{m=1}^{N_{c}} \operatorname{Re}\left(\Psi_{m}^{(2)}(t, \mathbf{x})^{*} \partial_{t} \Psi_{m}^{(2)}(t, \mathbf{x})\right) \\
& =-2 \sum_{m, o=1}^{N_{c}} \operatorname{Im}\left(\Psi_{m}^{(2)}(t, \mathbf{x})^{*}\left(\mathbf{V}_{m o}(t, \mathbf{x})+\mathbf{f}_{m o}\left(\Psi^{(2)}\right)\right) \Psi_{o}^{(2)}(t, \mathbf{x})\right)
\end{aligned}
$$

By using: $\mathbf{V}_{m o}(t, \mathbf{x})=\mathbf{V}_{o m}(t, \mathbf{x})$ and $\mathbf{f}_{m o}\left(\Psi^{(2)}(t, \mathbf{x})\right)=\mathbf{f}_{o m}\left(\Psi^{(2)}(t, \mathbf{x})\right)$, it follows that

$$
\begin{aligned}
\sum_{m=1}^{N_{c}} \partial_{t}\left|\Psi_{m}^{(2)}(t, \mathbf{x})\right|^{2}=-2 \sum_{N_{c} \geq o>m \geq 1} \operatorname{Im}\left(\left(\mathbf{V}_{m o}(t, \mathbf{x})+\mathbf{f}_{m o}\left(\Psi^{(2)}(t, \mathbf{x})\right)\right)\right. \\
\left.\left(\Psi_{m}^{(2)}(t, \mathbf{x})^{*} \Psi_{o}^{(2)}(t, \mathbf{x})+\Psi_{o}^{(2)}(t, \mathbf{x})^{*} \Psi_{m}^{(2)}(t, \mathbf{x})\right)\right) \\
-2 \sum_{N_{c}} \operatorname{Im}\left(\left(\mathbf{V}_{m m}(t, \mathbf{x})+\mathbf{f}_{m m}\left(\Psi^{(2)}(t, \mathbf{x})\right)\right)\left|\Psi_{m}^{(2)}(t, \mathbf{x})\right|^{2}\right) \\
=-4 \sum_{N_{c} \geq o>m \geq 1} \operatorname{Im}\left(\left(\mathbf{V}_{m o}(t, \mathbf{x})+\mathbf{f}_{m o}\left(\Psi^{(2)}(t, \mathbf{x})\right)\right) \operatorname{Re}\left(\Psi_{m}^{(2)}(t, \mathbf{x})^{*} \Psi_{o}^{(2)}(t, \mathbf{x})\right)\right)=0 .
\end{aligned}
$$

Thus, we conclude that $\left|\Psi^{(2)}(t, \mathbf{x})\right|=\left|\Psi^{(2)}\left(t_{n}, \mathbf{x}\right)\right|, \forall t \in\left[t_{n}, t_{n+1}\right]$.

According to the above result, the modulus of the solution is time preserved. This implies that we can obtain an explicit formulation of the solution by using an exponential operator assuming that $\mathbf{f}$ is such that: $\mathbf{f}(\Psi)=\mathbf{f}(|\Psi|)=\left(\mathbf{f}_{m, \ell}(|\Psi|)\right)_{m, \ell \in\left\{1, \ldots, N_{c}\right\}}$, and that the potential is time-independent: $\mathbf{V}(t, \mathbf{x}):=\mathbf{V}(\mathbf{x})$. Under these assump- 
tions, the system of equations 122 admits the following solution

$$
\Psi^{(2)}(t, \mathbf{x})=e^{-i \mathbf{f}\left(\left|\Psi^{(1,2)}\left(t_{n+1}, \mathbf{x}\right)\right|\right)\left(t-t_{n}\right)-i \mathbf{V}(\mathbf{x})\left(t-t_{n}\right)} \Psi^{(1,2)}\left(t_{n+1}, \mathbf{x}\right) .
$$

This finally leads to the approximation: $\Psi^{n+1}(\mathbf{x}) \approx \Psi^{(2)}\left(t_{n+1}, \mathbf{x}\right)$.

The form (125) of the solution requires the evaluation of an exponential matrix. Moreover, the Lie and Strang splitting schemes need to be symmetrized as for the one-component case because of the ADI method. In the sequel, we call again these methods TSSP1-ADI and TSSP2-ADI, respectively. The computational cost $O(M \log M)$ is essentially related to the FFTs.

\subsubsection{Relaxation scheme for a system with $N_{c}$ components}

For a system of equations, the relaxation scheme is given by

$$
\left\{\begin{array}{l}
\frac{\Phi^{n+1 / 2}+\Phi^{n-1 / 2}}{2}=\mathbf{f}\left(\Psi^{n}\right)+\mathbf{V}^{n}, \mathbf{x} \in \mathbb{R}^{d} \\
\frac{\Psi^{n+1}-\Psi^{n}}{\delta t}=-i\left(-\frac{1}{2} \Delta-\Omega L_{z}+\Phi^{n+1 / 2}\right) \frac{\Psi^{n+1}+\Psi^{n}}{2}, \mathbf{x} \in \mathbb{R}^{d}
\end{array}\right.
$$

where $\Psi^{n}=\Psi\left(t_{n}, \mathbf{x}\right)$ and $\mathbf{V}^{n}=\mathbf{V}\left(t_{n}, \mathbf{x}\right)$. The initial data are given by $\Psi^{0}(\mathbf{x})=\Psi_{0}(\mathbf{x})$ and $\Phi^{-1 / 2}(\mathbf{x})=\mathbf{f}\left(\Psi^{0}(\mathbf{x})\right)$. By using the pseudo-spectral scheme, we are led to solve

$$
\left\{\begin{array}{l}
\mathbb{M}^{\mathrm{Re}, n+1 / 2}=2\left[\left[\mathbf{f}\left(\Psi^{n}\right)\right]\right]-\mathbb{M}^{\mathrm{Re}, n-1 / 2}, \\
\mathbb{A}^{\mathrm{Re}, n+1} \Psi^{n+1}=\mathbb{B}^{\mathrm{Re}, n} \Psi^{n}
\end{array}\right.
$$

where $\Psi^{n}=\left(\psi_{1}^{n}, \ldots, \psi_{N_{c}}^{n}\right)$ is the unknown in $\mathbb{C}^{M N_{c}}$, with $M:=J K$. The nonlinear operator $\mathbb{M}^{R e, n+1 / 2} \in \mathscr{M}_{M N_{c}}(\mathbb{C})$ corresponding to the relaxation is computed by using the nonlinear operator

$$
\left[\left[\mathbf{f}\left(\Psi^{n}\right)\right]\right]:=\left(\begin{array}{cccc}
{\left[\left[\mathbf{f}_{1,1}\left(\Psi^{n}\right)\right]\right]} & {\left[\left[\mathbf{f}_{1,2}\left(\Psi^{n}\right)\right]\right]} & \cdots & {\left[\left[\mathbf{f}_{1, N_{c}}\left(\Psi^{n}\right)\right]\right]} \\
{\left[\left[\mathbf{f}_{2,1}\left(\Psi^{n}\right)\right]\right]} & {\left[\left[\mathbf{f}_{2,2}\left(\Psi^{n}\right)\right]\right]} & \cdots & {\left[\left[\mathbf{f}_{2, N_{c}}\left(\Psi^{n}\right)\right]\right]} \\
\vdots & \vdots & \ddots & \vdots \\
{\left[\left[\mathbf{f}_{N_{c}, 1}\left(\Psi^{n}\right)\right]\right]} & \left.\left[\mathbf{f}_{N_{c}, 2}\left(\Psi^{n}\right)\right]\right] & \cdots & \left.\left[\mathbf{f}_{N_{c}, N_{c}}\left(\Psi^{n}\right)\right]\right]
\end{array}\right) \in \mathscr{M}_{M N_{c}}(\mathbb{R})
$$

where we set $\left[\left[\mathbf{f}_{\ell, m}\left(\Psi^{n}\right)\right]\right]=\left(\mathbf{f}_{\ell, m}\left(\Psi^{n}\left(\mathbf{x}_{j, k}\right)\right)_{(j, k) \in \mathscr{P}_{J, K}}, 1 \leq \ell, m \leq N_{c}\right.$. Furthermore, we choose $\mathbb{M}^{R e,-1 / 2}=\mathbf{f}\left(\Psi_{0}(\mathbf{x})\right)$. The operator $\mathbb{A}^{\operatorname{Re}, n} \in \mathscr{M}_{M N_{c}}(\mathbb{C})$ is such that

$$
\begin{aligned}
& \mathbb{A}^{\mathrm{Re}, n+1} \Psi=\mathbb{A}_{\mathrm{TF}}^{\mathrm{Re}, n+1} \Psi+\mathbb{A}_{\Delta, \Omega}^{\mathrm{Re}} \Psi, \\
& \mathbb{A}_{\mathrm{TF}}^{\mathrm{Re}, n+1} \Psi:=i \frac{[\mathbf{I}]]}{\delta t} \Psi-\frac{1}{2}\left(\left[\left[\mathbf{V}^{n+1}\right]\right]+\mathbb{M}^{\mathrm{Re}, n+1 / 2}\right) \Psi, \\
& \mathbb{A}_{\Delta, \Omega}^{\mathrm{Re}} \Psi:=\frac{1}{2}\left(\frac{1}{2}[[\Delta]]+\Omega\left[\left[L_{z}\right]\right]\right) \Psi .
\end{aligned}
$$


The operator $\mathbb{A}_{\mathrm{TF}}^{\mathrm{Re}, n+1} \in \mathscr{M}_{M N_{c}}(\mathbb{C})$ is defined through the block-matrices

$$
\begin{aligned}
{[[\mathbf{I}]]:=} & \left(\begin{array}{cccc}
{[[I]]} & 0 & \cdots & 0 \\
0 & {[[I]]} & \cdots & 0 \\
\vdots & \vdots & \ddots & \vdots \\
0 & 0 & \cdots & {[[I]]}
\end{array}\right) \in \mathscr{M}_{M N_{c}}(\mathbb{R}), \\
{\left[\left[\mathbf{V}^{n}\right]\right]:=} & \left(\begin{array}{cccc}
{\left[\left[\mathbf{V}_{1,1}^{n}\right]\right]} & {\left[\left[\mathbf{V}_{1,2}^{n}\right]\right]} & \cdots & {\left[\left[\mathbf{V}_{1, N_{c}}^{n}\right]\right]} \\
{\left[\left[\mathbf{V}_{2,1}^{n}\right]\right]} & {\left[\left[\mathbf{V}_{2,2}^{n}\right]\right]} & \cdots & {\left[\left[\mathbf{V}_{2, N_{c}}^{n}\right]\right]} \\
\vdots & \vdots & \ddots & \vdots \\
{\left[\left[\mathbf{V}_{N_{c}, 1}^{n}\right]\right]} & {\left[\left[\mathbf{V}_{N_{c}, 2}^{n}\right]\right]} & \cdots & {\left[\left[\mathbf{V}_{N_{c}, N_{c}}^{n}\right]\right.}
\end{array}\right) \in \mathscr{M}_{M N_{c}}(\mathbb{R}),
\end{aligned}
$$

where: $\left[\left[\mathbf{V}_{\ell, m}^{n}\right]\right]=\left(\mathbf{V}_{\ell, m}^{n}\left(\mathbf{x}_{j, k}\right)\right)_{(j, k) \in \mathscr{P}_{J, K}} \in \mathscr{M}_{M}(\mathbb{R})$. The diagonal operator $\mathbb{A}_{\Delta, \Omega}^{\mathrm{Re}}$ in 127) is implicitly given by

$$
[[\Delta]] \Psi:=\left(\left[\left[\Delta \Psi_{\ell}\right]\right]\right)_{\ell=1, \ldots, N_{c}} \in \mathbb{C}^{M N_{c}} \quad \text { and } \quad\left[\left[L_{z}\right]\right] \Psi:=\left(\left[\left[L_{z} \Psi_{\ell}\right]\right]\right)_{\ell=1, \ldots, N_{c}} \in \mathbb{C}^{M N_{c}}
$$

Finally, the right-hand side is defined by the operator $\mathbb{B}^{\mathrm{Re}, n}: \mathbb{C}^{M N_{c}} \rightarrow \mathbb{C}^{M N_{c}}$

$$
\begin{aligned}
& \mathbb{B}^{\mathrm{Re}, n} \Psi=\mathbb{B}_{\mathrm{TF}}^{\mathrm{Re}, n} \Psi+\mathbb{B}_{\Delta, \Omega}^{\mathrm{Re}} \Psi \\
& \mathbb{B}_{\mathrm{TF}}^{\mathrm{Re}, n} \Psi:=i \frac{[[\mathbf{I}]]}{\delta t} \Psi+\frac{1}{2} \mathbb{M}^{\mathrm{Re}, n+1 / 2} \Psi \\
& \mathbb{B}_{\Delta, \Omega}^{\mathrm{Re}} \Psi:=\frac{1}{2}\left(-\frac{1}{2}[[\Delta]]-\Omega\left[\left[L_{z}\right]\right]\right) \Psi .
\end{aligned}
$$

The linear system in (126) is solved by a preconditioned Krylov subspace iterative solver [12] at a computational $\operatorname{cost} O(M \log M)$. Unlike the splitting schemes, no assumption is required for the relaxation scheme concerning the nonlinear or potential operators.

\subsection{Numerical study of the TSSP1-ADI, TSSP2-ADI and ReSP schemes for the dynamics of rotating GPES}

\subsubsection{Experiment I: dynamics of a rotating BEC in a harmonic trap}

The first numerical experiment consists in solving

$$
\left\{\begin{array}{l}
i \partial_{t} \psi(t, \mathbf{x})=-\frac{1}{2} \Delta \psi(t, \mathbf{x})-\Omega L_{z} \psi(t, \mathbf{x})+V(\mathbf{x}) \psi(t, \mathbf{x}) \\
\quad+\beta|\psi|^{2} \psi(t, \mathbf{x}), \forall t \in[0, T], \forall \mathbf{x} \in \mathbb{R}^{2}, \\
\psi(0, \mathbf{x})=\psi_{0}(\mathbf{x}) \in L_{\mathbf{x}}^{2},
\end{array}\right.
$$


where $\Omega=0.4$ and $\beta=1000$. We fix the quadratic potential

$$
V(\mathbf{x})=\frac{1}{2}\left(\gamma_{x} x^{2}+\gamma_{y} y^{2}\right)
$$

with $\gamma_{x}=\gamma_{y}=1$. The initial data $\psi_{0}$ is computed (by using BESP) as the stationary state (see Figure 15) associated with the problem (129) for the quadratic potential with $\gamma_{x}=\gamma_{y}=2$. The modification of the coefficients increases the confinement of the BEC. This creates a contraction without changing its global shape.

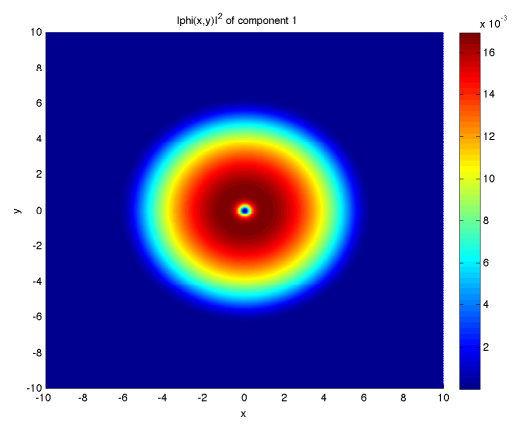

Fig. 15 Initial data $\left|\psi_{0}\right|^{2}$ (on the domain $\left.\mathscr{O}=\right]-10,10\left[{ }^{2}, J=K=2^{9}\right.$ for BESP).

For TSSP1-ADI, TSSP2-ADI and ReSP, we use a time step equal to $\delta t=10^{-3}$ for a final computational time $T=1(T:=N \delta t)$. The pseudo-spectral discretization scheme considers $J=K=2^{9}$ points for the computational domain $\left.\mathscr{O}=\right]-10,10\left[{ }^{2}\right.$. For ReSP, we solve the linear system by using BiCGStab with a stopping criterion set to $\varepsilon^{\text {Krylov }}=10^{-12}$. We report on Figure 16 the solution $\psi^{n \text {,ref }}$ obtained by the ReSP scheme at different times. We remark that the potential confines the condensate. Visualizing the solutions computed by TSSP1-ADI, TSSP2-ADI and ReSP does not allow to make the difference between them.

Let us analyze the spatial accuracy of the schemes. The previous simulation is repeated on different uniform grids $\mathscr{O}_{J, K}$, with $5 \leq J, K \leq 9$, where the reference grid is considered for $J=K=9$. For each grid, the initial data is computed by using the BESP scheme with the parameters of problem $(129)$ for the finest grid. We represent on Figure 17 the maximum error $\operatorname{Err}_{J, K}^{n, \infty}$ between the solution $\psi_{J, K}^{n}$ on the grid $\mathscr{O}_{J, K}$ and the solution $\psi^{n \text {,ref }}$ computed on the grid $\mathscr{O}_{9,9}$ and then extrapolated on the coarser grid $\mathscr{O}_{J, K}$, i.e.: $\operatorname{Err}_{J, K}^{n, \infty}:=\left\|\psi_{J, K}^{n}-\psi^{n \text {,ref }}\right\|_{\infty}$. We also report the error between the energy (without the rotational term) $\mathscr{E}_{0, \beta}\left(\psi_{J, K}^{n}\right)$ on the grid $\mathscr{O}_{J, K}$ and the reference energy $\mathscr{E}_{0, \beta}\left(\psi^{n, \text { ref }}\right): \mathscr{E}_{J, K}^{n, \infty}:=\left|\mathscr{E}_{0, \beta}\left(\psi_{J, K}^{n}\right)-\mathscr{E}_{0, \beta}\left(\psi^{n, \text { ref }}\right)\right|$. We remark that the high accuracy of the TSSP1-ADI, TSSP2-ADI and ReSP is obtained for a sufficiently fine grid, i.e. $J, K \geq 8$. Concerning the coarser grids, the error is relatively important and localized near the central vortex. For this example, the spatial accu- 


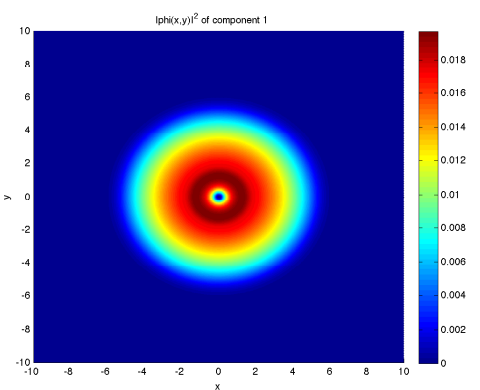

(a) $t=0.13$

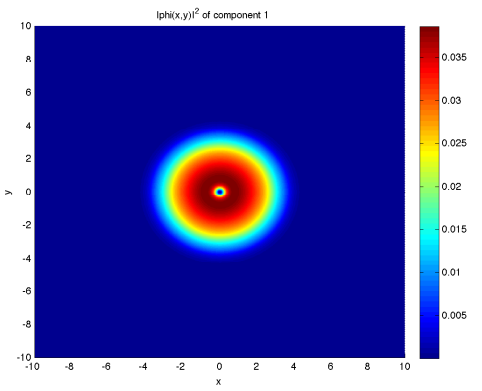

(c) $t=0.39$

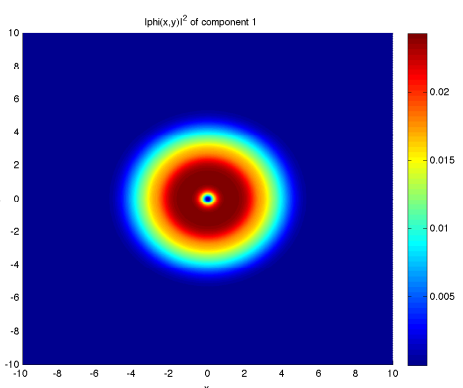

(b) $t=0.26$

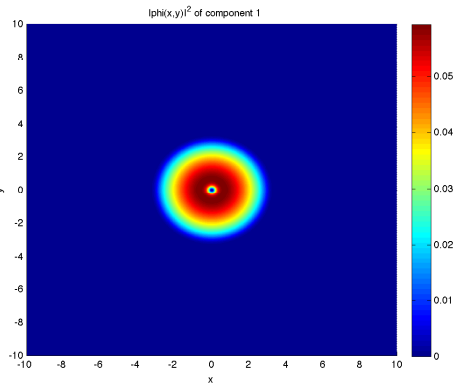

(d) $t=0.52$

Fig. 16 Evolution of the density $|\psi|^{2}$ for problem 129 computed by the ReSP scheme.

racy of the three schemes is about the same. Finally, the energy which is a global quantity is quite accurately computed even for coarse grids.

We are now interested in computing the order of accuracy in time of the three schemes. We also want to analyze the mass and energy (without the rotational term) conservation properties. To numerically obtain the order, we use the Richardson method. Let us denote by $\psi_{\delta t}^{k} \in \mathbb{C}^{M}, k \in \mathbb{N}$, the numerical approximation of a solution $\psi^{k}$ of the problem 1131) at time $t_{k}>0$ by a numerical scheme for a time step $\delta t$. Then, the Richardson method consists in computing the numerical order of accuracy by the expression

$$
p_{k, \text { num }}:=\log _{2}\left(\frac{\left\|\psi_{\delta t}^{k}-\psi_{\delta t / 2}^{k}\right\|_{\ell_{\pi}^{2}}}{\left\|\psi_{\delta t / 2}^{k}-\psi_{\delta t / 4}^{k}\right\|_{\ell_{\pi}^{2}}}\right), 1 \leq k \leq N_{\delta t} .
$$

Indeed, if we assume that the order is $p$, we have

$$
\left\|\psi_{\delta t}^{k}-\psi_{\delta t / 2}^{k}\right\|_{\ell_{\pi}^{2}} \approx C \delta t^{p},
$$

and 


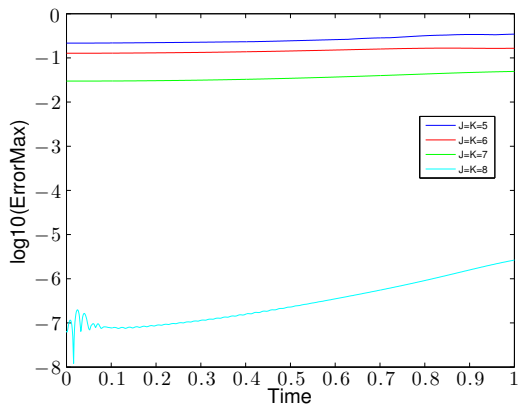

(a) $\operatorname{Err}_{J, K}^{n, \infty}$ for TSSP1-ADI

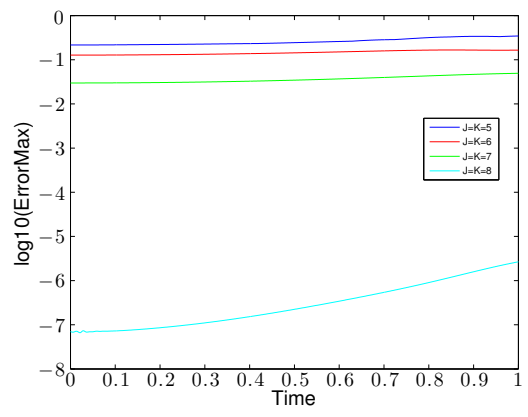

(c) $\operatorname{Err}_{J, K}^{n, \infty}$ for TSSP2-ADI

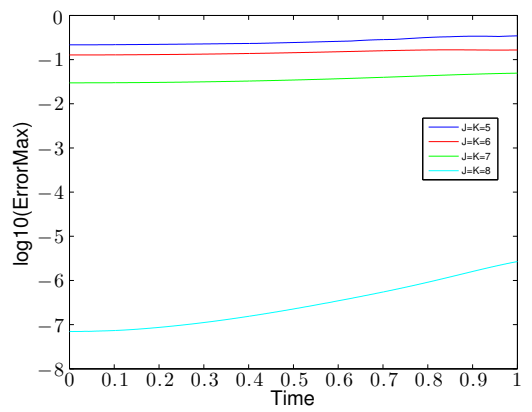

(e) $\operatorname{Err}_{J, K}^{n, \infty}$ for ReSP

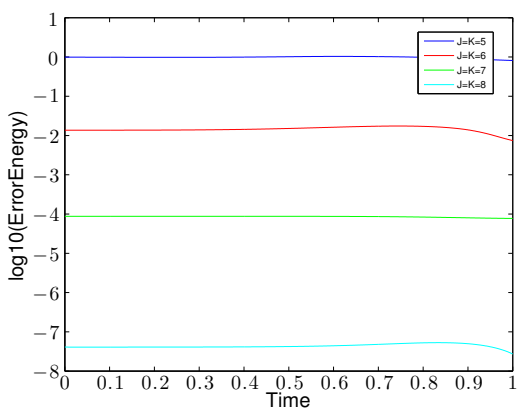

(b) $\mathscr{E}_{J, K}^{n, \infty}$ for TSSP1-ADI

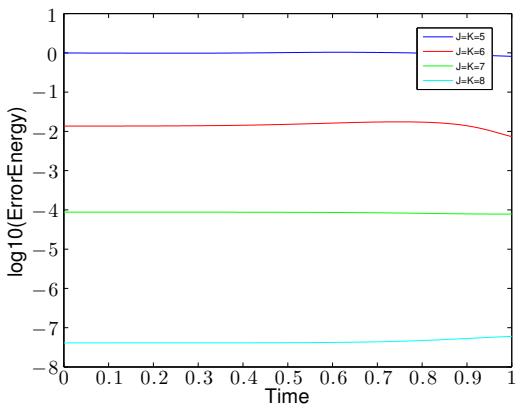

(d) $\mathscr{E}_{J, K}^{n, \infty}$ for TSSP2-ADI

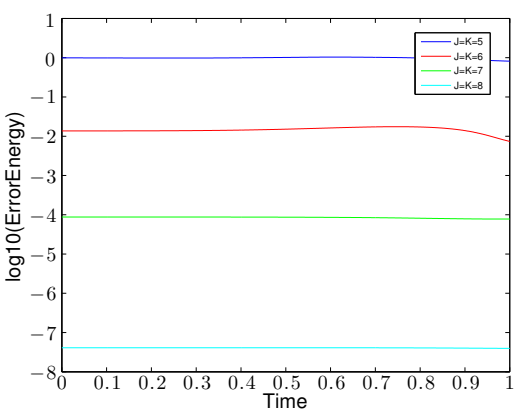

(f) $\mathscr{E}_{J, K}^{n, \infty}$ for ReSP

Fig. 17 Evolution of $\operatorname{Err}_{J, K}^{n, \infty}$ and $\mathscr{E}_{J, K}^{n, \infty}$ for the TSSP1-ADI, TSSP2-ADI and ReSP schemes and different spatial grids.

$$
\left\|\psi_{\delta t / 2}^{k}-\psi_{\delta t / 4}^{k}\right\|_{\ell_{\pi}^{2}} \approx C \frac{\delta t^{p}}{2^{p}},
$$

leading to 130 . Let us consider $J=K=2^{9}$ grid points. We take: $t_{k} N_{\delta t}=k$, with $1 \leq k \leq N_{\delta t}$. We report two cases: $\delta t=10^{-2}$ (Table 5) and $\delta t=10^{-3}$ (Table 6). Here, we introduce the different quantities 


$$
\max p_{N_{\delta t}, \text { num }}:=\max _{1 \leq k \leq N_{\delta t}} p_{k, \text { num }}, \quad \min p_{N_{\delta t}, \text { num }}:=\min _{1 \leq k \leq N_{\delta t}} p_{k, \text { num }}
$$

and

$$
\text { mean } p_{N_{\delta t}, \text { num }}:=\frac{1}{N_{\delta t}} \sum_{k=1}^{N_{\delta t}} p_{k, \text { num }}
$$

For $\delta t=10^{-2}$, the numerical orders of TSSP1-ADI and TSSP2-ADI are in average higher than those expected. This can be explained by the fact that the time step is too large to get a stable scheme and that instabilities arise most particularly because of the rotational term. The ReSP scheme seems to provide a better accuracy. For $\delta t=10^{-3}$, we recover the expected orders for the three schemes.

\begin{tabular}{|c|c|c|c|}
\hline & TSSP1-ADI & TSSP2-ADI & ReSP \\
\hline $\max p_{N_{\delta t}, \text { num }}$ & 5.45 & 6.01 & 2.04 \\
\hline $\min p_{N_{\delta t}}$,num & 1.00 & 2.02 & 1.84 \\
\hline mean $p_{N_{\delta t}, \text { num }}$ & 2.54 & 3.97 & 1.91 \\
\hline
\end{tabular}

Table 5 Numerical orders of the TSSP1-ADI, TSSP2-ADI and ReSP schemes for $\delta t=10^{-2}$.

\begin{tabular}{|c|c|c|c|}
\hline & TSSP1-ADI & TSSP2-ADI & ReSP \\
\hline $\max p_{N_{\delta t}, \text { num }}$ & 1.00 & 2.00 & 1.99 \\
\hline $\min p_{\delta_{\delta t} \text {,num }}$ & 0.99 & 2.00 & 1.99 \\
\hline mean $p_{N_{\delta t}, \text { num }}$ & 1.00 & 2.00 & 1.99 \\
\hline
\end{tabular}

Table 6 Numerical orders of the TSSP1-ADI, TSSP2-ADI and ReSP schemes for $\delta t=10^{-3}$.

We now report (see Figure 18(a) the error on the mass of the solution: $\operatorname{ErrM}^{n}:=$ $\left\|1-\mathscr{N}\left(\psi^{n}\right)\right\|_{\infty}$ and the error (Figure 18(b) on the non rotating energy of the solution: $\mathscr{E}_{0, \beta}\left(\psi^{n}\right)$, for a time step $\delta t=10^{-3}$. We can see that the mass is not exactly conserved but the error is relatively small, even if it increases in time. The ReSP scheme is the scheme that presents the best mass conservation property for this example. In addition, the non rotational energy is well conserved for both the TSSP2-ADI and ReSP schemes while TSSP1-ADI exhibits large fluctuations.

We end the analysis by showing the evolution of the error on the mass (Figure 19(a) and energy without the rotational term $\mathscr{E}_{0, \beta}\left(\psi^{n}\right)$ (Figure 19(b) for $\delta t=10^{-2}$. For the three schemes, we observe that the error on the mass is smaller than when considering the time step $\delta t=10^{-3}$. Nevertheless, the energy also grows substantially in the middle of the simulation for both TSSP1-ADI and TSSP2-ADI. The ReSP scheme conserves correctly the non rotating energy. This example shows that $\mathrm{ReSP}$ is a robust and accurate scheme. 


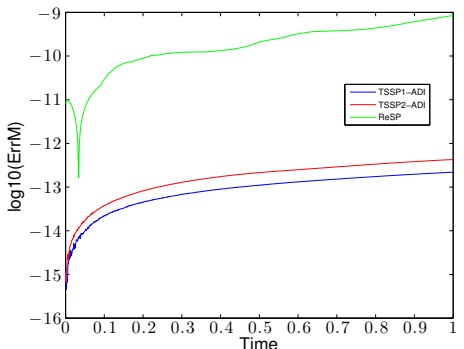

(a) Mass conservation

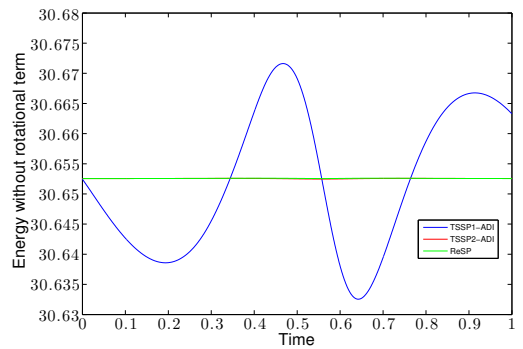

(b) Energy (without rotational term) conservation

Fig. 18 Mass and energy (without rotational term) conservation properties for the three schemes, for $\delta t=10^{-3}$ and problem 131 .

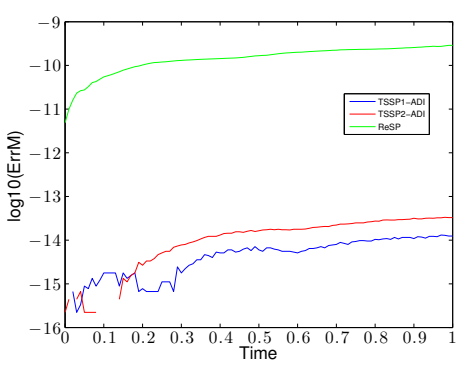

(a) Mass conservation

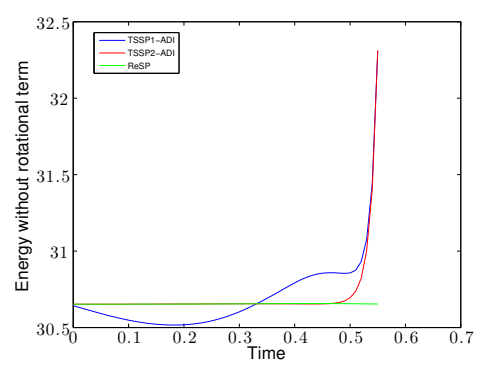

(b) Energy conservation without rotational term

Fig. 19 Mass and energy conservation (without rotational term) properties for the three schemes, for $\delta t=10^{-2}$ and problem 131 .

\subsubsection{Experiment II: dynamics of a BEC in quadratic-plus-quartic trap}

The second example consists in solving the following two-dimensional GPE

$$
\left\{\begin{array}{l}
i \partial_{t} \psi(t, \mathbf{x})=-\frac{1}{2} \Delta \psi(t, \mathbf{x})-\Omega L_{z} \psi(t, \mathbf{x})+V(\mathbf{x}) \psi(t, \mathbf{x}) \\
\quad+\beta|\psi|^{2} \psi(t, \mathbf{x}), \forall t \in[0, T], \forall \mathbf{x} \in \mathbb{R}^{2}, \\
\psi(0, \mathbf{x})=\psi_{0}(\mathbf{x}) \in L_{\mathbf{x}}^{2},
\end{array}\right.
$$

where $\Omega=3.5$ and $\beta=1000$. The potential is the quadratic-plus-quartic potential

$$
V(\mathbf{x})=\frac{1-\alpha}{2}\left(\gamma_{x} x^{2}+\gamma_{y} y^{2}\right)+\frac{\kappa}{4}\left(\gamma_{x} x^{2}+\gamma_{y} y^{2}\right)^{2},
$$

where $\gamma_{x}=\gamma_{y}=1, \alpha=1.2$ and $\kappa=0.7$. To obtain the initial data $\psi_{0}$, we compute the stationary state of (131) for the trapping parameters $\gamma_{x}=\gamma_{y}=1, \alpha=1.2$ and 
$\kappa=0.3$ (see Figure 20). The stationary state is a circular ring with 36 uniformly distributed vortices.

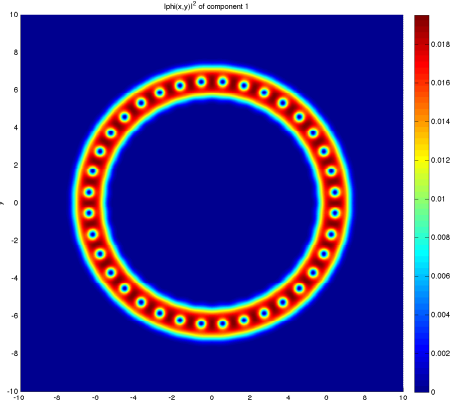

Fig. 20 Density $\left|\psi_{0}\right|^{2}$ of the stationary state (with a domain $\left.\mathscr{O}=\right]-10,10\left[^{2}, J=K=2^{8}\right.$ for BESP).

The parameters for the simulation of the dynamics with TSSP1-ADI, TSSP2ADI and ReSP are: $\delta t=10^{-3}$ for a maximal time of computation $T=1(T:=N \delta t)$, a spatial discretization with $J=K=2^{8}$ points in $\left.\mathscr{O}=\right]-10,10\left[{ }^{2}\right.$. Concerning the ReSP scheme, the linear system is again solved by BiCGStab for a stopping criterion on the residual equal to $\varepsilon^{\mathrm{Krylov}}=10^{-12}$. We report on Figure 21 some snapshots of the solution obtained with TSSP2-ADI. We observe a complex dynamics in the ring BEC. The solutions computed by TSSP2-ADI and ReSP looks the same. Unlike the first experiment, it is not possible here to analyze the spatial accuracy of the schemes since the extrapolated stationary state is not accurate enough on a coarse grid with $J=K=2^{7}$ points. If one considers more grid points ( $J=K \geq 2^{9}$ points), the computational time is too large for GPELab.

We now focus on the numerical order of the TSSP1-ADI, TSSP2-ADI and ReSP schemes and on the discrete mass and energy conservation properties. For $\delta t=$ $10^{-2}$, the orders are not recovered because the time step is too large (Table 7 ). For $\delta t=10^{-3}$ (Table 8), the numerical orders are consistent with their respective theoretical values, meaning that the time step is sufficiently small.

\begin{tabular}{|c|c|c|c|}
\hline & TSSP1-ADI & TSSP2-ADI & ReSP \\
\hline $\max p_{N_{\delta t}}$,num & 1.15 & 1.97 & 1.55 \\
\hline $\min p_{N_{\delta t} \text {, num }}$ & 0.89 & 0.85 & 0.08 \\
\hline mean $p_{N_{\delta t}}$,num & 0.95 & 1.08 & 0.92 \\
\hline
\end{tabular}

Table 7 Numerical orders of the TSSP1-ADI, TSSP2-ADI and ReSP schemes for $\delta t=10^{-2}$.

We consider now the evolution of the error $\operatorname{ErrM}^{n}$ on the mass of the solution (Figure 22(a)) and the non rotational energy $\mathscr{E}_{0, \beta}\left(\psi^{n}\right)$ (Figure 22(b) $)$ for $\delta t=10^{-3}$. 


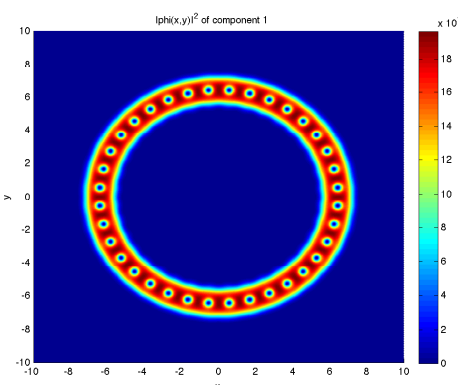

(a) $t=0$

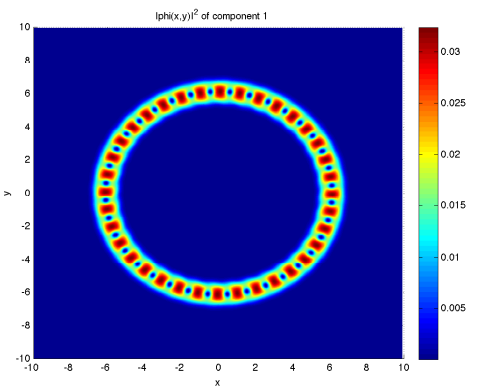

(c) $t=0.14$

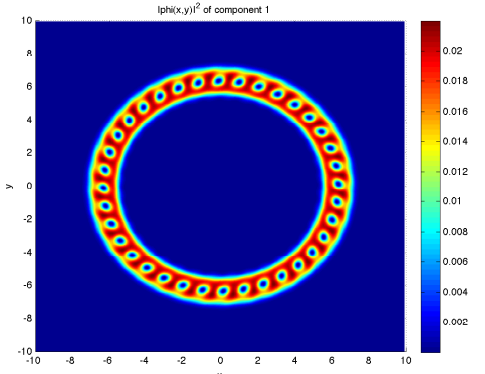

(e) $t=0.28$

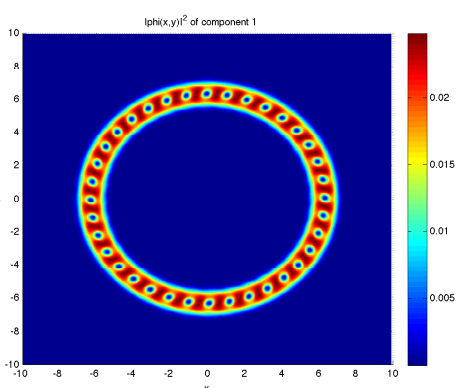

(b) $t=0.07$

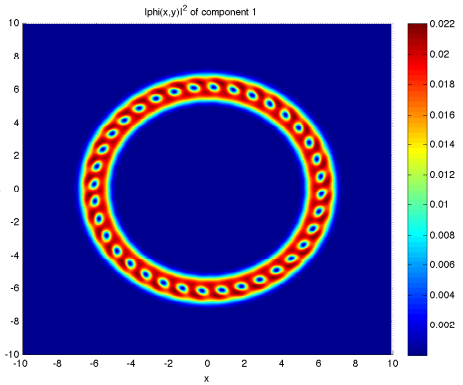

(d) $t=0.21$

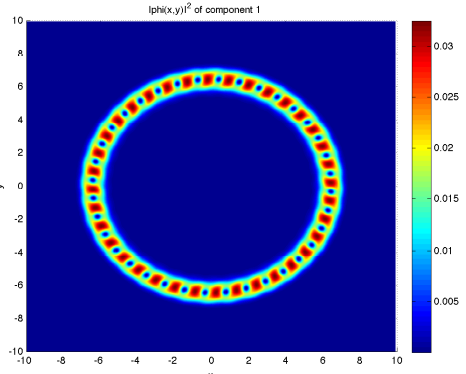

(f) $t=0.35$

Fig. 21 Snapshots of the density $|\psi|^{2}$ computed with TSSP2-ADI for problem 131.

\begin{tabular}{|c|c|c|c|}
\hline & TSSP1-ADI & TSSP2-ADI & ReSP \\
\hline $\max p_{N_{\delta t}, \text { num }}$ & 1.01 & 2.30 & 1.99 \\
\hline $\min p_{N_{\delta t}}$,num & 0.99 & 2.00 & 1.98 \\
\hline mean $p_{N_{\delta t}, \text { num }}$ & 1.00 & 2.02 & 1.99 \\
\hline
\end{tabular}

Table 8 Numerical orders of the TSSP1-ADI, TSSP2-ADI and ReSP schemes for $\delta t=10^{-3}$. 
The mass is not exactly preserved for the three schemes but is numerically acceptable. The energy (without the rotational term) $\mathscr{E}_{0, \beta}\left(\psi^{n}\right)$ is well conserved for the TSSP2-ADI scheme. Concerning the ReSP scheme, the energy fluctuates a little. For the TSSP1-ADI scheme, the energy is not conserved. For $\delta t=10^{-2}$, we have no energy conservation for the three schemes. Globally, TSSP2-ADI is the best scheme for this specific problem.

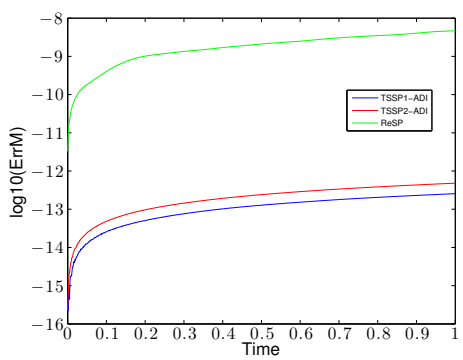

(a) Mass conservation

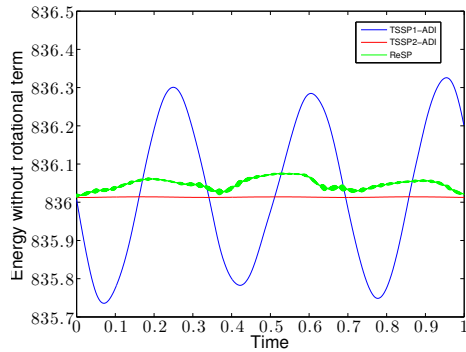

(b) Conservation of the energy without rotational term

Fig. 22 Mass and energy conservation properties for the three schemes, for $\delta t=10^{-3}$ and problem 131.

\subsubsection{Experiment III: dynamics of a $2 \mathrm{~d}$ dark soliton}

The last example consists in the simulation of a black soliton inside a BEC. We consider the two-dimensional GPE

$$
i \partial_{t} \psi(t, \mathbf{x})=\frac{1}{2} \Delta \psi(t, \mathbf{x})+\frac{1}{2}\left(|x|^{2}+|y|^{2}\right) \psi(t, \mathbf{x})+\beta|\psi(t, \mathbf{x})|^{2} \psi(t, \mathbf{x}),
$$

with $\beta=10000$. To get a physically admissible initial data, we first compute a stationary state of 132 by using the BESP scheme for $\delta t=10^{-1}$ and the stopping criterion $\varepsilon=10^{-8}$. The computational domain $\left.\mathscr{O}=\right]-10,10\left[{ }^{2}\right.$ is discretized by a uniform grid with $2^{9}+1$ points in the $x$ - and $y$-directions. We choose the ThomasFermi approximation to initialize the computation. The converged solution is given on Figure 23

We now phase-imprint the black soliton in the condensate and simulate its dynamics. We use ReSP with a time step $\delta t$ equal to $10^{-3}$. The final time of computation is $T=1.5$. A phase-imprinting method [53] is used to initiate the propagation of a black soliton in the condensate. More precisely, the initial data is multiplied by

$$
\xi(\mathbf{x})=e^{i \frac{\Delta \theta_{0}}{2}\left(1+\tanh \left(\frac{x-x_{0}}{s}\right)\right)},
$$




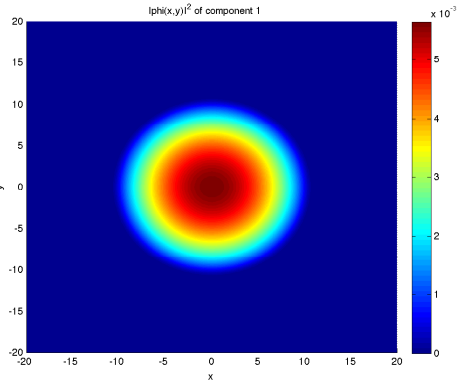

Fig. 23 Modulus of the stationary state computed by BESP with the parameters of Section 4.6.3.

where $\Delta \theta_{0}=\pi / 3, x_{0}=5$ and $s=0.2$. We represent a few snapshots of the computed solution on Figure 24 with the new initial data.

\section{Computation: dynamics \& stochasticity}

The aim of this last Section is to provide a few ideas concerning the extension of the previous schemes when a random term is included into the GPE. Let us consider the stochastic GPE (see Section 1.3.3)

$$
\left\{\begin{array}{l}
i \partial_{t} \psi(t, \mathbf{x})=-\frac{1}{2} \Delta \psi(t, \mathbf{x})-\Omega L_{z} \psi(t, \mathbf{x})+\frac{1}{2}|\mathbf{x}|^{2} \psi(t, \mathbf{x})\left(1+\dot{w}_{t}\right) \\
\quad+\beta|\psi|^{2 \sigma} \psi(t, \mathbf{x}), \forall t \in \mathbb{R}^{+}, \forall \mathbf{x} \in \mathbb{R}^{d}, \\
\psi(0, \mathbf{x})=\psi_{0}(\mathbf{x}) \in L_{\mathbf{x}}^{2},
\end{array}\right.
$$

where $\beta \in \mathbb{R}, \sigma>0$ and $\left(w_{t}\right)_{t \in \mathbb{R}^{+}} \in \mathscr{C}_{t}^{\gamma}\left(\mathbb{R}^{+}\right)$is a Hölder continuous function, with $\gamma \in] 0,1[$. More generally, we consider the following stochastic $\operatorname{GPE}(d=1,2,3)$ for the potential $V\left(\dot{w}_{t}, \mathbf{x}\right):=V(\mathbf{x}) \dot{w}_{t}$

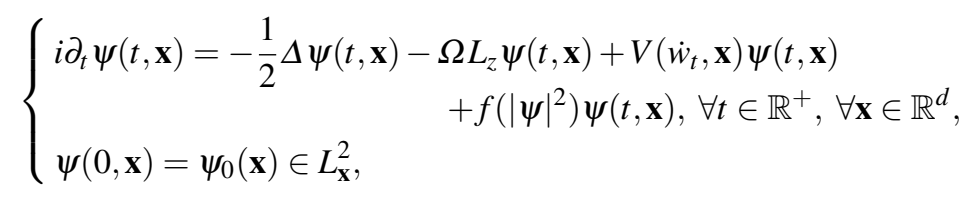

where $f$ is a real-valued polynomial function. 


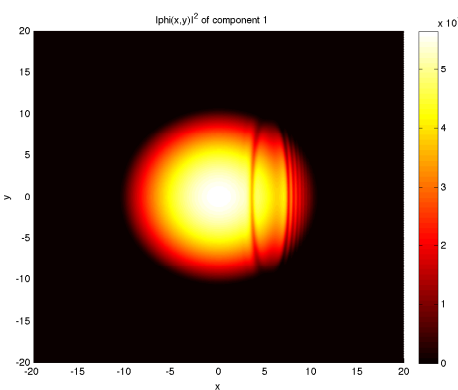

(a) Soliton at time $t=0.25$

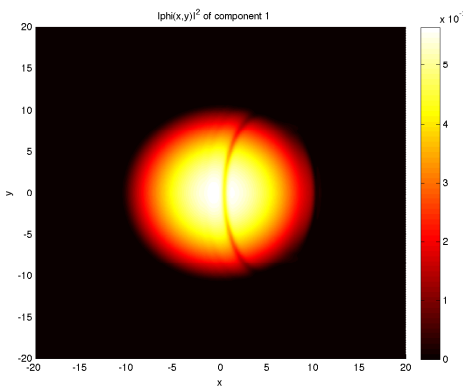

(c) Soliton at time $t=0.75$

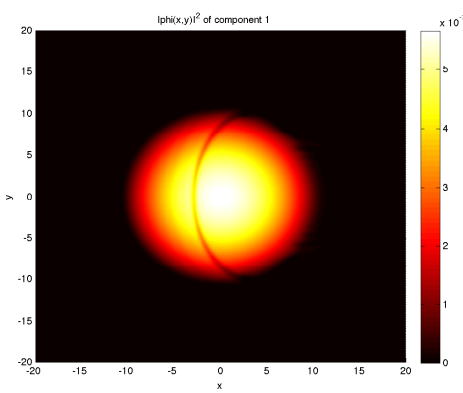

(e) Soliton at time $t=1.25$

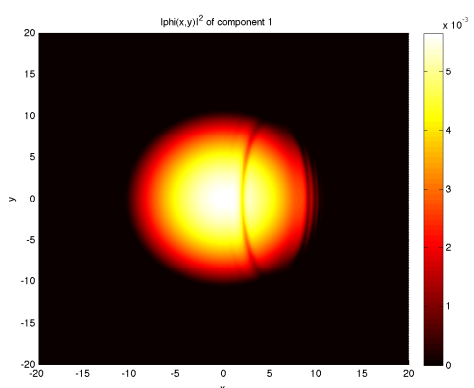

(b) Soliton at time $t=0.5$

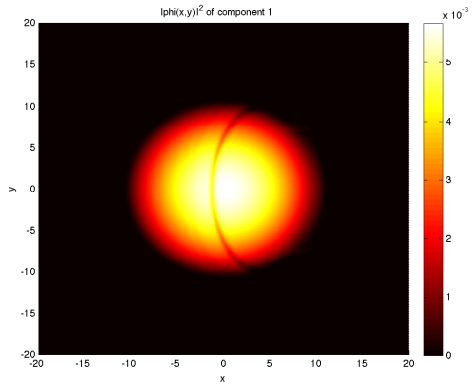

(d) Soliton at time $t=1$

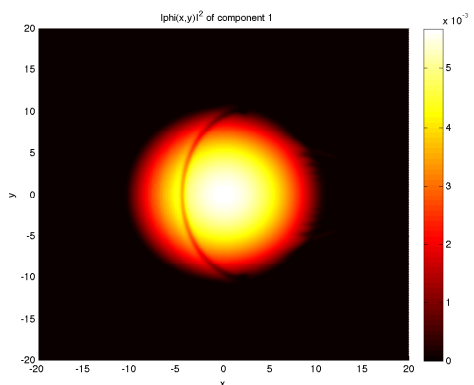

(f) Soliton at time $t=1.5$

Fig. 24 Dynamics of a phase-imprinted black soliton in a BEC by using the ReSP scheme.

\subsection{Numerical schemes for the stochastic GPE}

We discuss the way the stochastic potential has to be discretized in the (Lie and Strang) time-splitting and relaxation schemes. For the time-splitting schemes (Section 5.1.1, the integration is similar to the deterministic case. Concerning the meaning of the time-derivative of a continuous process, we use the definition given 
by Sussmann [114]. For the relaxation scheme (Section 5.1.2), we introduce the Stratonovich product to precise the formal time-derivative of a continuous process that will have to be discretized.

\subsubsection{The time-splitting schemes}

Following Sussmann's approach, we first assume that the process $\left(w_{t}\right)_{t \in \mathbb{R}^{+}}$is in $\mathscr{C}_{t}^{1}\left(\mathbb{R}^{+}\right)$. For the Lie time-splitting scheme, we use the following decomposition of the problem (134): let $\delta t>0, n \in \mathbb{N}$,

1) Solve the system

$$
\left\{\begin{array}{l}
\left.\left.i \partial_{t} \psi_{1}(t, \mathbf{x})=-\frac{1}{2} \Delta \psi_{1}(t, \mathbf{x})-\Omega L_{z} \psi_{1}(t, \mathbf{x}), t \in\right] t_{n}, t_{n+1}\right] \\
\psi_{1}\left(t_{n}, \mathbf{x}\right)=\psi^{n}(\mathbf{x}) .
\end{array}\right.
$$

2) Compute $\psi_{2}$ solution to

$$
\left\{\begin{array}{l}
\left.\left.i \partial_{t} \psi_{2}(t, \mathbf{x})=V\left(\dot{w}_{t}, \mathbf{x}\right) \psi_{2}(t, \mathbf{x})+\beta\left|\psi_{2}(t, \mathbf{x})\right|^{2} \psi_{2}(t, \mathbf{x}), t \in\right] t_{n}, t_{n+1}\right] \\
\psi_{2}\left(t_{n}, \mathbf{x}\right)=\psi_{1}\left(t_{n+1}, \mathbf{x}\right)
\end{array}\right.
$$

The equation 135 is solved by the ADI method and one-directional FFTs like for the deterministic case (see section 4.2.2). For (136), we have seen in Section 4.2.2 that it is possible to exactly integrate the equation for the nonlinearity and potential, and then to obtain an explicit formula. It follows that, for all $t \in\left[t_{n}, t_{n+1}\right]$,

$$
\psi_{2}(t, \mathbf{x})=\psi_{1}\left(t_{n+1}, \mathbf{x}\right) e^{-i f\left(\left|\psi_{1}\left(t_{n+1}, \mathbf{x}\right)\right|^{2}\right)\left(t-t_{n}\right)-i \int_{t_{n}}^{t} V\left(\dot{w}_{s}, \mathbf{x}\right) d s} .
$$

The time integration of the stochastic potential is direct

$$
\int_{t_{n}}^{t} V\left(\dot{w}_{s}, \mathbf{x}\right) d s=\int_{t_{n}}^{t} V(\mathbf{x}) \dot{w}_{s} d s=V(\mathbf{x})\left(w_{t}-w_{t_{n}}\right)=V\left(w_{t}-w_{t_{n}}, \mathbf{x}\right),
$$

leading to the exact formula for 136

$$
\psi_{2}(t, \mathbf{x})=\psi_{1}\left(t_{n+1}, \mathbf{x}\right) e^{-i f\left(\left|\psi_{1}\left(t_{n+1}, \mathbf{x}\right)\right|^{2}\right)\left(t-t_{n}\right)-i V\left(w_{t}-w_{t_{n}}, \mathbf{x}\right)} .
$$

This means that the implementation in the Lie time-splitting scheme is straighforward. Moreover, it is easy to see that this solution is continuous with respect to $\left(w_{t}\right)_{t \in \mathbb{R}^{+}} \in \mathscr{C}_{t}\left(\mathbb{R}^{+}\right)$. Following a similar approach to Sussmann, we can extend the solution to the case of a continuous process by using a density argument. The extension to the Strang time-splitting scheme is trivial. The stochastic schemes are still called TSSP1-ADI and TSSP2-ADI. 


\subsubsection{The relaxation scheme}

In Section 4.3, we derived the relaxation scheme for the deterministic GPE. Concerning the extension to the stochastic case, the main difference is related to the way the noise is discretized. To have a better understanding of how to discretize the derivative of the stochastic process, it is necessary to define the meaning of the following stochastic integral

$$
\int_{t_{n}}^{t_{n+1}} V\left(\dot{w}_{s}, \mathbf{x}\right) \psi(s, \mathbf{x}) d s=V(\mathbf{x}) \int_{t_{n}}^{t_{n+1}} \dot{w}_{s} \psi(s, \mathbf{x}) d s .
$$

Here, we consider this integral as the Stratonovich integral, i.e.

$$
\begin{aligned}
& \int_{t_{n}}^{t_{n+1}} \dot{w}_{s} \psi(s, \mathbf{x}) d s=\int_{t_{n}}^{t_{n+1}} \psi(s, \mathbf{x}) \circ d w_{s}:= \\
& \lim _{\ell \rightarrow \infty} \sum_{\left(s_{k}\right)_{0 \leq k \leq \ell}} \frac{\psi\left(s_{k+1}, \mathbf{x}\right)+\psi\left(s_{k}, \mathbf{x}\right)}{2}\left(w_{s_{k+1}}-w_{s_{k}}\right),
\end{aligned}
$$

where, $\forall \ell \in \mathbb{N},\left(s_{k}\right)_{0 \leq k \leq \ell}$ is a partition of the interval $\left[t_{n}, t_{n+1}\right]$. This type of integral takes its meaning for a Wiener process $\left(W_{t}\right)_{t \in \mathbb{R}^{+}}$(also called the brownian motion) through

$$
\int_{t_{n}}^{t_{n+1}} V\left(\dot{w}_{s}, \mathbf{x}\right) \psi(s, \mathbf{x}) \approx V(\mathbf{x}) \frac{\psi\left(t_{n+1}, \mathbf{x}\right)+\psi\left(t_{n}, \mathbf{x}\right)}{2}\left(w_{t_{n+1}}-w_{t_{n}}\right) .
$$

The associated ReSP scheme related to the discretization 137 for the problem (134) is then

$$
\left\{\begin{array}{l}
\frac{\phi^{n+1 / 2}+\phi^{n-1 / 2}}{2}=f\left(\left|\psi^{n}\right|^{2}\right) \\
i \frac{\psi^{n+1}-\psi^{n}}{\delta t}=\left(-\frac{1}{2} \Delta-\Omega L_{z}+V^{n}+\phi^{n+1 / 2}\right)\left(\frac{\psi^{n+1}+\psi^{n}}{2}\right),
\end{array}\right.
$$

where $\phi^{n+1 / 2}=\phi\left(t_{n+1 / 2}, \mathbf{x}\right), \psi^{n}=\psi\left(t_{n}, \mathbf{x}\right)$ and $V^{n}=V\left(\left(w_{t_{n+1}}-w_{t_{n}}\right) / \delta t, \mathbf{x}\right)$. The initial data are

$$
\psi^{0}(\mathbf{x})=\psi_{0}(\mathbf{x}), \quad \text { and } \quad \phi^{-1 / 2}(\mathbf{x})=\beta\left|\psi_{0}(\mathbf{x})\right|^{2}
$$

\subsection{Numerical examples}

We present here a few numerical simulations. First, we explain how to correctly and efficiently simulate a fractional brownian motion (Section 5.2.1). Next, examples of computations are given for a one-dimensional example. Most particularly, we numerically explore the order of the schemes of the stochastic GPE under consideration (see Section 5.2.2). 


\subsubsection{Simulation of fractional brownian motions}

The simulation of stochastic gaussian processes with stationary increments can be efficiently and accurately realized by using FFTs [123]. Let us recall that a fractional brownian motion (fbm) $\left(W_{t}^{H}\right)_{t \in \mathbb{R}^{+}}$, with Hurst index $\left.H \in\right] 0,1[$, is a gaussian process with the following properties

- $\left(W_{t}^{H}\right)_{t \in \mathbb{R}^{+}}$is continuous and self-similar, i.e.

$$
\forall t \in \mathbb{R}^{+}, \forall a \in \mathbb{R}^{+}, \quad \frac{1}{a^{H}} W_{a t}^{H}=W_{t}^{H} \text { in law, }
$$

- $W_{0}^{H}=0$ almost surely,

- the increments $W_{t}^{H}-W_{s}^{H}$, for all $t, s \in \mathbb{R}^{+}$, such that $t \geq s$, are stationary and follow a normal distribution law with zero mean and $(t-s)^{2 H}$ as variance,

- for all $t, s \in \mathbb{R}^{+}$, such that $t \geq s$, we have

$$
\mathbb{E}\left[W_{t}^{H} W_{s}^{H}\right]=\frac{1}{2}\left(t^{2 H}+s^{2 H}-|t-s|^{2 H}\right) .
$$

Let $\left(t_{j}\right)_{j \in \mathbb{N}}$ be a uniform time discretization of $[0,1]$. Then, we remark that, being given the increments $\left(\delta W_{t_{j+1}}^{H}\right)_{j \in \mathbb{N}}=\left(W_{t_{j+1}}^{H}-W_{t_{j}}^{H}\right)_{j \in \mathbb{N}}$, the fbm can be built through the telescoping sum

$$
W_{t_{j}}^{H}=\sum_{k=1}^{j} \delta W_{t_{k}}^{H}
$$

Therefore, we have to simulate the fbm increments to construct the process. Moreover, thanks to the self-similarity of the process (139), we remark that the simulation of a fbm for $\left(t_{j}\right)_{j \in \mathbb{N}}$ boils down to the simulation of a fbm for the time discretization $\left(t_{j}=j\right)_{j \in \mathbb{N}}$. In this case, for all $k \in \mathbb{Z}$, the autocovariance function $c_{W^{H}}(k)$ of the process $\left(\delta W_{j}^{H}\right)_{j \in \mathbb{N}}$ is given by

$$
c_{W^{H}}(k)=\mathbb{E}\left[\delta W_{j+k}^{H} \delta W_{j}^{H}\right]=\frac{1}{2}\left(|k+1|^{2 H}+|k-1|^{2 H}-2|k|^{2 H}\right) .
$$

If we assume that we want to construct a process of length $N \in \mathbb{N}$ and being given the stationary process $\left(\delta W_{j}^{H}\right)_{j \in\{1, \ldots, N\}}$, its spectral density [54, 123] is such that

$$
\forall j \in\{-N / 2, \ldots, N / 2-1\}, \quad s_{W^{H}}(j)=\sum_{k=-N / 2}^{N / 2-1} c_{W^{H}}(k) e^{-2 \pi i \frac{j k}{N}} .
$$

By using this expression, the following spectral representation of the process [123] can be obtained

$$
\delta W_{k}^{H}=\operatorname{Re}\left(\sqrt{\frac{2}{N}} \sum_{j=-N / 2}^{N / 2-1} \sqrt{s_{W^{H}}(j)} \widehat{W^{1 / 2}}{ }_{j} e^{2 \pi i \frac{j k}{N}}\right)
$$


where $\left(\widehat{W^{1 / 2}}\right)_{j \in\{-N / 2, \ldots, N / 2-1\}}$ is the discrete Fourier transform of the brownian motion. Therefore, the increments of a fbm can be efficiently computed with high precision. We report on Figure 25 the simulation of fbm trajectories for various values of $H$, on a uniformly discretized time interval $[0,1]$ (with $\delta t=10^{-4}$ ).

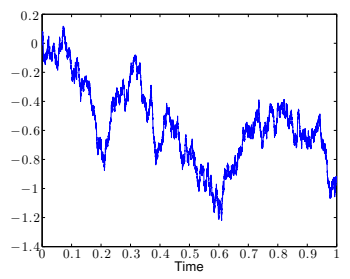

(a) Fractional brownian motion for $H=1 / 2$

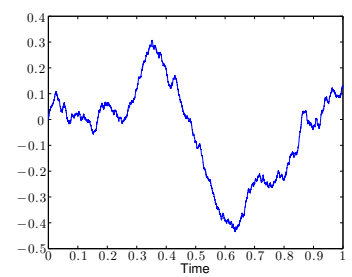

(b) Fractional brownian motion for $H=3 / 4$

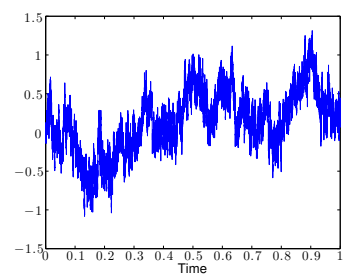

(c) Fractional brownian motion for $H=1 / 4$

Fig. 25 Trajectories of the fbm for various Hurst indices $H$ on the time interval $[0,1]$.

\subsubsection{Order in time of the schemes for the stochastic GPE}

We now numerically study the order in time of the Lie and Strang time-splitting and relaxation schemes for a stochastic potential. The order in time can be understood as the largest real number $p$ such that

$$
\mathbb{E}\left[\left\|\psi_{\delta t}\left(t_{n}, \mathbf{x}\right)-\psi\left(t_{n}, \mathbf{x}\right)\right\|_{L_{\mathbf{x}}^{2}}^{2}\right]^{1 / 2} \leq C(\delta t)^{p}
$$

where $C>0$ is a real-valued positive constant, $\psi$ is the exact solution of the dynamical system and $\psi_{\delta t}$ is the approximation of $\psi$ by using a numerical scheme for a time step $\delta t$. Numerically computing such an order requires the simulation of a large number of trajectories of the process $\left(w_{t}\right)_{t \in \mathbb{R}^{+}}$for the problem 133) since the mean error is approximated by a Monte-Carlo method. For $N(\in \mathbb{N})$ trajectories $\left(w_{t}^{j}\right)_{t \in \mathbb{R}^{+}}, 1 \leq j \leq N$, we compute the numerical approximation of the solution $\psi_{\delta t}^{j}$ of 133 by using one of the numerical schemes. For a sufficiently large value $N$, one gets

$$
\mathbb{E}\left[\left\|\psi_{\delta t}\left(t_{n}, \mathbf{x}\right)-\psi\left(t_{n}, \mathbf{x}\right)\right\|_{L_{\mathbf{x}}^{2}}^{2}\right]^{1 / 2} \approx \sum_{j=1}^{N}\left(\left\|\psi_{\delta t}^{j}\left(t_{n}, \mathbf{x}\right)-\psi\left(t_{n}, \mathbf{x}\right)\right\|_{L_{\mathbf{x}}^{2}}^{2}\right)^{1 / 2} .
$$

Therefore, $p$ can be estimated by a numerical order $p_{\text {num }}$ that is computed thanks to a formula similar to 130

$$
p\left(t_{k}\right) \approx p_{\text {num }}\left(t_{k}\right):=\log _{2}\left(\frac{\mathbb{E}\left[\left\|\psi_{\delta t}\left(t_{k}, \mathbf{x}\right)-\psi_{\delta t / 2}\left(t_{k}, \mathbf{x}\right)\right\|_{L_{\mathbf{x}}^{2}}^{2}\right]^{1 / 2}}{\mathbb{E}\left[\left\|\psi_{\delta t / 2}\left(t_{k}, \mathbf{x}\right)-\psi_{\delta t / 4}\left(t_{k}, \mathbf{x}\right)\right\|_{L_{\mathbf{X}}^{2}}^{2}{ }^{1 / 2}\right.}\right) .
$$


In the following study, we are interested in the fbm of Hurst index $H$ that we denote by $\left.\left(W_{t}^{H}\right)_{t \in \mathbb{R}^{+}}, H \in\right] 0,1[$. As previously mentioned, the fbm are gaussian processes with zero mean that are generalizations of the brownian motion. Moreover, their trajectories are $(H-\eta)$-Hölder continuous, for all $\eta>0$. This means that we can analyze the schemes for processes with various smoothness.

Let us now consider the following one-dimensional stochastic GPE

$$
\left\{\begin{aligned}
i \partial_{t} \psi(t, x)=-\frac{1}{2} \partial_{x}^{2} \psi(t, x)+ & \frac{1}{2} x^{2} \psi(t, x)\left(1+\dot{W}_{t}^{H}\right) \\
& +\beta|\psi|^{2} \psi(t, x), \forall t \in \mathbb{R}^{+}, \forall x \in \mathbb{R}, \\
\psi(0, x)=\psi_{0}(x) \in L_{x}^{2}, &
\end{aligned}\right.
$$

where $\beta=300$ and $\left(W_{t}^{H}\right)_{t \in \mathbb{R}^{+}}$is a fbm with Hurst index $\left.H \in\right] 0,1[$. The initial data $\psi_{0}$ is a stationary state computed by the BESP scheme. The computational domain $\mathscr{O}=]-15,15\left[\right.$ is discretized by a uniform grid $\mathscr{O}_{J}$, where $J=2^{9}$. We fix the time step to $\delta t=10^{-3}$ and the final time of computation to $T=1$. The numerical results of the Monte-Carlo method are based on $N=1000$ trajectories.

We report in Table 9 the numerical orders resulting from formula (141) for the Lie splitting scheme. We remark that the order is linked to the Hurst index $H$. For $H \geq 1 / 2$, the order saturates to 1 while for $H<1 / 2$ it is less than 1 . For $H=1 / 4$, we observe that $N$ is not large enough to yield a good approximation of the order and the scheme seems unstable.

\begin{tabular}{|c|c|c|c|}
\hline & $H=1 / 4$ & $H=1 / 2$ & $H=3 / 4$ \\
\hline $\max _{t_{k}} p_{\text {num }}\left(t_{k}\right)$ & 0.87 & 1.10 & 1.06 \\
\hline $\min _{t_{k}} p_{\text {num }}\left(t_{k}\right)$ & 0.67 & 0.91 & 0.97 \\
\hline $\operatorname{mean}_{t_{k}} p_{\text {num }}\left(t_{k}\right)$ & 0.76 & 1.01 & 1.01 \\
\hline
\end{tabular}

Table 9 Numerical orders of the TSSP1-ADI scheme for the stochastic GPE (142), with various Hurst indices $H$ of the fbm.

We consider now the Strang time-splitting scheme (TSSP2-ADI). The numerical orders are given in Table 10 We observe that the order can be larger than 1 like for $H=3 / 4$. There is no saturation in the numerical order. Furthermore, we notice that the numerical order for a fbm of Hurst index $H=1 / 4$ significantly fluctuates. This is probably due to the fact that $N$ is not large enough to obtain a correct approximation of the order of TSSP2-ADI. The numerical order of the standard brownian motion $(H=1 / 2)$ is not improved in comparison with the TSSP1-ADI scheme.

\begin{tabular}{|c|c|c|c|}
\hline & $H=1 / 4$ & $H=1 / 2$ & $H=3 / 4$ \\
\hline $\max _{t_{k}} p_{\text {num }}\left(t_{k}\right)$ & 1.59 & 1.14 & 1.59 \\
\hline $\min _{t_{k}} p_{\text {num }}\left(t_{k}\right)$ & -0.04 & 0.90 & 0.97 \\
\hline $\operatorname{mean}_{t_{k}} p_{\text {num }}\left(t_{k}\right)$ & 0.89 & 1.01 & 1.33 \\
\hline
\end{tabular}

Table 10 Numerical orders of the TSSP2-ADI scheme for the stochastic GPE 142, with various Hurst indices $H$ for the fbm. 
Table 11 shows the results obtained for the ReSP scheme. For a standard brownian motion $(H=1 / 2)$, the order of the ReSP scheme is lower order than for the time-splitting schemes. In the case of a fbm of Hurst index $H=3 / 4$, the numerical order is larger than 1 and higher than for TSSP2-ADI. The numerical order for the fbm with $H=1 / 4$ is fluctuating. Therefore, it is not possible to conclude on the effective order of the scheme. This is probably due to the fact that the discretization used for the noise (see Equation (137) ) is not adapted to a process with a smoothness lower than the brownian motion.

\begin{tabular}{|c|c|c|c|}
\hline & $H=1 / 4$ & $H=1 / 2$ & $H=3 / 4$ \\
\hline $\max _{t_{k}} p_{\text {num }}\left(t_{k}\right)$ & 1.10 & 0.80 & 1.58 \\
\hline $\min _{t_{k}} p_{\text {num }}\left(t_{k}\right)$ & 0.27 & 0.43 & 1.31 \\
\hline $\operatorname{mean}_{t_{k}} p_{\text {num }}\left(t_{k}\right)$ & 0.84 & 0.58 & 1.49 \\
\hline
\end{tabular}

Table 11 Numerical orders of the ReSP scheme for the stochastic GPE (142), with various Hurst indices $H$ for the $\mathrm{fbm}$ and $\beta=300$.

To complete the numerical simulation for ReSP, we run the same tests as before but with a smaller nonlinearity $\beta$ ( $\beta=100$ here) to show its influence on the order of the scheme. We report the numerical orders on Table 12. For the brownian motion, we observe an improved order of accuracy which is closer to the value 1 obtained for the time-splitting schemes. For the fbm with Hurst index $3 / 4$, the order is practically unchanged. Finally, for $H=1 / 4$, the problem of the discretization remains.

\begin{tabular}{|c|c|c|c|}
\hline & $H=1 / 4$ & $H=1 / 2$ & $H=3 / 4$ \\
\hline $\max _{t_{k}} p_{\text {num }}\left(t_{k}\right)$ & 0.02 & 0.99 & 1.60 \\
\hline $\min _{t_{k}} p_{\text {num }}\left(t_{k}\right)$ & -0.12 & 0.43 & 1.42 \\
\hline $\operatorname{mean}_{t_{k}} p_{\text {num }}\left(t_{k}\right)$ & 0.00 & 0.76 & 1.51 \\
\hline
\end{tabular}

Table 12 Numerical orders of the ReSP scheme for the stochastic GPE (142), with various Hurst indices $H$ for the fbm and $\beta=100$.

As seen before, TSSP2-ADI and ReSP are some suitable schemes for the stochastic GPE (accordingly to $H$ ). When a rotation term is further added, then they should be privileged for a practical computation. Since the related computations are too heavy for GPELab, we do not analyze this problem here. Furthermore, it would be interesting to develop a complete numerical analysis of these schemes to understand the rigorous mathematical properties that can be expected. However, these points are beyond the scope of this paper and can be considered as some open questions.

\section{Conclusion}

In this paper, we have developed some elements related to the modeling and computation of Bose-Einstein Condensates when the Gross-Pitaevskii Equation is used. 
We have introduced a few GPE systems in various physical situations of interest: dynamics, stationary states, multi-components BECs, inclusion of rotation and stochastic terms. Next, we have developed in details some stable pseudo-spectral numerical methods for computing the stationary states of GPEs. A few numerical examples have been produced by using the dedicated Matlab toolbox GPELab. Then, we have explained how to correctly reproduce the dynamics of BECs by using adapted computational schemes (time-splitting and relaxation methods). Again, various numerical examples have been presented to have a better understanding of the schemes. Finally, the extensions of the schemes to a stochastic GPE are explained and numerical simulations based on GPELab show what are the expected properties of the schemes, in particular concerning the accuracy in time.

Acknowledgements This work was partially supported by the French ANR grant ANR-12MONU-0007-02 BECASIM ("Modèles Numériques" call).

\section{References}

1. F. Kh. Abdullaev, B. B. Baizakov, and V. V. Konotop. Dynamics of a Bose-Einstein condensate in optical trap. In Nonlinearity and Disorder: Theory and Applications, volume 45 of NATO Science Series, pages 69-78. Springer Netherlands, 2001.

2. F. Kh. Abdullaev, J. C. Bronski, and R. M. Galimzyanov. Dynamics of a trapped 2d BoseEinstein condensate with periodically and randomly varying atomic scattering length. Physica D: Nonlinear Phenomena, 184(1-4):319 - 332, 2003.

3. F. Kh. Abdullaev, J. C Bronski, and G. Papanicolaou. Soliton perturbations and the random Kepler problem. Physica D: Nonlinear Phenomena, 135(3-4):369 - 386, 2000.

4. S. K. Adhikari. Numerical solution of the two-dimensional Gross-Pitaevskii equation for trapped interacting atoms. Physics Letters A, 265(1):91-96, 2000.

5. A. Aftalion, X. Blanc, and J. Dalibard. Vortex patterns in a fast rotating Bose-Einstein condensate. Physical Review A, 71(2):023611, 2005.

6. A. Aftalion, X. Blanc, and F. Nier. Vortex distribution in the lowest Landau level. Physical Review A, 73(1):011601, 2006.

7. A. Aftalion and Q. Du. Vortices in a rotating Bose-Einstein condensate: Critical angular velocities and energy diagrams in the Thomas-Fermi regime. Physical Review A, 64(6):063603, 2001.

8. A. Aftalion and T. Riviere. Vortex energy and vortex bending for a rotating Bose-Einstein condensate. Physical Review A, 64(4):043611, 2001.

9. M. H. Anderson, J. R. Ensher, M. R. Matthews, C. E. Wieman, and E. A. Cornell. Observation of Bose-Einstein condensation in a dilute atomic vapor. Science, 269(5221):198-201, 1995.

10. X. Antoine, W. Bao, and C. Besse. Computational methods for the dynamics of the nonlinear Schrödinger/Gross-Pitaevskii equations. Computer Physics Communications, 184(12):2621 -2633, 2013.

11. X. Antoine and R. Duboscq. GPELab, a matlab toolbox for computing stationary solutions and dynamics of Gross-Pitaevskii equations. http://gpelab.math.cnrs.fr. 2014.

12. X. Antoine and R. Duboscq. Robust and efficient preconditioned Krylov spectral solvers for computing the ground states of fast rotating and strongly interacting Bose-Einstein condensates. Journal of Computational Physics, 258C:509-523, 2014. 
13. X. Antoine and R. Duboscq. GPELab, a Matlab Toolbox to solve Gross-Pitaevskii Equations I: computation of stationary solutions. Computer Physics Communications, submitted, 2014.

14. V. I. Arnol'd. Mathematical Methods of Classical Mechanics. Graduate Texts in Mathematics. Springer, 1989.

15. A. V. Avdeenkov and K. G. Zloshchastiev. Quantum Bose liquids with logarithmic nonlinearity: Self-sustainability and emergence of spatial extent. Journal of Physics B: Atomic, Molecular and Optical Physics, 44(19):195303, 2011.

16. W. Bao. Ground states and dynamics of multicomponent Bose-Einstein condensates. Multiscale Modeling \& Simulation, 2(2):210-236, 2004.

17. W. Bao and Y. Cai. Ground states of two-component Bose-Einstein condensates with an internal atomic Josephson junction. East Asian J. Appl. Math, 1:49-81, 2011.

18. W. Bao and Y. Cai. Uniform error estimates of finite difference methods for the nonlinear Schrödinger equation with wave operator. SIAM Journal on Numerical Analysis, 50(2):492$521,2012$.

19. W. Bao and Y. Cai. Mathematical theory and numerical methods for Bose-Einstein condensation. Kinetic \& Related Models, 6(1), 2013.

20. W. Bao and Y. Cai. Optimal error estimates of finite difference methods for the GrossPitaevskii equation with angular momentum rotation. Mathematics of Computation, 82(281):99-128, 2013.

21. W. Bao, I. Chern, F. Y. Lim, et al. Efficient and spectrally accurate numerical methods for computing ground and first excited states in Bose-Einstein condensates. Journal of Computational Physics, 219(2):836-854, 2006.

22. W. Bao and Q. Du. Computing the ground state solution of Bose-Einstein condensates by a normalized gradient flow. SIAM Journal on Scientific Computing, 25(5):1674-1697, 2004.

23. W. Bao, D. Jaksch, and P. A. Markowich. Numerical solution of the Gross-Pitaevskii equation for Bose-Einstein condensation. Journal of Computational Physics, 187(1):318-342, 2003.

24. W. Bao, S. Jin, and P. A. Markowich. On time-splitting spectral approximations for the Schrödinger equation in the semiclassical regime. Journal of Computational Physics, 175(2):487-524, 2002.

25. W. Bao, S. Jin, and P. A. Markowich. Numerical study of time-splitting spectral discretizations of nonlinear Schrödinger equations in the semiclassical regimes. SIAM Journal on Scientific Computing, 25(1):27-64, 2003.

26. W. Bao, H. Li, and J. Shen. A generalized-Laguerre-Fourier-Hermite pseudospectral method for computing the dynamics of rotating Bose-Einstein condensates. SIAM Journal on Scientific Computing, 31(5):3685-3711, 2009.

27. W. Bao, D. Marahrens, Q. Tang, and Y. Zhang. A simple and efficient numerical method for computing the dynamics of rotating Bose-Einstein condensates via rotating lagrangian coordinates. SIAM Journal on Scientific Computing, 35(6):A2671-A2695, 2013.

28. W. Bao and J. Shen. A fourth-order time-splitting Laguerre-Hermite pseudospectral method for Bose-Einstein condensates. SIAM Journal on Scientific Computing, 26(6):2010-2028, 2005.

29. W. Bao and J. Shen. A generalized-Laguerre-Hermite pseudospectral method for computing symmetric and central vortex states in Bose-Einstein condensates. Journal of Computational Physics, 227(23):9778-9793, 2008.

30. W. Bao and W. Tang. Ground-state solution of Bose-Einstein condensate by directly minimizing the energy functional. Journal of Computational Physics, 187(1):230-254, 2003.

31. W. Bao and H. Wang. An efficient and spectrally accurate numerical method for computing dynamics of rotating Bose-Einstein condensates. Journal of Computational Physics, 217(2):612-626, 2006.

32. W. Bao, H. Wang, and P. A. Markowich. Ground, symmetric and central vortex states in rotating Bose-Einstein condensates. Communications in Mathematical Sciences, 3(1):57$88,2005$.

33. R. A. Battye, N. R. Cooper, and P. M. Sutcliffe. Stable skyrmions in two-component BoseEinstein condensates. Physical Review Letters, 88:080401, Feb 2002. 
34. D. Baye and J-M. Sparenberg. Resolution of the Gross-Pitaevskii equation with the imaginary-time method on a Lagrange mesh. Physical Review E, 82(5):056701, 2010.

35. C. Besse. A relaxation scheme for the nonlinear Schrödinger equation. SIAM Journal on Numerical Analysis, 42(3):934-952, 2004.

36. C. Besse, B. Bidégaray, and S. Descombes. Order estimates in time of splitting methods for the nonlinear Schrödinger equation. SIAM Journal on Numerical Analysis, 40(1):26-40, 2002.

37. X. Blanc and N. Rougerie. Lowest-Landau-level vortex structure of a Bose-Einstein condensate rotating in a harmonic plus quartic trap. Physical Review A, 77(5):053615, 2008.

38. S. Bose. Planck's law and the light quantum hypothesis. Journal of Astrophysics and Astronomy, 15(1):3-7, 1994.

39. A. S. Bradley and C. W. Gardiner. The stochastic Gross-Pitaevskii equation: III. arXiv preprint cond-mat/0602162, 2006.

40. V. Bretin, S. Stock, Y. Seurin, and J. Dalibard. Fast rotation of a Bose-Einstein condensate. Physical Review Letters, 92(5):050403, 2004.

41. A. J. Brizard. An Introduction to Lagrangian Mechanics. World Scientific Publishing Company Incorporated, 2008.

42. D. A. Butts and D. S. Rokhsar. Predicted signatures of rotating Bose-Einstein condensates. Nature, 397(6717):327-329, 1999.

43. M. Caliari, C. Neuhauser, and M. Thalhammer. High-order time-splitting Hermite and Fourier spectral methods for the Gross-Pitaevskii equation. Journal of Computational Physics, 228(3):822-832, 2009.

44. M. Caliari and S. Rainer. GSGPEs: A matlab code for computing the ground state of systems of Gross-Pitaevskii equations. Computer Physics Communications, 184(3):812 - 823, 2013.

45. E. Cancès. SCF algorithms for HF electronic calculations. In Mathematical Models and Methods for Ab Initio Quantum Chemistry, volume 74 of Lecture Notes in Chemistry, pages 17-43. Springer Berlin Heidelberg, 2000.

46. M. M. Cerimele, M. L. Chiofalo, F. Pistella, S. Succi, and M. P. Tosi. Numerical solution of the Gross-Pitaevskii equation using an explicit finite-difference scheme: An application to trapped Bose-Einstein condensates. Physical Review E, 62(1):1382, 2000.

47. M. L. Chiofalo, S. Succi, and M. P. Tosi. Ground state of trapped interacting Bose-Einstein condensates by an explicit imaginary-time algorithm. Physical Review E, 62(5):7438, 2000.

48. N. R. Cooper, N. K. Wilkin, and J. M. F. Gunn. Quantum phases of vortices in rotating Bose-Einstein condensates. Physical Review Letters, 87:120405, Aug 2001.

49. M. Correggi, N. Rougerie, and J. Yngvason. The transition to a giant vortex phase in a fast rotating Bose-Einstein condensate. Communications in Mathematical Physics, 303(2):451508, 2011.

50. I. Danaila and P. Kazemi. A new Sobolev gradient method for direct minimization of the Gross-Pitaevskii energy with rotation. SIAM Journal on Scientific Computing, 32(5):24472467, 2010

51. K. B. Davis, M-O. Mewes, M. R. van Andrews, N. J. Van Druten, D. S. Durfee, D. M. Kurn, and W. Ketterle. Bose-Einstein condensation in a gas of sodium atoms. Physical Review Letters, 75(22):3969-3973, 1995.

52. L. de Broglie. Ann. Phys., 3, 1925.

53. J. Denschlag, J. E. Simsarian, D. L. Feder, C. W. Clark, L. A. Collins, J. Cubizolles, L. Deng, E. W. Hagley, K. Helmerson, W. P. Reinhardt, et al. Generating solitons by phase engineering of a Bose-Einstein condensate. Science, 287(5450):97-101, 2000.

54. A. B. Dieker and M. Mandjes. On spectral simulation of fractional brownian motion. Probability in the Engineering and Informational Sciences, 17(03):417-434, 2003.

55. C. M. Dion and E. Cancès. Ground state of the time-independent Gross-Pitaevskii equation. Computer Physics Communications, 177(10):787-798, 2007.

56. P. Donnat. Quelques contributions mathématiques en optique non linéaire. $\mathrm{PhD}$ thesis, 1994.

57. M. Edwards and K. Burnett. Numerical solution of the nonlinear Schrödinger equation for small samples of trapped neutral atoms. Physical Review A, 51:1382-1386, 1995. 
58. A. Einstein. Sitzber. Kgl. Preuss. Akad. Wiss., 23:3, 1925.

59. P. Engels, I. Coddington, P. C. Haljan, and E. A. Cornell. Nonequilibrium effects of anisotropic compression applied to vortex lattices in Bose-Einstein condensates. Physical Review Letters, 89(10):100403, 2002.

60. P. Engels, I. Coddington, P. C. Haljan, V. Schweikhard, and E. A. Cornell. Observation of long-lived vortex aggregates in rapidly rotating Bose-Einstein condensates. Physical Review Letters, 90(17): 170405, 2003.

61. R. P. Feynman. Physical Review, 94:262, 1954.

62. R. P. Feynman. Application of quantum mechanics to liquid helium. volume 1 of Progress in Low Temperature Physics, pages 17 - 53. Elsevier, 1955.

63. D. G. Fried, T. C. Killian, L. Willmann, D. Landhuis, S. C. Moss, D. Kleppner, and T. J. Greytak. Bose-Einstein condensation of atomic hydrogen. Physical Review Letters, 81:38113814, Nov 1998.

64. A. Gammal, T. Frederico, and L. Tomio. Improved numerical approach for the time-independent Gross-Pitaevskii nonlinear Schrödinger equation. Physical Review E, 60(2):2421, 1999.

65. C. W. Gardiner, J. R. Anglin, and T. I. A. Fudge. The stochastic Gross-Pitaevskii equation. Journal of Physics B: Atomic, Molecular and Optical Physics, 35(6):1555, 2002.

66. C. W. Gardiner and M. J. Davis. The stochastic Gross-Pitaevskii equation: II. Journal of Physics B: Atomic, Molecular and Optical Physics, 36(23):4731, 2003.

67. J. Garnier, F. Kh. Abdullaev, and B. B. Baizakov. Collapse of a Bose-Einstein condensate induced by fluctuations of the laser intensity. Physical Review A, 69:053607, May 2004.

68. M. E. Gehm, K. M. O'Hara, T. A. Savard, and J. E. Thomas. Dynamics of noise-induced heating in atom traps. Physical Review A, 58:3914-3921, Nov 1998.

69. K. Góral, K. Rzażewski, and T. Pfau. Bose-Einstein condensation with magnetic dipoledipole forces. Physical Review A, 61:051601, Mar 2000.

70. A. Griesmaier, J. Werner, S. Hensler, J. Stuhler, and T. Pfau. Bose-Einstein condensation of chromium. Physical Review Letters, 94:160401, Apr 2005.

71. E. P. Gross. Structure of a quantized vortex in boson systems. Il Nuovo Cimento Series 10, 20(3):454-477, 1961.

72. C. E. Hecht. Physica, 25:262, 1959.

73. W. Heisenberg. The actual content of quantum theoretical kinematics and mechanics. Zhurnal Physik, 43:172-198, 1983.

74. H. F. Hess, G. P. Kochanski, J. M. Doyle, N. Masuhara, D. Kleppner, and T. J. Greytak. Magnetic trapping of spin-polarized atomic hydrogen. Physical Review Letters, 59:672-675, Aug 1987.

75. U. Hohenester. OCTBEC: A Matlab toolbox for optimal quantum control of Bose-Einstein condensates. Computer Physics Communications, 2013.

76. A. D. Jackson, G. M. Kavoulakis, and C. J. Pethick. Solitary waves in clouds of BoseEinstein condensed atoms. Physical Review A, 58:2417-2422, Sep 1998.

77. B. Jackson, J. F. McCann, and C. S. Adams. Vortex formation in dilute inhomogeneous Bose-Einstein condensates. Physical Review Letters, 80:3903-3906, 1998.

78. P. L. Kapitza. Nature, 141:913, 1938.

79. K. Kasamatsu, M. Tsubota, and M. Ueda. Giant hole and circular superflow in a fast rotating Bose-Einstein condensate. Physical Review A, 66:053606, Nov 2002.

80. K. Kasamatsu, M. Tsubota, and M. Ueda. Nonlinear dynamics of vortex lattice formation in a rotating Bose-Einstein condensate. Physical Review A, 67(3):033610, 2003.

81. K. Kasamatsu, M. Tsubota, and M. Ueda. Vortices in multicomponent Bose-Einstein condensates. International Journal of Modern Physics B, 19(11):1835-1904, 2005.

82. M. Koashi and M. Ueda. Exact eigenstates and magnetic response of spin-1 and spin-2 Bose-Einstein condensates. Physical Review Letters, 84:1066-1069, Feb 2000.

83. E. B. Kolomeisky, T. J. Newman, J. P. Straley, and X. Qi. Low-dimensional Bose liquids: beyond the Gross-Pitaevskii approximation. Physical Review Letters, 85(6):1146, 2000.

84. P. Leboeuf and N. Pavloff. Bose-Einstein beams: Coherent propagation through a guide. Physical Review A, 64:033602, Aug 2001. 
85. M. Lewin, P. T. Nam, and N. Rougerie. Derivation of Hartree's theory for generic mean-field Bose systems. Advances in Mathematics, 254(0):570 - 621, 2014.

86. E. H. Lieb and R. Seiringer. Derivation of the Gross-Pitaevskii equation for rotating Bose gases. Communications in mathematical physics, 264(2):505-537, 2006.

87. E. H. Lieb, R. Seiringer, and J. Yngvason. Bosons in a trap: a rigorous derivation of the Gross-Pitaevskii energy functional. In The Stability of Matter: From Atoms to Stars, pages 759-771. Springer, 2005.

88. F. London. Nature, 141:643, 1938.

89. C. Lubich. On splitting methods for Schrödinger-Poisson and cubic nonlinear Schrödinger equations. Mathematics of Computation, 77(264):2141-2153, 2008.

90. E. J. M. Madarassy and V. T. Toth. Numerical simulation code for self-gravitating BoseEinstein condensates. Computer Physics Communications, 184(4):1339 - 1343, 2013.

91. K. W. Madison, F. Chevy, V. Bretin, and J. Dalibard. Stationary states of a rotating BoseEinstein condensate: routes to vortex nucleation. Physical review letters, 86(20):4443-4446, 2001.

92. K. W. Madison, F. Chevy, W. Wohlleben, and J. Dalibard. Vortex formation in a stirred Bose-Einstein condensate. Physical Review Letters, 84(5):806-809, 2000.

93. K. W. Madison, F. Chevy, W. Wohlleben, and J. Dalibard. Vortices in a stirred Bose-Einstein condensate. Journal of Modern Optics, 47(14-15):2715-2723, 2000.

94. H. J. Metcalf and P. Van der Straten. Laser cooling and trapping. Springer, 1999.

95. P. Muruganandam and S. K. Adhikari. Fortran programs for the time-dependent GrossPitaevskii equation in a fully anisotropic trap. Computer Physics Communications, 180(10): 1888 - 1912, 2009.

96. D. Pathria and J. L. 1. Morris. Pseudo-spectral solution of nonlinear Schrödinger equations. Journal of Computational Physics, 87(1):108-125, 1990.

97. C. J. Pethick and H. Smith. Bose-Einstein condensation in dilute gases. Cambridge University Press, 2002.

98. L. P. Pitaevskii. Vortex lines in an imperfect Bose gas. Soviet Physics JETP-USSR, 13(2), 1961.

99. L. P. Pitaevskii and S. Stringari. Bose-Einstein condensation, volume 116. Clarendon press, 2003.

100. S. P. Rath, T. Yefsah, K. J. Günter, M. Cheneau, R. Desbuquois, M. Holzmann, W. Krauth, and J. Dalibard. Equilibrium state of a trapped two-dimensional Bose gas. Physical Review A, 82(1):013609, 2010.

101. N. Rougerie. La théorie de Gross-Pitaevskii pour un condensat de Bose-Einstein en rotation: vortex et transitions de phase. PhD thesis, Université Pierre et Marie Curie-Paris VI, 2010.

102. N. Rougerie. Vortex rings in fast rotating Bose-Einstein condensates. Archive for Rational Mechanics and Analysis, 203(1):69-135, 2012.

103. C. A. Sackett, C. C. Bradley, M. Welling, and R. G. Hulet. Bose-Einstein condensation of lithium. Applied Physics B, 65(4-5):433-440, 1997.

104. L. Salasnich, A. Parola, and L. Reatto. Effective wave equations for the dynamics of cigarshaped and disk-shaped Bose condensates. Physical Review A, 65(4):043614, 2002.

105. T. A. Savard, K. M. O'Hara, and J. E. Thomas. Laser-noise-induced heating in far-off resonance optical traps. Physical Review A, 56:R1095-R1098, Aug 1997.

106. E. Schrdinger. An undulatory theory of the mechanics of atoms and molecules. Phys. Rev., 28:1049-1070, Dec 1926.

107. R. Seiringer. Gross-Pitaevskii theory of the rotating Bose gas. Communications in Mathematical Physics, 229(3):491-509, 2002.

108. T. P. Simula, A. A. Penckwitt, and R. J. Ballagh. Giant vortex lattice deformations in rapidly rotating Bose-Einstein condensates. Physical Review Letters, 92(6):060401, 2004.

109. J. Stenger, S. Inouye, D. M. Stamper-Kurn, H.-J. Miesner, A. P. Chikkatur, and W. Ketterle. Spin domains in ground-state Bose-Einstein condensates. Nature, 396(6709):345-348, 1998.

110. H. T. C. Stoof. Coherent versus incoherent dynamics during Bose-Einstein condensation in atomic gases. Journal of Low Temperature Physics, 114(1-2):11-108, 1999. 
111. H. T. C. Stoof and M. J. Bijlsma. Dynamics of fluctuating Bose-Einstein condensates. Journal of low temperature physics, 124(3-4):431-442, 2001.

112. G. Strang. On the construction and comparison of difference schemes. SIAM Journal on Numerical Analysis, 5(3):506-517, 1968.

113. W. C. Stwalley and L. H. Nosanov. Phy. Rev. Lett., 36:910, 1976.

114. H. J. Sussmann. On the gap between deterministic and stochastic ordinary differential equations. Annals of Probability, 6(1):19-41, 1978.

115. T. R. Taha and M. I. Ablowitz. Analytical and numerical aspects of certain nonlinear evolution equations. ii. numerical, nonlinear Schrödinger equation. Journal of Computational Physics, 55(2):203-230, 1984.

116. M. Thalhammer. High-order exponential operator splitting methods for time-dependent Schrödinger equations. SIAM Journal on Numerical Analysis, 46(4):2022-2038, 2008.

117. M. Thalhammer, M. Caliari, and C. Neuhauser. High-order time-splitting Hermite and Fourier spectral methods. Journal of Computational Physics, 228(3):822-832, 2009.

118. R. P. Tiwari and A. Shukla. A basis-set based fortran program to solve the Gross-Pitaevskii equation for dilute Bose gases in harmonic and anharmonic traps. Computer Physics Communications, 174(12):966 - 982, 2006.

119. M. Tsubota, K. Kasamatsu, and M. Ueda. Vortex lattice formation in a rotating Bose-Einstein condensate. Physical Review A, 65(2):023603, 2002.

120. D. Vudragović, I. Vidanović, A. Balaž, P. Muruganandam, and S. K. Adhikari. C programs for solving the time-dependent Gross-Pitaevskii equation in a fully anisotropic trap. Computer Physics Communications, 183(9):2021 - 2025, 2012.

121. J. A. C. Weideman and B. M. Herbst. Split-step methods for the solution of the nonlinear Schrödinger equation. SIAM Journal on Numerical Analysis, 23(3):485-507, 1986.

122. L. Wen, H. Xiong, and B. Wu. Hidden vortices in a Bose-Einstein condensate in a rotating double-well potential. Physical Review A, 82(5):053627, 2010.

123. Z-M. Yin. New methods for simulation of fractional brownian motion. Journal of Computational Physics, 127(1):66-72, 1996.

124. R. Zeng and Y. Zhang. Efficiently computing vortex lattices in rapid rotating Bose-Einstein condensates. Computer Physics Communications, 180(6):854-860, 2009. 